\title{
Monograph of the Staphylinidae of Crete (Greece). Part I. Diversity and endemism (Insecta: Coleoptera)
}

With 10 figures and 4 tables

\author{
VOLKER ASSING ${ }^{1}$
}

${ }^{1}$ Gabelsbergerstraße 2, 30163 Hannover, Germany. - vassing.hann@t-online.de Published on 2019-12-23

DOI:10.21248/contrib.entomol.69.2.197-238

\begin{abstract}
With a geological history of long isolation, fragmentation, partial submersion, and subsequent rising, characterized by a remarkable diversity in topology, geology, and habitats, and the fifth-largest island of the Mediterranean, Crete has provided an ideal setting for speciation and developing a unique and diverse Staphylinidae fauna. Although beginning already in the middle of the 19th century, the exploration of this fauna gained momentum only recently. Based on a critical revision of literature data, on material collected during several recent field trips, and on additional previously unpublished records, a checklist of the Staphylinidae of Crete is compiled, including as many as 397 named species, with 115 of them reported from the island for the first time and with 48 new species described in the second part of the monograph. Additional unidentified and unnamed species, as well as species doubtfully or erroneously recorded from Crete are listed separately. The systematic, zoogeographic, and ecological composition of the Staphylinidae fauna is characterized with a special focus on the endemics. The latter are represented by 111 named and at least ten unnamed species, account for nearly one-third of the fauna, and mainly belong to the subfamilies Aleocharinae, Scydmaeninae, Pselaphinae, Paederinae, Leptotyphlinae, Staphylininae, and Omaliinae. More than half of them are even locally endemic. The genera with the greatest number of endemic species are Cephennium MüLLER \& Kunze, 1822 of the Scydmaeninae (twelve named species) and Geostiba Thomson, 1858 of the Aleocharinae (nine named species). More than two-thirds of the endemic species are epigeic inhabitants of forests, bush and shrub habitats, grassland, and wetlands, approximately one-fourth is confined to deeper soil strata or endogean, and $8 \%$ are myrmephilous. Slightly more than half ( 59 species; $53 \%$ ) of the endemics are most likely the result of in-situ radiation (18 lineages), the remainder of vicariance with mainland species. A comparison with other Mediterranean islands revealed that (a) total diversity of the Cretan fauna is significantly greater than that of other East Mediterranean islands except Corfu, but much lower than that of Corsica, Sardinia, and Sicily, (b) regarding the number of endemic species Crete ranks third behind Corsica and Sardinia, and (c) the rate of endemism is greater in Crete than in any other Mediterranean island. Explanations for the observed differences in the diversity and composition of the faunas of the islands are discussed. Stichoglossa graeca Bernhauer, 1905, a species of which previously only the lectotype was known, is redescribed and illustrated. Six synonymies are proposed and one name is revalidated: Phloeocharis longipennis Fauvel, 1875 = P. hummleri Bernhauer, 1915, syn. nov.; Phytosus balticus KraAtz, 1859 = P. holtzi Bernhauer, 1935, syn. nov.; Atheta nigra (KrAAtz, 1856) = A. biroi SCHEerpeltz, 1964, syn. nov.; Domene stilicina (ERICHson, $1840)=$ D. lohseiana Bordoni, 1977, syn. nov.; Oedichirus rubronotatus PIC, 1903, revalidated $=$ O. reitteri BERNHAUer, 1908, syn. nov.; Pseudolathra quadricollis (FAuvel, 1875) = P. cretensis Bordoni, 1986, syn. nov. A lectotype is designated for Stichoglossa graeca Bernhauer, 1905. Two species are reported from Greece for the first time.
\end{abstract}




\section{Key words}

Coleoptera, Staphylinidae, Palaearctic region, Mediterranean region, Greece, Crete, diversity, zoogeography, endemism, island biogeography, speciation, taxonomy, new synonymies, revalidation, lectotype designation, new records, redescription, checklist

\section{Zusammenfassung}

Mit ihrer durch lange Isolation sowie durch Zeiten teilweiser Fragmentierung, Submersion und nachfolgender Hebung gekennzeichneten geologischen Vergangenheit, ihrer bemerkenswerten topologischen, geologischen und ökologischen Diversität und ihrer Größe bot die griechische Insel Kreta ideale Voraussetzungen für Speziationsprozesse und die Entwicklung einer einzigartigen und reichhaltigen Staphylinidenfauna. Die Erforschung dieser Fauna begann zwar bereits in der Mitte des 19. Jahrhunderts, ihre gezielte und systematische Erfassung erfolgte jedoch erst in den vergangenen sieben Jahren. Auf der Grundlage einer kritischen Auswertung von Literaturmeldungen, der Ergebnisse mehrerer Forschungsreisen und weiterer bislang unveröffentlichter Nachweise wird eine Checkliste der Staphyliniden erstellt. Insgesamt 397 benannte Arten sind derzeit von Kreta bekannt, von denen 115 erstmals von der Insel nachgewiesen werden; insgesamt 48 Arten, davon 47 endemisch, werden im zweiten Teil der Monografie neu beschrieben. Weitere unidentifizierte und unbenannte Arten sowie irrtümlich oder zweifelhaft von Kreta gemeldete Arten werden separat aufgelistet. Die systematische, zoogeographische und ökologische Zusammensetzung der Staphylinidenfauna Kretas wird charakterisiert. Einen Schwerpunkt bilden dabei die Endemiten, die mit 111 benannten und mindestens zehn unbenannten Arten vertreten sind, insgesamt fast ein Drittel der Gesamtdiversität ausmachen und vor allem zu den Unterfamilien Aleocharinae, Scydmaeninae, Pselaphinae, Paederinae, Leptotyphlinae, Staphylininae und Omaliinae gehören. Mehr als die Hälfte dieser Arten ist auf Kreta lokalendemisch. Die Gattungen mit den meisten Endemiten sind Cephennium Müller \& Kunze, 1822 der Scydmaeninae (zwölf benannte Arten) and Geostiba Thomson, 1858 der Aleocharinae (neun Arten). Bei über zwei Dritteln der Endemiten handelt es sich um epigäische Bewohner von Wald-, Busch-, Strauch-, Gras- und Feuchtbiotopen. Etwa ein Viertel lebt in tieferen Bodenschichten oder ist endogäisch und $8 \%$ sind mit Ameisen assoziiert. Etwas mehr als die Hälfte der Endemiten (59 Arten; 53 \%) bilden nach derzeitigem Kenntnisstand 18 monophyletische Gruppen und sind damit offenbar das Ergebnis von Radiationsprozessen auf der Insel, während sich die übrigen Arten durch Vikarianz mit Arten des Festlands herausgebildet haben. Ein Vergleich mit anderen Mittelmeerinseln ergab, dass (a) die Gesamtdiversität der Fauna Kretas die anderer Inseln der östlichen Mittelmeerraums außer Korfu klar übertrifft, andererseits aber deutlich geringer ist als die Korsikas, Sardiniens und Siziliens, (b) Kreta hinsichtlich der Diversität endemischer Arten nur von Korsika und Sardinien übertroffen wird, und dass (c) der Anteil der Endemiten an der Gesamtdiversität auf Kreta höher ist als auf allen anderen Mittelmeerinseln. Erklärungen für die beobachteten Unterschiede in der Zusammensetzung und Diversität der Inseln werden diskutiert. Stichoglossa graeca BERNHAUER, 1905, von der zuvor nur der Lectotypus bekannt war, wird beschrieben und abgebildet. Sechs Namen werden synonymisiert, einer revalidiert: Phloeocharis longipennis FaUvel, $1875=$ P. hummleri Bernhauer, 1915, syn. nov.; Phytosus balticus KraAtz, 1859 = P. holtzi Bernhauer, 1935, syn. nov.; Atheta nigra $($ KraAtz, 1856) $=$ A. biroi Scheerpeltz, 1964, syn. nov.; Domene stilicina $($ Erichson, 1840) $=$ D. lohseiana Bordoni, 1977, syn. nov.; Oedichirus rubronotatus PiC, 1903, revalidiert = O. reitteri Bernhauer, 1908, syn. nov.; Pseudolathra quadricollis (FAuvel, 1875) = P. cretensis Bordoni, 1986, syn. nov. Für Stichoglossa graeca Bernhauer, 1905 wird ein Lectotypus designiert. Zwei Arten werden erstmals aus Griechenland nachgewiesen.

\section{Schlüsselwörter}

Coleoptera, Staphylinidae, Paläarktis, Mittelmeerregion, Griechenland, Kreta, Diversität, Zoogeographie, Inselbiogeographie, Endemismus, Speziation, Taxonomie, neue Synonyme, Revalidierung, Redeskription, Lectotypendesignation, Checkliste, Erstnachweise

\section{Introduction}

As has been shown in several previous studies, the Staphylinidae fauna of Crete is charactized by a remarkable degree of endemism (Assing 2013a, 2015a, 2018c), much more so than the faunas of any of the other East Mediterranean islands. Explanations are found in its topology, its geographic isolation, and in its geological past.

With an area of approximately $8,340 \mathrm{~km}^{2}$ and a west-east extension of about $260 \mathrm{~km}$, Crete is the fifth-largest island of the Mediterranean Sea. The nearest distance from the peninsulas in the northwest to the southernmost tip of the Pelopónnisos is approximately $100 \mathrm{~km}$, that from the northeast to the nearest parts of mainland Turkey nearly $200 \mathrm{~km}$, to the southernmost tip of the Greek island Rhodos about $150 \mathrm{~km}$, and from the south coast to the 
coast of Libya nearly $300 \mathrm{~km}$. Moreover, Crete features numerous mountain ranges, the four major ones being the Lefka Ori in the west, Psiloritis in the centre, and the Dikti range in the east, all of them with several peaks of significantly more than $2,000 \mathrm{~m}$, and the Orno Thriptis in the extreme east. Not only do these mountains ranges guarantee a constant supply of water and moisture from precipitation during the cold and rainy seasons and from melting snow during the warm seasons, they also provide for a remarkable diversity of vegetation covers and microclimates owing to the presence of a wide range of elevations, orientations, and exposures.

Crete has had a history of tremendous changes in size, fragmentation, submergence, and uplift brought about by two major forces, tectonism and eustatism. As a result of the movement and subduction of the African under the Eurasian plate the region now including the Balkans, Crete, and Asia Minor rose to form a joint land mass. Crete subsequently separated from this land mass approximately eight million years ago. Originally forming an archipelago of several low islands, Crete underwent a series of events of partial submersion and subsequent rising, and eventually rose during a period of approximately three million years until it roughly received its present shape and topology. The geological transformations occurring during its geological history have also resulted in an enormous diversity of rock formations on the island (Jolivet \& Brun 2010, Perissoratis \& Conispoliatis 2003, Triantis \& Mylonas 2009). Hence, the geological history together with the topology and the isolation provided for an ideal setting for speciation.

Owing to its long-term isolation and topological heterogeneity, Crete hosts numerous endemic plants and animals. The endemic flora and fauna is composed of three types of endemics: so-called palaeo-endemics (i.e., species that differentiated prior to the splitting of the Aegean land mass and survived only in Crete, e.g., the monotypical plant genus Petromarula), endemics that evolved as a result of vicariance after the separation from the mainland, and species formed owing to in-situ radiation on the island (Cellinese et al. 2009, Triantis \& Mylonas 2009). Unsurprinsingly, endemism is particularly high in poor dispersers, e.g. land snails $(\sim 50 \%)$ and isopods $(\sim 30 \%)$, whereas that of good dispersers such as birds or dragonflies is zero or nearly so (TRIANTIS \& Mylonas 2009). In a study of Campanulaceae, Cellinese et al. (2009) found that the Cretan endemics, which account for $50 \%$ of the total diversity on the island, do not form a monophyletic group, but are scattered across the campanuloid clade. They conclude that most lineages form remnants of an older continental fauna, with only one clade representing the result of in-situ radiation and another one having arrived by dispersal. According to Triantis \& Mylonas (2009), the fauna of eastern Crete is less diverse and more disharmonic than that of western and central Crete.

Despite its relatively easy accessibility for European entomomologists and its long tradition as a touristic destination, the Staphylinidae of Crete had been studied only sporadically until very recently. The first (individual) species of Staphylinidae were described or reported from Crete in the second half of the $19^{\text {th }}$ century (KRAATZ 1857, 1858a, b, Reitter 1885), and Heyden (1884) was the first to provide a list of beetles containing also Staphylinidae (eleven species) collected in spring 1883. Shortly afterwards, OERTzEN (1887) compiled a more comprehensive account of the beetles "Griechenlands und Cretas". SAHLBERG (1903) then published a list of beetles, among them 25 species of Staphylinidae, collected in Crete during a one-week excursion in March, 1899. Several years later, BLATTNÝ \& BLATTNÝ (1916) provided a list of Pselaphinae and Scydmaeninae collected in Crete. Additional, but again individual species were reported in the first half of the $20^{\text {th }}$ century by BRUNDIN (1944), Bernhauer (1915, 1935, 1939); Lokay (1913, 1921), Mařan (1935), and Scheerpeltz (1936). The number of articles containing records of species significantly increased during the period from 1950 to 2012, the vast majority of them representing either taxonomic revisions or synopses of individual genera, tribes, or subfamilies, or taxonomic studies covering more extensive geographic units such as the Mediterranean or the West Palaearctic (see references listed in the checklist of this monograph). Only few articles specifically dealt with the fauna of Crete, most of them containing descriptions of individual species. The only contribution treating a larger number of species is that by SCHEERPELTz (1964), who published a list of 71 identified and two unidentified species collected by L. Biró in 1906. Since 2013, however, numerous species have been recorded from Crete, based on the results of several field trips to Crete, and a first account of the endogean fauna was provided (Assing 2013a, 2015a, 2018c). These three articles alone contain descriptions of 18 species, 17 of them endemic.

The increase in knowledge of the endemic Staphylinidae fauna of Crete since the second half of the $19^{\text {th }}$ century somewhat resembles that of exponential growth (Fig. 1). Up until some twenty years ago, only relatively few endemic species had been known from Crete. Nearly 30 years after the beginning of the exploration of the Cretan Staphylinidae fauna, ReITter (1885) was the first to describe endemics, two species of Pselaphinae, from the island. Approximately 30 years later, four additional species, all of them belonging to the Pselaphinae and Scydmaeninae, were added by Blattný \& BLATTNÝ (1916). Nearly thirty years passed before BRUNDIN (1944) described an endemic species of Atheta ТномSON, 1858. Karaman (1955) and LoHSE \& STEel (1961) subsequently added one endemic species of Tychus LEACH, 1817 and three of Lesteva LATREILle, 1797, respectively, raising the number of Cretan endemics to eleven. Six species were then added in the 1970s by Henri Coiffait (four species), Herbert Franz, and Volker Puthz (one species each). There were no further additions in the 1980s. In the 1990s, eight endemics were described by Roberto Pace (two species), Johannes Frisch, Lothar 
Zerche (one species each), and the author (four species). Thus, at the end of 1999, a total of 26 endemic species was known from the island. This figure was raised to 47 in the following decade, with 21 species described by Claude Besuchet, Arnaldo Bordoni, Giorgio Sabella (one species each), Heinrich Meybohm (three species), and the author (14 species, two of them co-authored by Paul Wunderle). Another 18 endemics were added only very present the results of these field trips, which together yielded 5330 specimens. In addition, unpublished material from several earlier expeditions and other sources is included. Based on these sources and a critical evaluation of literature data, a list of the Staphylinidae of Crete is compiled, allowing for a first comprehensive assessment of the fauna of Crete with a particular focus on the endemic fauna.

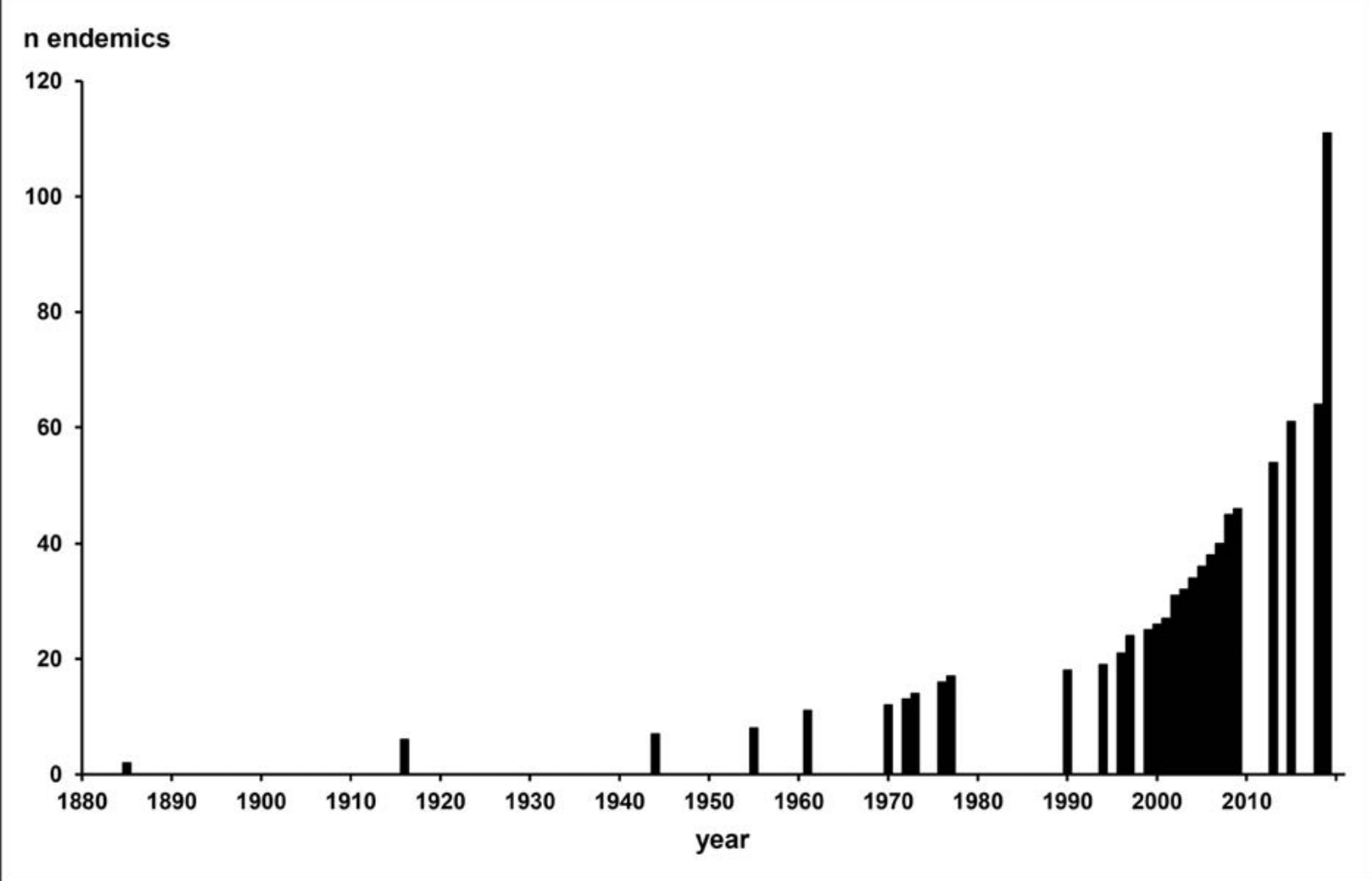

Fig. 1: Cumulative number of named endemic species recorded from Crete from 1880 to 2019.

recently (Assing 2013a, 2015a, 2018c-d), so that, prior to the present monograph, 64 endemic species of Staphylinidae had been recorded from Crete, more than twice as many as are known from the larger East Mediterranean island Cyprus.

It should be noted that several species described from the island and originally considered endemic were subsequently shown to be more widespread or synonyms of widespread species. Such species are not included in the count. Also, to allow for better readability, subspecies are treated as species for the purpose of this section.

Since the previous contributions (Assing 2013a, 2015a), which are mainly based on three field trips conducted by the author in 2012, 2013, and 2014, one of them together with Thomas Forcke and one with Paul Wunderle (Mönchengladbach), five additional field trips to Crete have been conducted, three by the author in winter 2017/2018, spring 2018, and winter 2018/19, and two by Volker Brachat (Geretsried) and Heinrich Meybohm (Großhansdorf) in spring 2018 and spring 2019. One of the objectives of the present study is to
The descriptions of 48 new species, 47 of them endemic, are provided in the second part of the monograph (Assing et al. 2019).

\section{Material and methods}

The material treated in this study is deposited in the following public and private collections:

BMNH The Natural History Museum, London

FMNH Field Museum of Natural History, Chicago

MHNG Muséum d'Histoire Naturelle, Genève (I. Löbl, G. Cuccodoro)

MNB Museum für Naturkunde Berlin (J. Frisch, M. Schülke)

NHMB Naturhistorisches Museum Basel

(C. Germann)

NHMW Naturhistorisches Museum Wien (H. Schillhammer)

SDEI Senckenberg Deutsches Entomologisches Institut, Müncheberg (L. Behne) 
SMF Senckenberg-Museum, Frankfurt

SMNS Staatliches Museum für Naturkunde, Stuttgart (coll. Puthz)

SNM Slovak National Museum, Bratislava

ZMUC Zoological Museum, University of Copenhagen

cAss author's private collection

cBor private collection Arnaldo Bordoni, Firenze

cBra private collection Volker Brachat, Geretsried

cFel private collection Benedikt Feldmann, Münster

cIrm private collection Ulrich Irmler, Kiel

cMat private collection Jan Matějíček, Hradec Králové

cMey private collection Heinrich Meybohm, Großhansdorf

cWun private collection Paul Wunderle, Mönchengladbach

The Pselaphinae and Scydmaeninae (except Cephennium spp.) are deposited in cBra and cMey, respectively. Reference material of the remaining material is deposited in cAss, cFel, cWun, and MNB, if not indicated otherwise.

The morphological studies were conducted using a Stemi SV 11 microscope (Zeiss), a Discovery V12 microscope (Zeiss), and a Jenalab compound microscope (Carl Zeiss Jena). The images were created using digital cameras (Nikon Coolpix 995, Axiocam ERc 5s), Labscope, and Picolay stacking software.

Body length was measured from the anterior margin of the mandibles (in resting position) to the abdominal apex, the length of the forebody from the anterior margin of the mandibles (in resting position) to the posterior margin of the elytra, head length along the middle from the anterior margin of the clypeus (without ante-clypeus), elytral length at the suture from the apex of the scutellum to the posterior margin of the elytra, and the length of the median lobe of the aedeagus from the apex of the ventral process to the base of the aedeagal capsule. The "parameral" side (i.e., the side where the sperm duct enters) is referred to as the ventral, the opposite side as the dorsal aspect.

The material accumulated during the field trips from 2017 to 2019 was collected by sifting litter, grass roots, and moss in various habitats, by turning stones, washing gravel of stream banks, and washing soil. The material examined or communicated from other sources was primarily sifted or hand-collected. The specimens made available by Pier Mauro Giachino and Dante Vailati were caught with subterranean pitfall traps.

Collecting conducted by Volker Brachat, Heinrich Meybohm, and the author mainly focused on habitats where chances of finding endemic species were greatest, i.e., forests and bushland of various kinds and at various altitudes, partly near permanent or temporary streams, as well as high-altitude shrub habitats and grasslands. In addition, the fauna of stream banks and occasionally also that of dung, grass heaps, and beach debris was studied.

\section{Results}

\subsection{Diversity and faunal composition}

The field trips conducted since 2017, a study of previously unpublished material from other previous field trips, and records communicated by various colleagues yielded a total of 281 named (Tab. 1) and at least 21 unnamed species (Tab. 2), the latter represented exclusively by females or belonging to taxa that are currently in taxonomic confusion. Including the new species described in the second part of the monograph (Assing et al. 2019), 115 named species are reported from Crete for the first time. Based on newly examined material and reliable previous records, the Staphylinidae fauna is currently composed of 397 named and at least 21 unnamed species. Previous literature records of more than 50 species are erroneous or are considered doubtful for various reasons (Tab. 4); these species are omitted from the list of Cretan Staphylinidae (Tab. 1). As many as 48 species, 47 of them endemic, are newly described in the second part of the monograph, and six names are synonymized (see section $3.10)$.

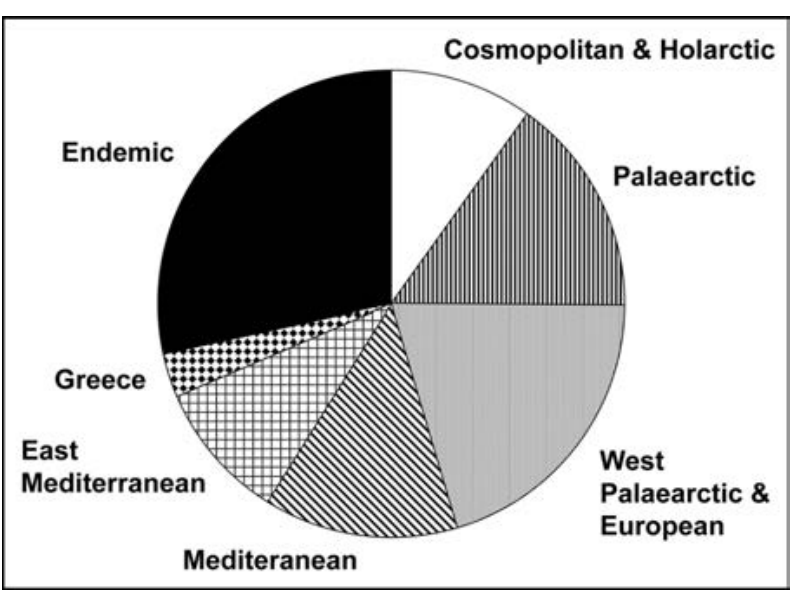

Fig. 2: Zoogeographic composition of the Staphylinidae fauna of Crete (only named species).

Considering that numerous species have been recorded only once, that various microhabitats which are known to host numerous specialized Staphylinidae species (e.g., nests of mammals and birds, mushrooms, bark of dead trees, compost, etc.) have not, or not thoroughly, been studied, and in view of the methodology used it can be assumed that the known fauna is still somewhat incomplete. A substantial number of species may still be added particularly through methods suitable for collecting flying insects (flight interception traps, car-nets) and for Staphylinidae with a subterranean reproduction habitat and short seasonal epigeic dispersal periods (pitfall traps).

Nearly half of the named species recorded from Crete are widespread, with Cosmopolitan and Holarctic elements accounting for approximately $10 \%$, species with transPalaearctic distributions for $15 \%$, and West Palaearctic and European elements for $20 \%$ of the fauna. Another 
$23 \%$ are widespread Mediterranean species, with $10 \%$ confined to the East Mediterranean region. Twelve species ( $3 \%$ ) have been recorded only from Greece, five of them exclusively from Aegean islands. Remarkably, nearly one-third of the fauna is endemic (Fig. 2). For more details on the composition of the endemic fauna see section 3.6.

Few, evidently adventive species appear to have arrived on the island only recently, e.g., Leptomastax bipunctata and Stenus turbulentus. The former is distributed in Turkey and the Balkans, but known from only one locality in West Crete. The latter is widespread and common in the East Mediterranean, but had never been recorded from Crete until 2018, when it was collected in several localities. It seems most unlikely that a common inhabitant of the litter layer like S. turbulentus should have been overlooked on previous field trips.

\subsection{Checklist of the Staphylinidae of Crete}

The below checklist summarizes the Staphylinidae recorded from Crete, based on unpublished results of various previous field trips and on reliable previously published records. In the localities column, the number of specimens is given in parentheses behind the locality number. Previous literature records are mostly scattered in articles without specific focus on the fauna of Crete and consequently bound to be somewhat incomplete, despite considerable efforts at searching the relevant literature. Literature records that are at least likely to be based on misidentification, old records of species that are reliably identified only based on an examination of the primary sexual characters, records of "species" that were subsequently discovered to represent two or more species, or records that are doubful for other reasons are omitted and listed separately in Tab. 3. For Pselaphinae, Steninae, and Scydmaeninae, such omissions are based on BRACHAT (pers. comm.), Puthz (pers. comm.), and Меувонм (pers. comm.), respectively. Unnamed (unidentified and undescribed) species are listed in Tab. 2.

Tab. 1: Checklist of the named Staphylinidae recorded from Crete. Details on the localities/samples and the references are provided at the end of the checklist. - Explanation of symbols: ${ }^{\star}$ endemic to Crete (island endemic); ${ }^{\star \star}$ locally endemic (i.e., restricted to individual mountains or regions in Crete); \# taxonomic and zoogeographic status unclear.

\begin{tabular}{|c|c|c|}
\hline Species & Localities/samples & References \\
\hline \multicolumn{3}{|l|}{ Omali ina e } \\
\hline Acidota cruentata MANnERHEIM, 1830 & $110(1)$ & A13a \\
\hline Anthobium atrocephalum (GYLLENHAL, 1827) & $137(2)$ & A13a \\
\hline${ }^{*}$ Boreaphilus fuelscheri ZERCHE, 1990 & $\begin{array}{l}\text { 4(1), 109a(2), 119(1), 123(3), 128(1), } \\
134(1), 136(1), 180(1)\end{array}$ & A02, A13a, A15a, Z90 \\
\hline${ }^{* *}$ Boreaphilus meybohmi Assing, 2002 & 135(1), 137(1), 138(2), 172(1), 182(1) & A02, A15a \\
\hline Boreaphilus velox (HEER, 1839) & & A15a, Z90 \\
\hline Deliphrosoma fratellum (RoTTENBERG, 1874) & & A15a, Z91 \\
\hline Dropephylla cretica Jaszay \& HLAVÁč, 2006 & $106(3)$ & JH06 \\
\hline Hypopycna rufula (ERICHson, 1840) & 194(1) & \\
\hline Lesteva brondeeli LOHSE \& STEEL, 1961 & & LS61, P79 \\
\hline $\begin{array}{l}{ }^{*} \text { Lesteva longoelytrata cretica LOHSE \& STEEL, } \\
1961\end{array}$ & $88(4), 90(2)$ & $\begin{array}{l}\text { A15a, C76, Kz58b }{ }^{57)} \text {, LS61, } \\
\text { O87, P79 }\end{array}$ \\
\hline${ }^{*}$ Lesteva nitidicollis LOHSE \& STEEL, 1961 & & LS61, P79 \\
\hline${ }^{*}$ Lesteva szekessyi LOHSE \& STEEL, 1961 & & LS61 \\
\hline Omalium cinnamomeum KraATZ, 1857 & $\begin{array}{l}180(1), 182(1), 47(1), 58(48), 64(1), \\
73(1), 78(1), 95 a(1), 108(2), 119(2), \\
128(1), 136 a(1), 144(1), 147(1), 156(1), \\
176(1) ; 195(2), 202(8), 203(9), 204(1), \\
205(18), 206(4)\end{array}$ & A13a, A15a, O87 \\
\hline Omalium excavatum STEPHENS, 1834 & $\begin{array}{l}\text { 41a(1), 121(1), 134(1), 136(1), 139(5), } \\
\text { 205(1), 211(1), 216(5) }\end{array}$ & A13a, A15a, C76 \\
\hline $\begin{array}{l}\text { Omalium riparium impar Mulsant \& REY, } \\
1861\end{array}$ & & $\mathrm{Sa03}$ \\
\hline Omalium rhodicum Assing \& ZANETTI, 2013 & & A15a \\
\hline
\end{tabular}




\begin{tabular}{|c|c|c|}
\hline Species & Localities/samples & References \\
\hline Omalium rivulare (PAYKULL, 1789) & 144(1), 211(1) & \\
\hline Omalium rugatum Mulsant \& REY, 1880 & 4(1), 180(1), 202(1) & A13a, A15a \\
\hline \multicolumn{3}{|l|}{ Proteinina e } \\
\hline Megarthrus bellevoyei SAULCY, 1862 & & A13a, Ho63 \\
\hline Megarthrus denticollis (BECK, 1817) & 219(1) & \\
\hline Megarthrus depressus (PAYKuLL, 1789) & $34(1), 43(1), 76(2), 134(3)$ & \\
\hline Megarthrus prosseni Schatzmayr, 1904 & $180(1)$ & \\
\hline Metopsia similis ZERCHE, 1998 & 141(6), 144(1), 169(2), 180(2) & \\
\hline Proteinus atomarius ERICHSON, 1840 & $\begin{array}{l}\text { 41a(2), 76(1), 152(1), 150(3), 154(1), } \\
167(1), 168(1), 169(1), 193(2)\end{array}$ & A15a \\
\hline${ }^{*}$ Proteinus creticus Assing, 2004 & $\begin{array}{l}28(3), 41 \mathrm{a}(7), \quad 62(1), 122(3), 123(6) \\
124(1), 131(6), 134(2), 138(2), 147(1), \\
149(4), 153(1), 159(1), 161(3), 165(1), \\
170(1), 175(2), 180(1), 193(1), 211(2), \\
214(1)\end{array}$ & A04b, A13a, A15a \\
\hline Proteinus ovalis STEPHENS, 1834 & $160(1), 161(4)$ & A13a, A15a \\
\hline \multicolumn{3}{|l|}{ Micropeplinae } \\
\hline Micropeplus fulvus ERICHSON, 1840 & $74(1), 192(1)$ & \\
\hline Micropeplus porcatus (PAYKULL, 1789) & & Kz58b, O87 \\
\hline Micropeplus staphylinoides (MARSHAM, 1802) & 145(1), 166(2), 168(1), 180(3) & O87 \\
\hline \multicolumn{3}{|l|}{ Pselaphinae } \\
\hline${ }^{* *}$ Afropselaphus assingi BRACHAT, 2019 & $171(2)$ & ABM19 \\
\hline${ }^{* *}$ Afropselaphus diktianus BRACHAT, 2019 & & ABM19 \\
\hline${ }^{* *}$ Afropselaphus dornfeldae BRACHAT, 2019 & & ABM19 \\
\hline${ }^{* *}$ Afropselaphus thripticus BRACHAT, 2019 & $5(1)$ & ABM19 \\
\hline${ }^{* *}$ Amauronyx askifouicus BRACHAT, 2019 & $70(5)$ & ABM19 \\
\hline${ }^{* *}$ Amauronyx chanianus BRACHAT, 2019 & $53(1), 53 a(1)$ & ABM19 \\
\hline${ }^{* *}$ Amauronyx occidens BRACHAT, 2019 & $96(3), 97(2)$ & ABM19 \\
\hline $\begin{array}{l}{ }^{* *} \text { Amauronyx paganettii BLATTNÝ \& BLATTNÝ, } \\
1916\end{array}$ & 101(1) & BB16 \\
\hline $\begin{array}{l}\text { *Batrisodes paganettii BLATTNÝ \& BLATTNÝ, } \\
1916\end{array}$ & $\begin{array}{l}\text { 130(1), 144(2), 149(4), 154(1), 157(1), } \\
\text { 229(2), 231(2), 247(3) }\end{array}$ & BB16 \\
\hline Brachygluta cavernosa (SAULCY, 1876) & $237(2)$ & A13a ${ }^{7)}, A 15 a$, SBBB04 \\
\hline Brachygluta foveola (MоTSCHULSKY, 1840) & & $\mathrm{BB}^{6} 6^{64)}, \mathrm{SBBB} 04$ \\
\hline *Brachygluta gnosiaca BEsUCHET, 2004 & & SBBB04 \\
\hline Brachygluta helferi longispina (REITTER, 1884) & $234(1)$ & Kz86a, O87, SBBB04 \\
\hline Brachygluta ochanensis (REITTER, 1909) & $\begin{array}{l}\text { 123(1), 128(4), 136(1), 137(4), 164(29), } \\
\text { 171(8), 172(11), 174(4),177(4), 180(3), } \\
233(1), 242(1), 245(2), 246(1), 251(1)\end{array}$ & A13a, A15a \\
\hline Brachygluta paludosa (PEYRon, 1858) & 232(1), 240(4) & SBBB04 \\
\hline Brachygluta tibialis (AuBÉ, 1844) & $240(4), 243(1)$ & BB16, O87 \\
\hline Brachygluta xanthoptera (REICHENBACH, 1816) & $\begin{array}{l}88(1), \quad 89(2), \quad 90(7), \quad 229(1), \quad 239(13), \\
249(7)\end{array}$ & \\
\hline Bryaxis bulbifer (REICHENBACH, 1816) & & BB16 \\
\hline
\end{tabular}




\begin{tabular}{|c|c|c|}
\hline Species & Localities/samples & References \\
\hline **Bryaxis meybohmianus BRACHAT, 2019 & $\begin{array}{l}\text { 10(3), 36(3), 43(19), 52(2), 53(1), 54(2), } \\
\text { 60(2), 67(1), 75(1), 78(1),79(1), 80(1), } \\
\text { 100(3), 135(6), 136(2), 137(2), 142(2), } \\
\text { 144(6), 149(4), 151(1), 153(1), 168(3), } \\
\text { 173(6), 175(1) }\end{array}$ & ABM19 \\
\hline${ }^{*}$ Bythinus creticus BRACHAT, 2019 & & ABM19 \\
\hline${ }^{*}$ Claviger oertzeni ReITteR, 1885 & $248(9)$ & O87, R85 \\
\hline Ctenistes palpalis REICHENBACH, 1816 & 120(1), 235(1), 245(2) & \\
\hline Enoptostomus globulicornis (Мотsсн., 1851) & 71(2), 235(4) & A15a \\
\hline${ }^{\star}$ Euplectus assingi BRACHAT, 2019 & $13(2), 15(1), 17(3), 18(3), 85(1), 157(3)$ & ABM19 \\
\hline Euplectus karstenii (REICHENBACH, 1816) & & BB16 \\
\hline Euplectus kirbii kirbii DENNY, 1825 & $238(1), 250(3)$ & $\mathrm{BB} 16^{63)}$ \\
\hline Euplectus signatus (REICHENBACH, 1816) & & BB16 \\
\hline Euplectus verticalis ReITteR, 1884 & $254(1)$ & \\
\hline 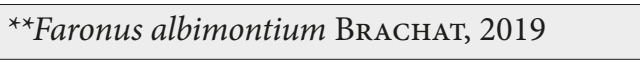 & $138(1), 174(3)$ & ABM19 \\
\hline${ }^{* *}$ Faronus lefkamontium BRACHAT, 2019 & & ABM19 \\
\hline${ }^{* *}$ Faronus meybohmi BRACHAT, 2019 & $32(1), 170(5), 171(3), 174(6), 175(2)$ & ABM19 \\
\hline Faronus parallelus BESUCHET, 1958 & $180(7)$ & BB16 ${ }^{77)}$ \\
\hline Meliceria acanthifera (REITTER, 1884) & $96 \mathrm{a}(2), 146(1), 236(1)$ & \\
\hline Pselaphus acuminatus Мотschulsky, 1835 & $243(1), 245(1)$ & $\mathrm{Kz} 58 \mathrm{a}^{62)}, \mathrm{O} 87^{62)}$ \\
\hline $\begin{array}{l}\text { Pseudoplectus perplexus (JACQUELIN DU VAL, } \\
\text { 1854) }\end{array}$ & $240(3)$ & $\mathrm{BB} 16^{61)}, \mathrm{Be} 55$ \\
\hline Reichenbachia chevrieri (AuBÉ, 1844) & $240(1), 243(3), 252(3)$ & Kz58a, O87 \\
\hline Rybaxis longicornis (LEACH, 1817) & $240(1)$ & $\mathrm{Kz} 58 \mathrm{a}^{60)}, \mathrm{O} 87^{60)}$ \\
\hline Tribatus creticus ReItTeR, 1884 & $38(1), 71(1), 120(1), 160(6)$ & A13a, A15a, O87, \\
\hline Trissemus montanus (SAULCY, 1876) & $255(5)$ & \\
\hline${ }^{* *}$ Tychus chanianus BRACHAT, 2019 & $144(2)$ & ABM19 \\
\hline${ }^{*}$ Tychus creticus ReITteR, 1885 & & BB16, R85, Sa03, Sb02 \\
\hline Tychus dalmatinus ReITter, 1880 & $26(1), 243(1)$ & SBB98 \\
\hline${ }^{* *}$ Tychus lagrecai SABELLA, 2002 & $159(1)$ & A13a ${ }^{8)}, A 15 a$, Sb02 \\
\hline${ }^{*}$ Tychus reitterianus LÖBL, 1998 & $\begin{array}{l}\text { 9(2), 12(1), 28(1), 40(1), 41a(2), 53a(5), } \\
\text { 65(3), 69(2), 72(1), 73(1), 79(1), 85(6), } \\
94(5), 95(2), 102(1), 123(3), 128(5), \\
130(3), 143(1), 144(4), 149(6), 151(2), \\
155(3), 157(4), 159(3), 160(1), 161(2), \\
162(3), 166(2), 167(2), 168(1), 171(2), \\
183(2), 191(1), 228(2), 230(2), 231(2), \\
241(1), 244(3), 246(2), 250(1), 253(1)\end{array}$ & $\begin{array}{l}\text { A13a, Ka55, Lö98, Sb02, } \\
\text { SBB98 }\end{array}$ \\
\hline Tyrus peyroni SAULCY, 1874 & $248(1)$ & O87 ${ }^{59)}$ \\
\hline \multicolumn{3}{|l|}{ Phloeocharinae } \\
\hline Phloeocharis longipennis FAUVEL, 1875 & $127(2)$ & $\mathrm{Br} 15^{69)}, \mathrm{O} 87^{68)}$ \\
\hline \multicolumn{3}{|l|}{ Tachyporinae } \\
\hline Cilea silphoides (LinnAEus, 1767) & & Sz64 \\
\hline Lordithon exoletus (ERICHSON, 1839) & $\begin{array}{l}\text { 41a(1), 96a(1), 159(1), 172(1), 193(1), } \\
\text { 212(1) }\end{array}$ & Ho67, O87 \\
\hline Lordithon thoracicus (FABRICIUS, 1777) & $142(1), 154(1)$ & \\
\hline
\end{tabular}




\begin{tabular}{|c|c|c|}
\hline Species & Localities/samples & References \\
\hline Mycetoporus baudueri Mulsant \& Rey, 1875 & & A13a \\
\hline Mycetoporus glaber glaber (SPERK, 1835) & $\begin{array}{l}\text { 64(1), } 85(2), \quad 114(1), \quad 119(2), \quad 142(1), \\
149(1), 164(1), 165(1)\end{array}$ & \\
\hline Mycetoporus ignidorsum EPPELSHEIM, 1880 & $5(1), 32(1), 123(2), 220(1)$ & A13a, A15a \\
\hline Mycetoporus laticeps FAGEL, 1965 & & Sc19 \\
\hline Mycetoporus nigricollis (STEPHENS, 1835) & 203(8), 205(9), 207(6) & SK00 \\
\hline Mycetoporus reichei (PANDELLÉ, 1869) & 11(2), 32(1), 179(1), 209(1) & A15a \\
\hline $\begin{array}{l}\text { Mycetoporus silvaticus IABLOKOFF-KHNZO- } \\
\text { RIAN, } 1962\end{array}$ & & $A 13 a^{82)}, A 15 a^{82)}$ \\
\hline Mycetoporus simillimus FAGEL, 1965 & $\begin{array}{l}28(3), 30(1), 34(1), 35(1), 44(1), 45(1), \\
53 \mathrm{a}(2), 73(1), 74(1), 80(3), 80 \mathrm{a}(1), \\
94 \mathrm{a}(6), 121(1), 123(1), 124(3), 129(2), \\
130(1), 133(2), 135(1), 136(1), 138(1), \\
140(1), 141(1), 142(1), 143(2), 144(1), \\
147(2), 150(2), 153(1), 158(1), 159(2), \\
165(9), 170(4), 172(1), 175(2), 183(2), \\
193(1)\end{array}$ & $A 13 a^{1)}, A 15 a^{1)}, S c 19$ \\
\hline $\begin{array}{l}\text { Parabolitobius inclinans (GRAVENHORST, } \\
\text { 1806) }\end{array}$ & & O87 \\
\hline *Sepedophilus creticus ScHüLKE, 2019 & 112(1), 161(1), 220(2), 223(2) & ABM19, $\mathrm{P} 80^{80)}$ \\
\hline Sepedophilus immaculatus (STEPHENs, 1832) & $\begin{array}{l}41 \mathrm{a}(1), 68(1), 149(5), 150(2), 153(1), \\
164(1), 180(2)\end{array}$ & C76, Sz64 \\
\hline Tachinus bonvouloiri PANDELLÉ, 1869 & & A15a \\
\hline Tachyporus abner SAULCY, 1865 & & A13a, A15a \\
\hline Tachyporus caucasicus (Kolenati, 1846) & 20(1), 23(2), 91(1), 92a(1), 99(1), 100(2), & $\mathrm{A} 13 \mathrm{a}, \mathrm{C} 80^{54)}, \mathrm{O} 87^{36)}$ \\
\hline Tachyporus hypnorum (FABRICIUs, 1757) & $\begin{array}{l}\text { 95(1), 195(2), 201(2), 205(1), 206(6), } \\
\text { 223(1) }\end{array}$ & A13a, Kz58a, O87, Sz64 \\
\hline Tachyporus nitidulus (FABRICIUs, 1781) & $\begin{array}{l}95(1), 95 a(2), 96 a(1), 104(1), 108(1) \\
109 b(1), 110(7), 111(2), 112(7), 113(1) \\
118(1), 119(4), 120(2), 125(1), 161(2), \\
165(1), 170(1), 171(1), 187(2), 195(2) \\
201(2), 205(1), 206(1)\end{array}$ & $\begin{array}{l}\text { A13a, A15a, C76, Kz58a }{ }^{55)} \\
\text { O87, Sz64 }\end{array}$ \\
\hline \multicolumn{3}{|l|}{ Habrocerinae } \\
\hline Habrocerus pisidicus KoRGE, 1971 & 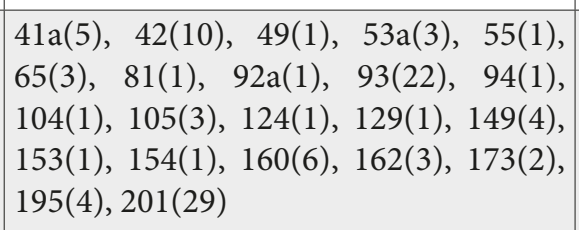 & A08b, A13a, AW95, O87 ${ }^{35)}$ \\
\hline \multicolumn{3}{|l|}{ Aleocharinae } \\
\hline Acrotona nigerrima (AuBÉ, 1850) & $75 a(1)$ & \\
\hline Acrotona parvula (MANNERHEIM, 1830) & $75 a(46), 76(16)$ & \\
\hline Aleochara albopila (Mulsant \& REy, 1852) & $199(1)$ & \\
\hline Aleochara bipustulata (LinnaEus, 1760) & $75 a(1)$ & A13a, C76, Sz64 \\
\hline Aleochara crassa BAudi di SELve, 1848 & & Sz64 \\
\hline Aleochara curtula (GoEze, 1777) & & Sz64 \\
\hline Aleochara grisea KRAATZ, 1856 & & A95 \\
\hline Aleochara haematoptera KRAATZ, 1858 & $196(1), 219(2)$ & \\
\hline Aleochara intricata MANNERHEIM, 1830 & & Sz64 \\
\hline
\end{tabular}




\begin{tabular}{|c|c|c|}
\hline Species & Localities/samples & References \\
\hline Aleochara laticornis KRAATZ, 1856 & $28(1)$ & C76 \\
\hline Aleochara milleri KRAATZ, 1862 & & Sz64 \\
\hline Aleochara moesta GravenHORST, 1802 & $76(1)$ & Sz64 \\
\hline Aleochara puberula KLUG, 1832 & & Sz64 \\
\hline Aleochara tristis GRAVENHORST, 1806 & & Kz58a, O87, Sz64 \\
\hline $\begin{array}{l}{ }^{*} \text { Alevonota cretica Assing \& WUNDERLE, } \\
2008\end{array}$ & $\begin{array}{l}3(2), 81(1), 95(5), 96(1), 125(1), 159(1) \\
160(3), 163(1), 169(1), 174(1), 181(1)\end{array}$ & A15a, AW08 \\
\hline *Aloconota brachyptera Assing, 2013 & & A13a, A15a \\
\hline Aloconota gregaria (ERICHSON, 1839) & $120(1)$ & He84, O87 \\
\hline${ }^{*}$ Aloconota minoica PACE, 2002 & $89(1), 90(2)$ & A13a, Pa02 \\
\hline Aloconota planifrons (WATERHOUSE, 1863) & $88(1)$ & \\
\hline Aloconota subgrandis (BRUNDIN, 1954) & $18 \mathrm{a}(3), 36 \mathrm{a}(1), 68(1), 154(1)$ & \\
\hline${ }^{\star}$ Amischa cretica Assing, 2019 & $3(1), 175(10)$ & A13a ${ }^{74)}, \mathrm{ABM} 19$ \\
\hline Amischa forcipata (Mulsant \& REY, 1873) & $94 \mathrm{a}(2)$ & \\
\hline Atheta aeneicollis (SHARP, 1869) & $\begin{array}{l}8(1), 18 \mathrm{a}(14), 23(1), 25(2), 34(1), 35(1), \\
37(1), 41(4), 41 \mathrm{a}(24), 42(4), 47(1), \\
50(2), 51(1), 53(1), 53 \mathrm{a}(5), 57(1), 61(1), \\
62(1), 67(2), 68(1), 70(1), 72(1), 74(4), \\
74 \mathrm{a}(1), 76(5), 80(1), 80 \mathrm{a}(2), 82(1), \\
84(4), 93(18), 95 \mathrm{a}(2), 97(1), 99(2), \\
103(1), 105(1), 109 \mathrm{~b}(2), 118(1), 121(8), \\
122(2), 123(3), 124(2), 128(1), 129(2), \\
131(3), 133(1), 140(1), 142(2), 144(5), \\
145(1), 147(7), 149(5), 150(1), 151(1), \\
155(1), 158(5), 159(4), 163(1), 164(2), \\
166(3), 167(2), 169(2), 170(5), 171(2), \\
176(1), 177(4), 181(1), 195(10), 201(4), \\
203(2), 204(10), 206(19), 218(1)\end{array}$ & A13a, A15a, Sa03 ${ }^{47)}$ \\
\hline Atheta amicula (STEPHENs, 1832) & $\begin{array}{l}41 \mathrm{a}(1), 75 \mathrm{a}(1), 76(19), 90(1), \quad 121(1), \\
166(1)\end{array}$ & A13a, A15a, O87 \\
\hline Atheta atramentaria (GyLLENHAL, 1810) & $75 a(31)$ & \\
\hline Atheta atricolor (SHARP, 1869) & $75 a(1), 76(1)$ & \\
\hline Atheta coriaria (KRAATZ, 1856) & & O87, Sa03 \\
\hline${ }^{*}$ Atheta cretica BRUNDIN, 1944 & $90(4)$ & $\begin{array}{l}\text { A13a, A15a, Bn44, Kz58a }{ }^{34)} \\
\text { O87 }\end{array}$ \\
\hline${ }^{*}$ Atheta digitalis Assing, 2019 & $135(2)$ & ABM19 \\
\hline Atheta fimorum (BRISOUT, 1860) & $75 a(3)$ & \\
\hline Atheta fossiceps ScheERPLETz, 1964 & $27(2)$ & Sz64 \\
\hline Atheta inquinula (GRAVENHORst, 1802) & & Sa03, Sz64 \\
\hline Atheta longicornis (GRAVENHORST, 1802) & $75 a(6), 76(57)$ & Sz64 \\
\hline Atheta luctuosa (Mulsant \& ReY, 1853) & & A15a \\
\hline Atheta mucronata (KRAATZ, 1859) & $169(1)$ & \\
\hline Atheta nigra (KRAATZ, 1856) & $76(14)$ & A06, A13a, A15a, Sz64 ${ }^{70)}$ \\
\hline Atheta oblita (ERICHSON, 1839) & $177(1)$ & A13a \\
\hline Atheta occulta (ERICHSON, 1837) & & $A 13 a^{9)}, A 15 a, O 87$ \\
\hline Atheta ravilla (ERICHSON, 1839) & $76(3)$ & \\
\hline Atheta sodalis (ERICHSON, 1837) & & O87 \\
\hline
\end{tabular}




\begin{tabular}{|c|c|c|}
\hline Species & Localities/samples & References \\
\hline Atheta triangulum (KRAATz, 1856) & & $\mathrm{O} 87, \mathrm{Sa} 03$ \\
\hline Atheta trinotata (KRAATZ, 1856) & $128(1), 165(2), 216(1)$ & \\
\hline 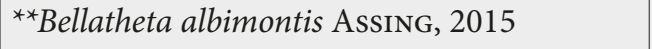 & $32(12), 182(2)$ & A15a \\
\hline${ }^{* *}$ Bellatheta idana Assing, 2015 & & A15a \\
\hline Bolitochara obliqua ERICHSON, 1837 & $96 \mathrm{a}(23), 102(1), 108 \mathrm{a}(4), 150(2), 167(1)$ & A14b, O87 \\
\hline Caloderina hierosolymitana (SAULCY, 1865) & $112(1)$ & A15a, Sa03 \\
\hline Cordalia obscura (GRAVENHORst, 1802) & $75 \mathrm{a}(1), 76(11), 166(1), 208(1)$ & Sz64 \\
\hline${ }^{* *}$ Cousya candica Assing, 2019 & $108(5), 126(1), 128(1)$ & ABM19 \\
\hline Cousya defecta (Mulsant \& ReY, 1875) & 176(1), 180(1), 210(1) & A18a \\
\hline $\begin{array}{l}\text { Cousya nigrata (FAIRMAIRE \& LABOULBÈnE, } \\
1856 \text { ) }\end{array}$ & 181(1), 205(1) & A18a \\
\hline Cypha graeca Assing, 2004 & & A04b, A13a \\
\hline Dinusa cretica Assing, 2013 & $27(1)$ & A13a, A15a \\
\hline${ }^{*}$ Drusilla cretica Assing, 2005 & 71(1), 139(1), 164(1), 174(1) & A05b, A13a, A15a \\
\hline Falagria caesa ERICHSON, 1837 & $76(2)$ & $\mathrm{Kz} 58 \mathrm{a}^{23)}, \mathrm{O} 87^{23)}, \mathrm{Sz} 64^{23)}$ \\
\hline${ }^{* *}$ Geostiba albimontis Assing, 2007 & $52(1), 53(1), 72(3), 165(8), 170(23)$ & A07d \\
\hline${ }^{* *}$ Geostiba diktiana Assing, 2013 & & A13a \\
\hline${ }^{* *}$ Geostiba exsecta Assing, 1999 & $161(9)$ & A99, A13a \\
\hline${ }^{* *}$ Geostiba icaria PACE, 1996 & 144(1), 184(1) & A99, A01, A15a, P96 \\
\hline${ }^{* *}$ Geostiba idaea PACE, 1996 & 23(1), 133(1), 134(22), & A99, A15a, P96 \\
\hline${ }^{* *}$ Geostiba inexsecta Assing, 2019 & $103(1), 104(11)$ & ABM19 \\
\hline${ }^{* *}$ Geostiba meybohmi Assing, 2000 & $\begin{array}{l}7(1), \quad 15(2), \quad 28(2), \quad 84(5), \quad 108(81) \\
109 \mathrm{a}(28), 114(108), 119(125), 126(5) \\
127(4), 128(5), 159(1), 180(4), 181(3)\end{array}$ & A00, A01, A13a \\
\hline Geostiba oertzeni (EPPELSHEIM, 1888) & $\begin{array}{l}1(23), 2(22), 6(1), 9(1), 12(12), 13(3), \\
16(1), 17(74), 18(8), 19(9), 20(4), 22(1), \\
25(2), 30(8), 31(6), 32(160), 34(1), \\
36(7), 40(7), 41 a(2), 44(11), 45(3), \\
50(2), 51(1), 62(65), 64(14), 67(26), \\
78(4), 79(5), 80(13), 80 a(2), 81(1), \\
82(3), 85(1), 92 b(2), 95(4), 97(4), 98(1), \\
103(2), 107(3), 109(2), 115(1), 129(4), \\
130(14), 131(3), 135(39), 136 a(6), \\
137(4), 138(7), 144(1), 153(3), 157(28), \\
171(8), 172(10), 174(1), 182(60), 188(1), \\
190(5), 192(1)\end{array}$ & $\begin{array}{l}\text { A99, A01, A13a, A15a, } \\
\text { P96 }^{26)}\end{array}$ \\
\hline${ }^{* * G e o s t i b a}$ paulexsecta Assing, 2015 & & A15a \\
\hline${ }^{* *}$ Geostiba thryptisensis Assing, 2001 & $\begin{array}{l}\text { 4(1), 5(2), 6(3), 123(4), 131(1), 177(1), } \\
178(3), 179(34)\end{array}$ & A01, A13a \\
\hline Gnypeta carbonaria (MANNERHEIM, 1830) & $223(1)$ & \\
\hline Gnypeta rubrior Toтtenнaм, 1939 & 208(1), 223(1) & $\mathrm{A} 06, \mathrm{C} 76^{29)}$ \\
\hline Gyrophaena affinis (MANNERHEIM, 1830) & 91(1), 92b(1) & \\
\hline Halobrecta flavipes Tномson, 1861 & $199(1)$ & \\
\hline Heterota plumbea (WATERHOUsE, 1858) & 199(28) & \\
\hline Hydrosmecta fluviatilis (KRAATZ, 1854) & $36 a(1), 90(1)$ & A13a ${ }^{67)}$, App \\
\hline Hydrosmecta insularum Assing, 2019 & 88(75), 89(12), 90(85) & ABM19 \\
\hline
\end{tabular}




\begin{tabular}{|c|c|c|}
\hline Species & Localities/samples & References \\
\hline Hydrosmecta longula (HEeR, 1839) & $\begin{array}{l}\text { 88(13), 89(20), 90(100), 194(2), 196(4), } \\
\text { 208(10) }\end{array}$ & A06, A13a ${ }^{67)}, A 15 a^{67)}, A p p$ \\
\hline Ischnoglossa prolixa (GRAVENHORST, 1802) & & O87 \\
\hline Liogluta longiuscula (GRAVEnHORST, 1802) & $\begin{array}{l}30(3), 41 \mathrm{a}(1), 50(1), 62(4), 67(1), 68(1) \\
85(8), 89(32), 90(4), 119(1), 131(7) \\
133(4), 149(2), 154(1), 158(3), 160(4) \\
161(1), 165(1), 175(1), 176(1), 219(1)\end{array}$ & $\begin{array}{l}\text { A13a, A15a, C76, Sa03 }{ }^{46)} \text {, } \\
\text { Sz64 }\end{array}$ \\
\hline Meotica parasita Mulsant \& ReY, 1873 & 18(1), 90(1), 94(2), 95(1), 166(1) & AV19 \\
\hline${ }^{*}$ Myllaena cretica Assing, 2018 & $90(10)$ & A18d \\
\hline Myllaena intermedia ERICHSON, 1837 & $\begin{array}{l}\text { 36a(1), 94a(5), 166(1), 197(2), 219(1), } \\
220(1)\end{array}$ & \\
\hline${ }^{* *}$ Myrmecopora elisa Assing, 1997 & $27(8), 29(3), 86(5), 120(7)$ & A97a, A13a, A15a \\
\hline${ }^{* *}$ Myrmecopora fornicata Assing, 1997 & $56(4)$ & A97a, A15a \\
\hline Myrmecopora fugax (ERICHSON, 1839) & $76(1)$ & A97a \\
\hline${ }^{* *}$ Myrmecopora idana Assing, 2013 & & A13a, A15a \\
\hline Myrmecopora laesa (ERICHSON, 1839) & & A97a, A15a \\
\hline${ }^{* *}$ Myrmecopora plana Assing, 1997 & $71(1)$ & A97a, A15a \\
\hline Myrmecopora sulcata (KIESENWETTER, 1850) & & $\mathrm{Sa} 03$ \\
\hline${ }^{* *}$ Myrmecopora thriptica Assing, 2015 & & $\mathrm{~A} 13 \mathrm{a}^{2)}, \mathrm{A} 15 \mathrm{a}$ \\
\hline $\begin{array}{l}\text { Nehemitropia lividipennis (MANNERHEIM, } \\
\text { 1830) }\end{array}$ & $76(3), 220(1)$ & $\mathrm{O} 87^{24)}, \mathrm{Sz} 64^{24)}$ \\
\hline${ }^{*}$ Ocalea cretica CoIfFAIT, 1976 & 199(16), 205(1) & A13a ${ }^{3)}$, A15a, C76, Sz64 ${ }^{3)}$ \\
\hline Oligota muensteri BERNHAUER, 1923 & 18(1), 84(1), 141(1), 180(6) & \\
\hline Oligota parva KRAATZ, 1862 & & Sz64 \\
\hline Oligota pumilio KIESENWETTER, 1858 & 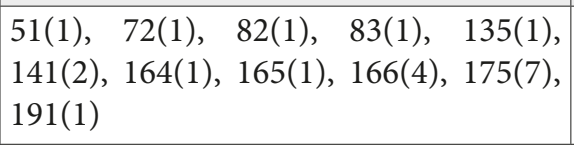 & \\
\hline Outachyusa raptoria (WoLlaston, 1854) & $219(1)$ & \\
\hline Oxypoda bimaculata BAUDI, 1870 & & A13a, A15a \\
\hline *Oxypoda bimontium Assing, 2019 & $90(1)$ & ABM19 \\
\hline Oxypoda brevicornis (STEPHENs, 1832) & $36 a(1), 149(1)$ & \\
\hline Oxypoda carbonaria (HEeR, 1841) & & $\mathrm{Kz} 58 \mathrm{a}^{25)}, \mathrm{Sz} 64^{25)}$ \\
\hline${ }^{* *}$ Oxypoda cretica Assing, 2006 & $181(1)$ & A06, A13a \\
\hline Oxypoda flavicornis KRAATZ, 1856 & $108(4), 119(6)$ & \\
\hline Oxypoda haemorrhoa (MANNERHEIM, 1830) & $120(1)$ & A15a \\
\hline${ }^{* *}$ Oxypoda idana Assing, 2013 & 206(1) & A13a \\
\hline Oxypoda lesbia Assing, 2005 & & $\mathrm{~A} 13 \mathrm{a}^{4)}, \mathrm{A} 15 \mathrm{a}$ \\
\hline Oxypoda lurida Wollaston, 1857 & $\begin{array}{l}\text { 50(1), 75a(1), 141(2), 147(2), 161(1), } \\
\text { 205(1), }\end{array}$ & A13a, A15a \\
\hline Oxypoda rectacia Assing, 2019 & $\begin{array}{l}44(1), 44 a(1), \quad 53 a(2), 80(1), 94(4), \\
104(1), 105(1), 115(1), 136(1), 137(1), \\
139(1), 143(2), 144(4), 145(2), 147(1) \\
149(3), 153(2), 163(1), 172(2), 190(1)\end{array}$ & $\mathrm{A} 13 \mathrm{a}^{75)}, \mathrm{A} 15 \mathrm{a}^{75)}, \mathrm{A} 19 \mathrm{a}$ \\
\hline${ }^{*}$ Oxypoda retunsa Assing, 2019 & $\begin{array}{l}53 a(1), 62(1), 65(1), 122(1), 125(1), \\
147(1), 149(1), 171(1), 214(4)\end{array}$ & A13a ${ }^{81)}, \mathrm{ABM} 19$ \\
\hline Oxypoda subnitida Mulsant \& Rey, 1875 & & A13a \\
\hline
\end{tabular}




\begin{tabular}{|c|c|c|}
\hline Species & Localities/samples & References \\
\hline Oxypoda vicina KRAATz, 1858 & 70(1), 96a(1), 192(1) & A15a \\
\hline Phloeopora corticalis (GRAVENHORST, 1802) & $74 \mathrm{a}(1), 150(3)$ & \\
\hline Phytosus balticus KraATZ, 1859 & & $\mathrm{~A} 15 \mathrm{a}, \mathrm{Br} 35^{58)}$ \\
\hline Phytosus spinifer CurTis, 1838 & 199(16) & App, Sa03 \\
\hline${ }^{*}$ Pronomaea wunderlei Assing, 2007 & 197(1) & A07c, Sz64 22) \\
\hline Stichoglossa graeca BernHAUER, 1905 & $74 \mathrm{a}(1)$ & \\
\hline Stichoglossa semirufa (ERICHSON, 1839) & & Kz58a, O87 \\
\hline${ }^{* * T e c t u s a}$ callicera Assing, 2002 & $182(5), 187(1)$ & A02, A15a \\
\hline${ }^{* *}$ Tectusa diktiana Assing, 2013 & & A13a, A15a \\
\hline${ }^{* *}$ Tectusa thriptica Assing, 2013 & & A13a \\
\hline${ }^{*}$ Typhlocyptus creticus Assing, 2019 & 13(1), 18(1), 66(1), 67(53) & $\mathrm{A} 18 \mathrm{c}^{73)}, \mathrm{ABM} 19$ \\
\hline Zoosetha graeca BERNHAUER, 1928 & $126(3), 127(5), 128(2)$ & \\
\hline Zyras haworthi (STEPHENS, 1832) & $\begin{array}{l}\text { 40(1), 44(1), 78(1), 80(1), 96(1), 172(1), } \\
\text { 173(1) }\end{array}$ & \\
\hline \multicolumn{3}{|l|}{ Scaphidinae } \\
\hline Scaphisoma agaricinum (LinnaEus, 1758) & $74 \mathrm{a}(1), 96 \mathrm{a}(1), 108 \mathrm{a}(1)$ & \\
\hline \multicolumn{3}{|l|}{ Osoriinae } \\
\hline${ }^{* *}$ Geomitopsis cretica Assing, 2019 & 106(3), 109(1) & ABM19 \\
\hline \multicolumn{3}{|l|}{ Oxytelinae } \\
\hline Anotylus clypeonitens (PANDELLÉ, 1867) & & $\begin{array}{l}\mathrm{C} 76, \mathrm{He} 84^{44)}, \mathrm{Ho63} 3^{44)} \\
\mathrm{Kz}^{48} \mathrm{~b}^{44)}, \mathrm{O} 87^{44)}, \mathrm{Sc} 12\end{array}$ \\
\hline Anotylus complanatus (ERICHSON, 1839) & 1(1), 30(1), 75a(444), 76(43) & A13a, C76, He84, O87, Sc09 \\
\hline Anotylus inustus (GRAVENHORST, 1806) & $\begin{array}{l}\text { 27(1), 35(1), 45(1), 59(3), 70(1), 71(1), } \\
73(1), 74(5), 75(1), 75 \mathrm{a}(32), 76(1), \\
79(2), 82(2), 83(1), 85(3), 90(3), 112(1), \\
123(1), 124(1), 125(1), 131(1), 144(1), \\
147(1), 149(2), 155(1), 162(1), 211(1)\end{array}$ & $\begin{array}{l}\text { A13a, A15a, Ho63, Kz58b, } \\
\text { O87, Sa03 }\end{array}$ \\
\hline Anotylus nitidulus (GRAVENHORsT, 1802) & $75 a(12), 219(1)$ & \\
\hline Anotylus sculpturatus (GRAVENHORST, 1806) & $\begin{array}{l}28(1), \quad 55(1), \quad 67(1), \quad 75 a(287), \quad 78(1), \\
151(1)\end{array}$ & A13a, A15a, O87 \\
\hline Anotylus tetracarinatus (BLOCK, 1799) & $75 \mathrm{a}(7)$ & Kz58b, Sz64 \\
\hline Bledius bicornis (GERMAR, 1823) & & $\mathrm{Sa03}$ \\
\hline Bledius frisius LoHSE, 1978 & & $\mathrm{Sa03}{ }^{45)}, \mathrm{Sc} 10$ \\
\hline Bledius graellsi FAUvEL, 1865 & $198(5)$ & \\
\hline Bledius minor Mulsant \& Rey, 1878 & 198(10), 257(4) & \\
\hline Carpelimus alutaceUs (FAUVEL, 1898) & & G15 \\
\hline Carpelimus bilineatus Stephens, 1834 & & G15 \\
\hline Carpelimus corticinus (GRAVENHORst, 1806) & $\begin{array}{l}40(1), \quad 88(8), \quad 89(5), \quad 90(15), \quad 94 \mathrm{a}(3), \\
166(1), 198(8), 199(1), 208(1)\end{array}$ & A13a, A15a, G15, O87, Sz64 \\
\hline Carpelimus despectus (BAUdi DI SELVE, 1870) & $89(1), 90(1)$ & \\
\hline $\begin{array}{l}\text { Carpelimus foveolatus foveolatus (SAHLBERG, } \\
\text { 1832) }\end{array}$ & & Ho63, Kz58b, O87 \\
\hline Carpelimus gracilis (MANNERHEIM, 1830) & & A13a \\
\hline Carpelimus insularis (KraATz, 1858) & $198(1)$ & App, G15 \\
\hline Carpelimus nitidus (BAUdi di SELVE, 1870) & $198(10)$ & \\
\hline
\end{tabular}




\begin{tabular}{|c|c|c|}
\hline Species & Localities/samples & References \\
\hline Carpelimus obesus (KIESENWETTER, 1844) & 219(1) & \\
\hline Carpelimus pusillus (GRAVENHORst, 1802) & $90(1)$ & G15 \\
\hline $\begin{array}{l}\text { Carpelimus transversicollis (SCHEERPELTZ, } \\
\text { 1947) }\end{array}$ & $198(2)$ & App \\
\hline Carpelimus troglodytes (ERICHSON, 1840) & & G15 \\
\hline Ochthephilus venustulus (RosenHauer, 1856) & $36 a(2), 90(1)$ & A13a, Ma14 \\
\hline Oxytelus laqueatus (MARsHAM, 1802) & & $\mathrm{Sa} 03$ \\
\hline Oxytelus sculptus GravenHorst, 1806 & & O87 \\
\hline Planeustomus cephalotes (ERICHSON, 1840) & & Kz57, O87 \\
\hline Planeustomus elegantulus (KRAATZ, 1857) & & Kz58b, O87 \\
\hline Platystethus alutaceus Tномsом, 1861 & & A16 \\
\hline Platystethus degener Mulsant \& REY, 1878 & $90(1), 219(2)$ & A15a, O87 ${ }^{43)}$ \\
\hline Platystethus nitens (C.R. SAHLBERG, 1832) & $90(1)$ & A15a \\
\hline Platystethus spinosus ERICHSON, 1840 & $88(1)$ & He84, Ho63, O87 \\
\hline Thinodromus bodemeyeri (BERNHAUER, 1902) & $90(1)$ & \\
\hline \multicolumn{3}{|l|}{ Steninae } \\
\hline Stenus aceris STEPHENS, 1833 & $\begin{array}{l}\text { 41a(2), 122(1), 141(1), 142(1), 147(2), } \\
\text { 150(1), 169(3), 177(1), 179(1), 180(1), } \\
\text { 191(1), 204(2) }\end{array}$ & A15a, Ho63, O87 \\
\hline${ }^{\star}$ Stenus ariadne Puthz, 1977 & & P77 \\
\hline Stenus assequens REy, 1884 & & App \\
\hline Stenus brunnipes lepidus WEISE, 1875 & $198(1)$ & Kz58b ${ }^{52)}, \mathrm{P} 68$ \\
\hline Stenus ganglbaueri BERNHAUER, 1905 & & App \\
\hline Stenus hospes ERICHSON, 1840 & $223(3)$ & $\mathrm{A} 13 \mathrm{a}^{10)}, \mathrm{A} 15 \mathrm{a}, \mathrm{Sa} 03$ \\
\hline Stenus languidus ERICHSON, 1840 & & O87, Ho63, Kz58b, P72a \\
\hline Stenus ochropus KIESENWETTER, 1858 & $\begin{array}{l}28(1), 32(3), 64(1), 108(1), 109 a(1) \\
109 b(1), 119(1), 135(8), 136(2), 159(1), \\
170(1), 171(2), 172(2), 174(3), 182(1), \\
203(7), 206(5)\end{array}$ & $\begin{array}{l}\text { A13a, A15a, O87, P71 } \\
\text { Sa03 }\end{array}$ \\
\hline Stenus ossium STEPHENs, 1833 & & P72b \\
\hline Stenus pallitarsis STEPHENs, 1833 & & App, Ho63, O87 \\
\hline Stenus parcior BERNHAUER, 1929 & & A13a, A15a, P08 \\
\hline Stenus picipes StePhens, 1833 & $197(32)$ & App \\
\hline Stenus subaeneus ERICHSON, 1840 & $\begin{array}{l}30(1), 133(3), 134(2), 135(1), 140(2), \\
141(1)\end{array}$ & A13a, A15a, O87, Kz58b \\
\hline Stenus turbulentus BondROIT, 1912 & $121(1), 122(2), 123(1), 129(1)$ & \\
\hline \multicolumn{3}{|l|}{ Eua esthetinae } \\
\hline Edaphus dissimilis (AuBÉ, 1863) & & Sz36 \\
\hline \multicolumn{3}{|l|}{ Scydmaeninae } \\
\hline${ }^{* * C e p h e n n i u m ~ a r c u a t u m ~ A s s i n g, ~} 2019$ & $24(1), 26(1)$ & ABM19 \\
\hline${ }^{* *}$ Cephennium chanianum Assing, 2019 & $\begin{array}{l}\text { 36(1), 43(1), 46(1), 49(1), 54(1), 149(1), } \\
168(1)\end{array}$ & ABM19 \\
\hline${ }^{* *}$ Cephennium curvatum Assing, 2019 & $2(1)$ & ABM19 \\
\hline${ }^{* *}$ Cephennium fortespinosum Assing, 2019 & 155(1), 106(3) & ABM19 \\
\hline${ }^{* *}$ Cephennium hamulatum Assing, 2019 & 13(1), 14(9), 125(4) & ABM19 \\
\hline
\end{tabular}




\begin{tabular}{|c|c|c|}
\hline Species & Localities/samples & References \\
\hline${ }^{* *}$ Cephennium idanum Assing, 2019 & $21(2), 105(1), 162(2)$ & ABM19 \\
\hline${ }^{* *}$ Cephennium latius Assing, 2019 & $105(1)$ & ABM19 \\
\hline${ }^{* *}$ Cephennium meybohmi Assing, 2019 & $168(1)$ & ABM19 \\
\hline${ }^{* *}$ Cephennium selenanum Assing, 2019 & $28(1), 84(1)$ & ABM19 \\
\hline${ }^{* *}$ Cephennium selinonum Assing, 2019 & $79(1)$ & ABM19 \\
\hline${ }^{* *}$ Cephennium sinuosum Assing, 2019 & 18(1), 20(7), 25(1) & ABM19 \\
\hline${ }^{* *}$ Cephennium thripticum Assing, 2019 & $6(1)$ & ABM19 \\
\hline Euconnus intrusus intrusus (SCHAUM, 1844) & & Kz58a, O87 \\
\hline${ }^{* *}$ Euconnus zakrius Меувонм, 2019 & 106(16), 155(14) & ABM19 \\
\hline Eutheia formicetorum REITTER, 1882 & $85(1), 148(1)$ & BB16 \\
\hline \#Eutheia paganettii Franz, 1971 & & BB16 ${ }^{66)}, \mathrm{Fz} 71$ \\
\hline Leptomastax bipunctata ReITteR, 1881 & & App \\
\hline${ }^{\star}$ Leptomastax cretica Мечвонм, 2019 & $\begin{array}{l}\text { 128(3), 135(2), 136(2), 136a(1), 167(1), } \\
\text { 171(1), 174(9) }\end{array}$ & $\begin{array}{l}\text { A13a }{ }^{71)}, \text { A15a }{ }^{71)}, \text { ABM19, } \\
\text { Ca96 }\end{array}$ \\
\hline${ }^{* *}$ Leptomastax thryptica Мечвонм, 2019 & & ABM19 \\
\hline Scydmaenus menozzii Franz, $1966^{65)}$ & & A13a \\
\hline${ }^{*}$ Scydmoraphes fuelscheri МЕчвонм, 2008 & $\begin{array}{l}28(1), \quad 54(1), \quad 73(1), \quad 130(1), \quad 144(1), \\
145(1), 149(3), 168(1)\end{array}$ & M08 \\
\hline *Scydmoraphes minotauri Мечвонм, 2008 & $128(1), 162(3)$ & A13a, M08 \\
\hline${ }^{*}$ Scydmoraphes ziegleri МЕҮвонм, 2008 & $\begin{array}{l}1(2), 8(1), 11(1), 15(2), 17(1), 61(1), \\
106(1), 155(3), 157(4), 159(1)\end{array}$ & ABM19, M08 \\
\hline ^Stenichnus aegialioides МеҮвонм, 2019 & $\begin{array}{l}44 \mathrm{a}(2), 102(1), 105(1), 115(1), 128(1) \\
147(1), 149(1), 151(1), 162(2), 167(3) \\
168(2), 175(1)\end{array}$ & $\mathrm{ABM} 19, \mathrm{BB} 16^{78)}, \mathrm{O} 87^{78)}$ \\
\hline $\begin{array}{l}\text { *Stenichnus basimpressus BLATTNÝ \& } \\
\text { BLATTNÝ, } 1916\end{array}$ & $\begin{array}{l}\text { 129(14), 135(1), 136a(1), 138(1), 138(4), } \\
161(2), 165(9), 167(1)\end{array}$ & BB16 \\
\hline ^Stenichnus brachati Мечвонм, 2019 & $\begin{array}{l}11(2), 110(1), 111(2), 119(3), 123(1) \\
128(2), 135(1), 136(1), 154(1), 157(2), \\
159(2), 161(7), 164(10), 170(1), 171(1)\end{array}$ & ABM19 \\
\hline Stenichnus creticus BlatTnÝ \& BLATTNÝ, 1916 & 112(5), 133(1), 134(1), 161(1) & A13a, BB16 \\
\hline $\begin{array}{l}\text { **Stenichnus hummleri BlatTNÝ \& BlatTNÝ, } \\
1916\end{array}$ & $92 \mathrm{~b}(1), 151(3), 152(2), 153(1)$ & BB16 \\
\hline${ }^{*}$ Stenichnus orientalis МЕувонм, 2019 & 155(3), 158(1) & ABM19 \\
\hline \multicolumn{3}{|l|}{ Leptotyphlinae } \\
\hline${ }^{* *}$ Allotyphlus candicus Assing, 2018 & 12(2), 16(2), 17(7), 18(1) & A18c \\
\hline${ }^{* *}$ Allotyphlus foedatus Assing, 2019 & $\begin{array}{l}34(26), 35(2), 36(22), 37(24), 38(6), \\
40(14), 46(1), 50(1), 51(1), 53(3), 54(8), \\
55(2), 57(2), 59(1), 67(2), 72(1), 74(1), \\
79(1), 81(1), 91(2), 92(1), 92 \mathrm{a}(12), \\
92 \mathrm{~b}(1), 95(1), 100(4)\end{array}$ & ABM19 \\
\hline${ }^{* * C r e t o t y p h l u s ~ c h a n i a n u s ~ A s s i n g, ~} 2019$ & $36(1), 100(2)$ & ABM19 \\
\hline${ }^{* *}$ Cretotyphlus hamatus Assing, 2019 & $9(2), 85(1), 119(1)$ & $\mathrm{A} 18 \mathrm{c}^{76)}, \mathrm{ABM} 19$ \\
\hline${ }^{* *}$ Cretotyphlus idanus Assing, 2019 & $105(3)$ & ABM19 \\
\hline **Kenotyphlus creticus Assing, 2018 & $25(7)$ & A18c \\
\hline
\end{tabular}




\begin{tabular}{|c|c|c|}
\hline Species & Localities/samples & References \\
\hline${ }^{* *}$ Kenotyphlus virgatus Assing, 2019 & $\begin{array}{l}40(2), 43(5), 46(18), 47(1), 69(2), 60(5) \\
73(1)\end{array}$ & ABM19 \\
\hline \multicolumn{3}{|l|}{ Pseudopsinae } \\
\hline Pseudopsis sulcata Newman, 1834 & & Z88 \\
\hline \multicolumn{3}{|l|}{ Paederinae } \\
\hline Achenium depressum (GRAVENHORST, 1802) & $120(1)$ & A10b, O87 ${ }^{39)}$, Sz64 \\
\hline Astenus lyonessius (Joy, 1908) & $139(1)$ & A13a, A15a, O87 \\
\hline${ }^{* *}$ Astenus minos Assing, 2003 & $31(2), 33(1)$ & A03a, A15a \\
\hline Astenus procerus (GRAVENHORST, 1806) & & A13a, A15a, O87, Sz64 \\
\hline Astenus thoracicus (BAUDI DI SELVE, 1857) & $\begin{array}{l}63(1), \quad 71(2), \quad 95 a(2), \quad 120(4), \quad 159(9) \\
161(1), 163(2), 181(1), 220(3), 221(2)\end{array}$ & A13a, A15a, O87 \\
\hline${ }^{* *}$ Astenus thripticus Assing, 2013 & & A13a, A15a \\
\hline Domene stilicina (ERICHSON, 1840) & $71(1)$ & $\begin{array}{l}\text { A13a, A15a, App, Bo77 }{ }^{31)} \\
\text { C76, Kz58a, O87, Sz64 }\end{array}$ \\
\hline Hypomedon debilicornis (Wollaston, 1857) & $76(1)$ & \\
\hline${ }^{*}$ Leptobium creticum CoIfFAIT, 1973 & $\begin{array}{l}\text { 15(1), 21(2), 24(2), 26(1), 28(3), 34(1), } \\
37(1), 71(1), 101(1), 103(1), 105(4), \\
119(2), 120(1), 125(1), 126(1), 127(1) \\
154(1), 160(2), 162(3), 164(1), 171(1) \\
179(1), 203(1), 204(1), 215(2), 220(3), \\
221(1)\end{array}$ & $\begin{array}{l}\text { A05a, A09a, A09b, A13a, } \\
\text { A15a, A17a, Bo84 }{ }^{30)}, \text { O }^{38} \\
\text { C73, Sz64 }\end{array}$ \\
\hline Leptobium gracile (GRAVENHORST, 1802) & & $\begin{array}{l}\text { A05a, A09a, He84, Kz58a, } \\
\text { O87, Sz64 }\end{array}$ \\
\hline${ }^{* *}$ Leptobium thryptisense Assing, 2005 & 4(1), 107(1), 123(1), 129(1), 179(1) & A05a, A13a, App \\
\hline Lithocharis ochracea (GRAVENHORST, 1802) & $76(3)$ & \\
\hline${ }^{*}$ Lobrathium candicum BoRdonI, 2009 & & $\begin{array}{l}\text { A07b }{ }^{21)}, A 12, A 14 a, A 15 a, \\
\text { Bo09, C76 }{ }^{21)}\end{array}$ \\
\hline Luzea graeca KraATZ, 1857 & & A10a \\
\hline${ }^{*}$ Medon beroni CoIfFAIT, 1970 & $\begin{array}{l}18 \mathrm{a}(1), \quad 39(1), 41 \mathrm{a}(22), 60(1), \quad 68(1), \\
93(12), 100(2), 144(6), 149(10), 154(4), \\
156(1), 184(2)\end{array}$ & $\mathrm{A} 04 \mathrm{a}, \mathrm{C} 70, \mathrm{C} 76, \mathrm{O} 87^{40)}$ \\
\hline Medon brunneus (ERICHSON, 1839) & & A04a, Sz64 \\
\hline${ }^{*}$ Medon cerrutii CoIfFAIT, 1976 & 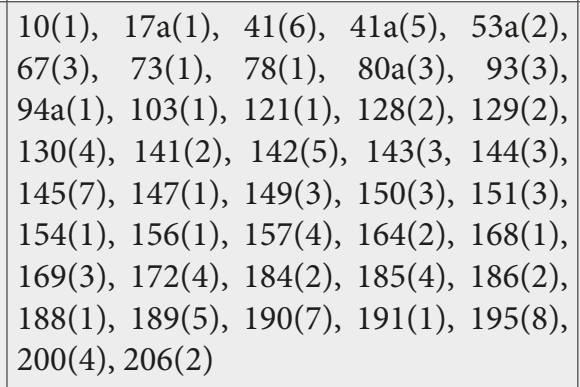 & A04a, C76 \\
\hline Medon dilutus pythonissa (SAULCY, 1865) & $\begin{array}{l}5(1), 18(2), 21(3), 32(5), 41(1), 61(1), \\
67(3), 80(1), 93(1), 105(1), 119(1), \\
121(1), 122(1), 123(1), 129(2), 141(1), \\
142(2), 143(1), 144(2), 145(2), 149(1), \\
150(3), 162(2), 164(1), 174(1), 183(1), \\
190(1), 191(2), 192(2), 193(1), 195(3), \\
206(14), 207(7), 213(22), 220(1)\end{array}$ & $\begin{array}{l}\text { A04a, A07e, A13a, A13c, } \\
\text { A15a, A18b }\end{array}$ \\
\hline Medon maronitus (SAULCY, 1865) & $93(1), 154(1)$ & \\
\hline
\end{tabular}




\begin{tabular}{|c|c|c|}
\hline Species & Localities/samples & References \\
\hline Medon pocofer (PEYRon, 1858) & & O87 \\
\hline Medon rufiventris (NoRDMANN, 1837) & $108 \mathrm{a}(1)$ & A04a, A18b \\
\hline Micranops pilicornis (SAULCY, 1870) & & Fr97, Fr10 \\
\hline Micrillus testaceus (ERICHSON, 1840) & & A08a \\
\hline Ochthephilum turkestanicum (KoRGE, 1968) & & $\mathrm{A} 09 \mathrm{c}, \mathrm{A} 15 \mathrm{a}^{48)}$ \\
\hline Paederus fuscipes fuscipes (CurTIs, 1826) & $166(1), 219(3)$ & A15a, Kz58a ${ }^{56)}$, O87, Sz64 \\
\hline Paederus littoralis GRAVENHORST, $1802^{27}$ & & App, Sz64 \\
\hline Platydomene picipes picipes (ERICHSON, 1840) & $90(1)$ & A13a, Kz58a, O87 \\
\hline${ }^{\star}$ Pseudobium creticum Assing, 2019 & $90(8)$ & $\mathrm{A} 15 \mathrm{a}^{79)}, \mathrm{ABM} 19$ \\
\hline ^Pseudolathra quadricollis (FAUVEL, 1875) & $94(3), 166(1)$ & Bo86 ${ }^{83)}$ \\
\hline Pseudomedon obscurellus (ERICHSON, 1840) & 91(1), 94(14) & A08d, A09d, Sz64 \\
\hline Rugilus orbiculatus (PAYKULL, 1789) & $77(15), 223(1)$ & O87, Sa03, Sz64 \\
\hline Scopaeus creticus FRISCH, 1994 & $90(2), 171(2)$ & Fr94, Fr99b \\
\hline Scopaeus debilis Носннuтн, 1851 & & Fr99a \\
\hline Scopaeus gracilis (SPERK, 1835) & $90(3)$ & Fr98 \\
\hline${ }^{*}$ Scopaeus muehlei FrIsCH, 1994 & $94 \mathrm{a}(4)$ & A13a, Fr94 \\
\hline${ }^{*}$ Sunius diktianus Assing, 2013 & $24(1), 112(1)$ & A13a \\
\hline Sunius fallax (LoKAy, 1919) & $\begin{array}{l}\text { 3(1), 71(5), 87(1), 94a(2), 111(1), 112(9), } \\
\text { 112(3), 120(3), 136(1), 166(4), 167(1), } \\
\text { 171(3), 175(2), 201(10), 222(7), 223(1) }\end{array}$ & $\begin{array}{l}\text { A08c, A08e, A13a, A15a, } \\
\text { A15b, He84 }{ }^{41)}, \text { O } 87^{41)}\end{array}$ \\
\hline 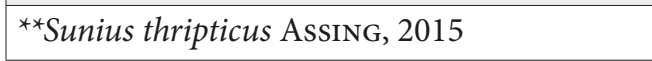 & $19(1), 131(1)$ & A15a \\
\hline $\begin{array}{l}\text { Throbalium dividuum dividuum (ERICHSON, } \\
\text { 1840) }\end{array}$ & & Kz58a, O87 \\
\hline \multicolumn{3}{|l|}{ Staphylininae } \\
\hline Bisnius cephalotes (GRAVENHORST, 1802) & & O87 \\
\hline Bisnius sordidus (GRAVENHORST, 1802) & 76(22), 211(1), 216(1), 217(1) & O87, Sz64 \\
\hline Cafius xantholoma (GRAVENHORST, 1806) & $257 \mathrm{a}(1)$ & $\mathrm{Sa} 03$ \\
\hline Erichsonius cinerascens (GRAVENHORST, 1802) & $197(1)$ & \\
\hline${ }^{*}$ Gabrius candicus Assing, 2019 & $94 \mathrm{a}(18), 166(8)$ & ABM19 \\
\hline Gabrius nigritulus (GRAVENHORST, 1802) & 194(1), 200(2), 219(2) & A13a, He84, O87, Sz64 \\
\hline Gabronthus maritimus (MoTsCHULSKy, 1858) & $75 a(1), 76(83), 222(1)$ & \\
\hline Gauropterus fulgidus (FABRICIUs, 1787) & & He84, Kz58a, O87, Sz64 \\
\hline Gauropterus sanguinipennis (KoLENATI, 1846) & & Kz57, Kz58a, O87 \\
\hline Gyrohypnus angustatus STEPHENs, 1833 & 41a(14), 42(1), 93(1), 154(1) & A03c, A07a, A15a \\
\hline Gyrohypnus fracticornis (MüLLER, 1776) & & App, $\mathrm{O} 87^{49)}, \mathrm{Sz} 64$ \\
\hline Gyrohypnus punctulatus (PAYKULL, 1789) & $41 \mathrm{a}(1)$ & \\
\hline Heterothops dissimilis (GRAVENHORST, 1802) & $94 \mathrm{a}(1), 219(3), 222(2), 223(3)$ & $\mathrm{A} 13 \mathrm{a}^{72)}, \mathrm{A} 15 \mathrm{a}^{72)}$ \\
\hline Leptacinus batychrus (GyLlenHAL, 1827) & $76(1)$ & $\mathrm{He} 84, \mathrm{O} 87$ \\
\hline Leptacinus intermedius DoNISTHORPE, 1936 & $76(2)$ & \\
\hline Megalinus flavocinctus (Носннштн, 1849) & & $\begin{array}{l}\text { A07a, Bo76, Bo07, Bo14, } \\
\text { Kz58a }^{13)}, \mathrm{O}^{13} 7^{13)}, \mathrm{Sz}^{13)}\end{array}$ \\
\hline $\begin{array}{l}\text { Neobisnius lathrobioides (BAUDI DI SELVE, } \\
\text { 1848) }\end{array}$ & $90(1), 200(1)$ & O88 ${ }^{37)}, \mathrm{Sz}_{64}{ }^{16)}$ \\
\hline Neobisnius procerulus (GRAVENHORST, 1806) & $94 \mathrm{a}(1)$ & \\
\hline
\end{tabular}




\begin{tabular}{|c|c|c|}
\hline Species & Localities/samples & References \\
\hline Ocypus mus (BRULLÉ, 1832) & $216(1)$ & A13a, A15a, Ho65, O87 \\
\hline Ocypus olens (O. MüLLER, 1764) & & $\begin{array}{l}\text { A13a, A15a, He84, Ho65, } \\
\text { Kz58a, O87 }\end{array}$ \\
\hline Ocypus sericeicollis (MÉNÉTRIÉs, 1832) & $225(1), 227(3)$ & $A 13 a^{6)}, A 5$ \\
\hline Ocypus simulator (EPPELSHEIM, 1878) & & $\mathrm{Sa} 03$ \\
\hline Othius laeviusculus StePHens, 1833 & $111(1), 120(2), 179(1)$ & $\begin{array}{l}\text { A03b, A08f, A10c, A13a, } \\
\text { A13b, A15a, C76, Ho58, } \\
\text { O87, Sz64 }\end{array}$ \\
\hline $\begin{array}{l}\text { Othius lapidicola Märkel \& KIESENWETTER, } \\
1848\end{array}$ & 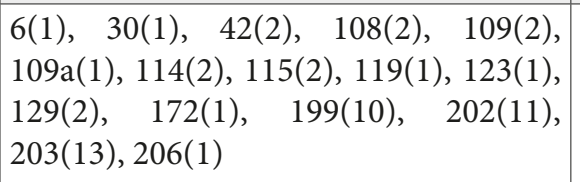 & $\begin{array}{l}\text { A97b, A03b, A08f, A13a, } \\
\text { A13b, A15a }\end{array}$ \\
\hline $\begin{array}{l}\text { Phacophallus parumpunctatus (GyllenHAL, } \\
\text { 1827) }\end{array}$ & $75 a(1)$ & \\
\hline Philonthus concinnus (GRAVENHORST, 1802) & $76(2)$ & A15a, O87, Sz64 \\
\hline Philonthus corruscus (GravenHoRst, 1802) & $224(1)$ & \\
\hline Philonthus debilis (GravenHorst, 1802) & $76(1)$ & \\
\hline Philonthus discoideus (Gravenhorst, 1802) & & Sz64 \\
\hline Philonthus ebeninus (GravenHorst, 1802) & & Kz58a \\
\hline Philonthus heterodoxus Mulsant \& REY, 1876 & $256(1)$ & \\
\hline Philonthus intermedius (LACORDAIRE, 1835) & & A15a, Kz58a, O87, Sz64 \\
\hline Philonthus longicornis (PAYKULL, 1800) & $76(2)$ & O87 \\
\hline Philonthus nitidicollis (LACORDAIRE, 1835) & $71(1)$ & A13a, A15a, O87 ${ }^{17)}, S_{264}{ }^{17)}$ \\
\hline Philonthus parvicornis (GRAVENHORST, 1802) & & Kz58a $\mathrm{a}^{51)}$ \\
\hline Philonthus quisquiliarius (GyllenHAL, 1810) & $89(1), 198(4), 219(2), 223(2)$ & A13a \\
\hline Philonthus rufimanus HeER, 1839 & $219(2)$ & $\begin{array}{l}\text { A13a, Ho65, Kz58a, O87, } \\
\text { Sa03, Sz64 }\end{array}$ \\
\hline Philonthus splendens (FABRICIUs, 1792) & & Kz58a, O87 \\
\hline Philonthus varians (PAYKuLL, 1789) & $76(9), 220(1)$ & O87 \\
\hline Philonthus ventralis (GRAVENHORST, 1802) & $76(1)$ & \\
\hline Quedius abietum KIESENWETtER, 1858 & & A07f, O87, Sz64 \\
\hline Quedius cinctus (PAYKulL, 1790) & $68(1), 75 a(4)$ & $\begin{array}{l}\text { A15a, C76, Ly21, Mn35, } \\
\text { O87, Sz64 }\end{array}$ \\
\hline Quedius cruentus (Olivier, 1795) & $225(1), 226(1)$ & Ly13, Mn35 \\
\hline Quedius fulgidus (FABRICIUs, 1793) & & Mn35 \\
\hline Quedius humeralis STEPHENs, 1832 & $160(1), 179(1)$ & A13a, A15a, C76 ${ }^{28)}$, Sz64 \\
\hline Quedius levicollis (BRULLÉ, 1832) & $214(1)$ & 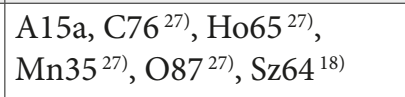 \\
\hline Quedius nemoralis BAUdI DI SELVE, 1848 & $\begin{array}{l}\text { 41a(1), 73(1), } 74(1), \quad 82(1), \quad 83(1), \\
108(3), 109 \mathrm{a}(2), 114(1), 118(1), 119(6), \\
123(3), 126(2), 128(1), 131(1), 133(3), \\
144(1), 147(1), 153(1), 157(1), 158(1), \\
165(1), 169(1), 171(1), 184(1), 193(2), \\
195(3), 201(10), 202(3), 203(4), 204(2), \\
\text { 206(2), 209(1), 214(1) }\end{array}$ & A13a, A15a, C76 ${ }^{11)}$ \\
\hline${ }^{*}$ Quedius praecisus Assing, 2015 & $62(2)$ & A15a \\
\hline Quedius scintillans (GRAVENHORST, 1806) & $41 \mathrm{a}(1), 76(16)$ & A15a, C76, Sz64 \\
\hline
\end{tabular}




\begin{tabular}{|c|c|c|}
\hline Species & Localities/samples & References \\
\hline Quedius semiaeneus (STEPHENs, 1833) & & Sz64 \\
\hline Quedius semiobscurus (MARsham, 1802) & $41 \mathrm{a}(1)$ & A13a, Sz64 ${ }^{19)}$ \\
\hline${ }^{*}$ Quedius sigwalti CoIfFAIT, 1972 & $\begin{array}{l}18 \mathrm{a}(2), \quad 41 \mathrm{a}(4), \quad 42(1), \quad 67(1), \quad 68(2), \\
74 \mathrm{a}(2), 110(1), 150(1), 153(1), 195(2) \\
197(13), 200(2), 215(1), 223(5)\end{array}$ & A13a ${ }^{5)}, A 15 a, C 72$ \\
\hline Remus filum (KIESENWETTER, 1849) & 199(3), 257a(1) & $\mathrm{Sa} 03$ \\
\hline Remus pruinosus (ERICHSON, 1840) & 199(10) & $\mathrm{Sa} 03$ \\
\hline Remus sericeus Holme, 1837 & $257 \mathrm{a}(1)$ & $\mathrm{Sa} 03$ \\
\hline Stenistoderus cephalotes (KrAATZ, 1858) & $94(1)$ & A07a \\
\hline Stenistoderus nothus (ERICHSON, 1839) & & $\mathrm{He} 84, \mathrm{O} 87$ \\
\hline${ }^{*}$ Xantholinus creticus Assing, 2006 & $66(1), 94(1)$ & A06, A07a, A17b \\
\hline${ }^{*}$ Xantholinus erinaceus Assing, 2015 & & $\begin{array}{l}\mathrm{A} 07 \mathrm{a}^{20)}, \mathrm{A} 15 \mathrm{a}, \mathrm{Bo} 07^{32)} \\
\mathrm{Bo}^{32)}, \mathrm{Bo} 17^{32)}, \mathrm{Kz}^{32} \mathrm{a}^{32)} \\
\mathrm{O} 87^{32)}, \mathrm{Sz} 64^{14)}\end{array}$ \\
\hline${ }^{*}$ Xantholinus minos Assing, 2008 & 39(1), 41a(1), 76(2) & 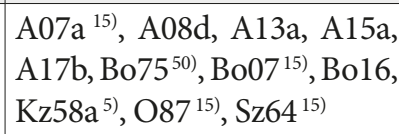 \\
\hline
\end{tabular}

\section{Localities/samples:}

December-January 2017/2018, leg. Assing: 1: ca 20 km E Agios Nikolaos, SW Lastros, 3508'14"N, 2553'53"E, 340 m, (temporary) stream valley with Quercus ilex, Q. sp., olive trees, etc., soil washing, 24.XII.2017; 2: ca $20 \mathrm{~km}$ E Agios

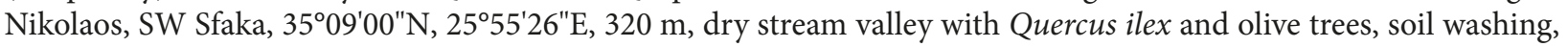

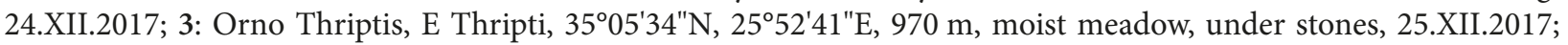
4: Orno Thriptis, near peak, 35 $04^{\prime} 54^{\prime \prime} \mathrm{N}, 25^{\circ} 52^{\prime} 49^{\prime \prime} \mathrm{E}, 1270 \mathrm{~m}$, phrygana, litter, moss, and soil sifted, 25.XII.2017;

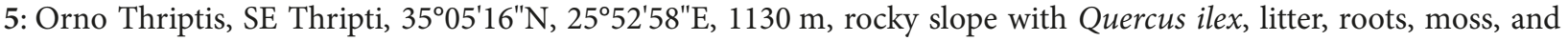
soil sifted, 25.XII.2017; 6: same data as 5, but soil washing; 7: $10 \mathrm{~km}$ WNW Agios Nikolaos, 35¹3'12"N, 25³6'12"E, $390 \mathrm{~m}$, dry stream valley with Quercus ilex, soil washing, 26.XII.2017; 8: $11 \mathrm{~km}$ WNW Agios Nikolaos, 35²12'56"N, $25^{\circ} 35^{\prime} 51^{\prime E} \mathrm{E}, 520 \mathrm{~m}$, margin of Quercus ilex forest, soil washing, 26.XII.2017; 9: NE Lassithi Plateau, 35 $5^{\circ} 12^{\prime} 45^{\prime \prime} \mathrm{N}$, $25^{\circ} 31^{\prime} 53^{\prime \prime E}, 850 \mathrm{~m}$, dry ruderal stream valley with old Platanus, soil washing, 26.XII.2017; 10: NE Lassithi Plateau, $35^{\circ} 11^{\prime} 00^{\prime \prime} \mathrm{N}, 25^{\circ} 30^{\prime} 53^{\prime \prime E}, 860 \mathrm{~m}$, Quercus ilex forest, soil washing, 26.XII.2017; 11: W Kritsa, road to Katharo Plateau, $35^{\circ} 09^{\prime} 41^{\prime \prime} \mathrm{N}, 25^{\circ} 38^{\prime} 21^{\prime \prime} \mathrm{E}, 450 \mathrm{~m}$, dry stream valley with Quercus ilex, gravelly soil, soil washing; 12: W Kritsa, road to

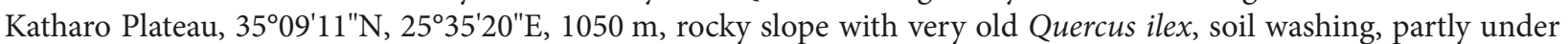
snow, 27.XII.2017; 13: S Kritsa, 350' $47^{\prime \prime} \mathrm{N}, 25^{\circ} 38^{\prime} 24^{\prime \prime E}, 400 \mathrm{~m}$, dry stream valley with Quercus ilex, soil washing,

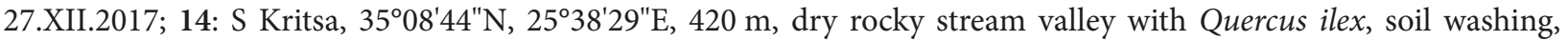

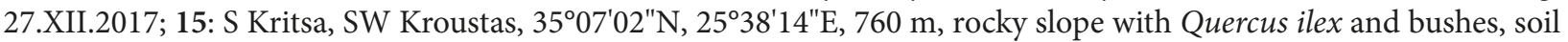

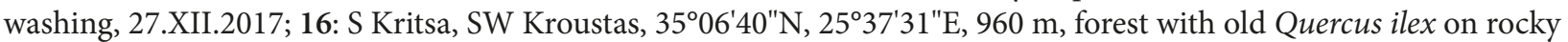

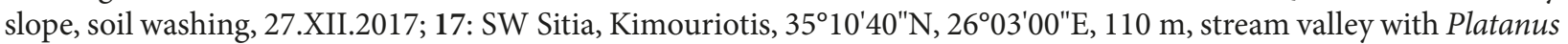
and rich undergrowth, soil washing, 28.XII.2017; 17a: same data as 17, but litter sifted; 18: SW Sitia, NW Makrigialos, $35^{\circ} 03^{\prime} 31^{\prime \prime} \mathrm{N}, 25^{\circ} 56^{\prime} 49^{\prime \prime} \mathrm{E}, 70 \mathrm{~m}$, valley with small temporary stream, with Platanus, bushes, undergrowth, and reed, soil washing, 30.XII.2017; 18a: same data as 18, but moist Platanus litter near small temporary stream sifted; 19: SW Sitia, N Stavrochori, $35^{\circ} 05^{\prime} 13^{\prime \prime} \mathrm{N}, 25^{\circ} 56^{\prime} 45^{\prime} \mathrm{E}, 280 \mathrm{~m}$, dry stream valley with Platanus, olive trees, and rich

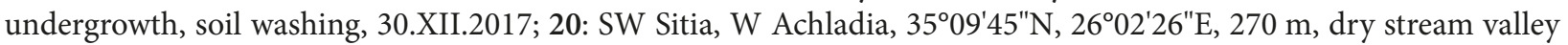
with old oak, other deciduous trees, and undergrowth, soil washing, 30.XII.2017; 21: Psiloritis, NE Livadia, 35¹8'56"N, 24 $49^{\prime} 07^{\prime E}$, $440 \mathrm{~m}$, stream valley with Platanus, Quercus sp., and undergrowth, soil washing, 31.XII.2017; 22: Psiloritis, NE Livadia, $35^{\circ} 18^{\prime} 52^{\prime \prime} \mathrm{N}, 24^{\circ} 48^{\prime} 44^{\prime \prime} \mathrm{E}, 470 \mathrm{~m}$, NW slope with Quercus ilex, other deciduous trees, and bushes, soil washing, 31.XII.2017; 23: Psiloritis, SE Garazo, Omala, 35²0'11"N, 2447'42"E, $290 \mathrm{~m}$, dry ruderal stream valley, soil

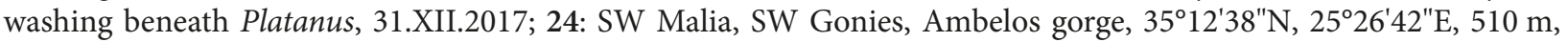

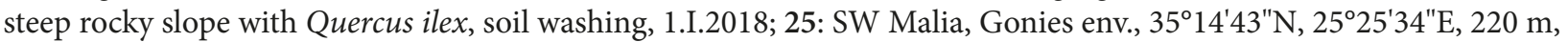
arable land and pastures, spot with very old Quercus sp. with undergrowth, soil washing, 1.I.2018; 26: SW Malia,

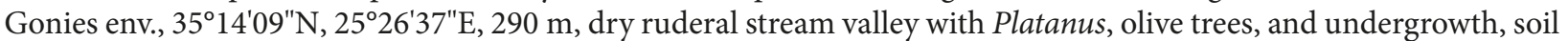

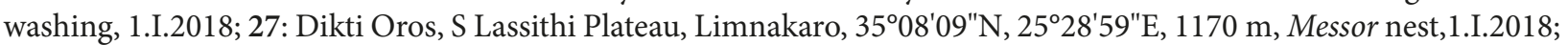
28: SE Malia, Selena Oros, $35^{\circ} 14^{\prime} 40^{\prime \prime} \mathrm{N}, 25^{\circ} 31^{\prime} 03^{\prime \prime E}, 880 \mathrm{~m}$, calcareous N-slope with Quercus ilex, litter, roots, and moss sifted, 2.I.2018; 29: SE Malia, Selena Oros, 35¹5'24"N, 2532'04"E, 700 m, calcareous plateau, Messor nest, 2.I.2018. 
March 2018, leg. Assing: 30: Lefka Ori, Kallergi Refuge, $35^{\circ} 19^{\prime} 19^{\prime \prime} \mathrm{N}, 23^{\circ} 55^{\prime} 46^{\prime \prime E}, 1600 \mathrm{~m}$, litter and grass under shrubs

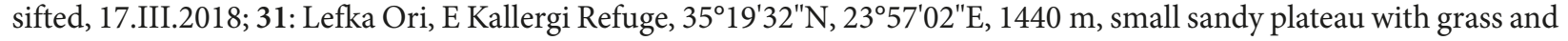
stones, under stones, 17.III.2018; 32: Lefka Ori, E Kallergi Refuge, 35¹9'38"N, 2357'10"E, 1450 m, N-slope with phrygana, bushes, shrubs, and fern, litter and grass beneath bushes and shrubs sifted, 17.III.2018; 33: Lefka Ori, NE Kallergi Refuge $35^{\circ} 19^{\prime} 45^{\prime \prime} \mathrm{N}, 23^{\circ} 57^{\prime} 08^{\prime \prime} \mathrm{E}, 1410 \mathrm{~m}$, grassy track margin, under stones, 17.III.2018; 34: SW Chania,

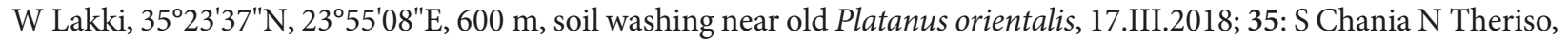
$35^{\circ} 24^{\prime} 52^{\prime \prime} \mathrm{N}, 23^{\circ} 59^{\prime} 14^{\prime} \mathrm{E}, 610 \mathrm{~m}$, rocky stream valley, soil washing near old Platanus orientalis on slope, 17.III.2018;

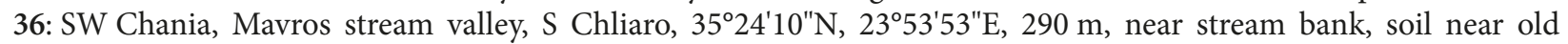
Platanus orientalis sifted, 18.III.2018; 36a: same data, but stream bank, gravel floated; 37: SW Chania, Mavros stream valley, SW Chliaro, 352 $23^{\prime} 42^{\prime \prime N}, 23^{\circ} 54^{\prime} 04^{\prime \prime E}, 400 \mathrm{~m}$, slope with old chestnut and Rubus, soil floated, 18.III.2018; 38: SW Chania, Mavros stream valley, SW Chliaro, 352 $23^{\prime} 27^{\prime \prime} \mathrm{N}, 23^{\circ} 54^{\prime} 07^{\prime \prime} \mathrm{E}, 470 \mathrm{~m}$, soil washing near Platanus orientalis, 18.III.2018; 39: SW Chania, Mavros stream valley, SW Chliaro, 35²3'59"N, 2353'59"E, 320 m, litter near stream bank sifted, 18.III.2018; 40: SW Chania, Mavros stream valley, SW Chliaro, 35²3'56"N, 235' $46^{\prime \prime}$ E, 380 m, slope with cherry orchard and very old chestnut, soil washing, 18.III.2018; 41: SW Chania, Mavros stream valley, $35^{\circ} 23^{\prime} 48^{\prime \prime} \mathrm{N}$, $23^{\circ} 53^{\prime} 40^{\prime \prime} \mathrm{E}, 410 \mathrm{~m}$, dark stream valley with old chestnut, stony soil washed, 18.III.2018; 41a: same data, but deep moist

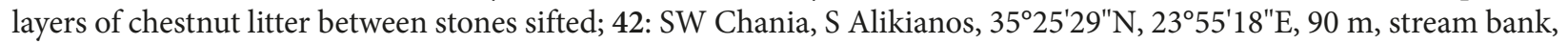

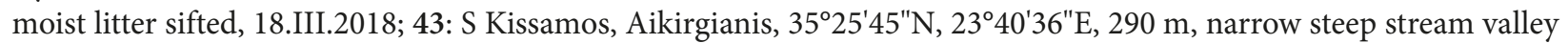
with Platanus orientalis, soil washing near stream, 19.III.2018; 44: S Kissamos, NE Sirikari, 35²4'48"N, $23^{\circ} 39^{\prime} 04^{\prime \prime} \mathrm{E}$, $500 \mathrm{~m}$, moist slope with very old chestnut, ferns, etc., soil washing, 19.III.2018; 44a: same data, but dead chestnut tree

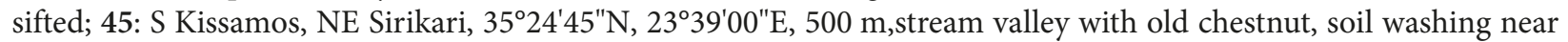

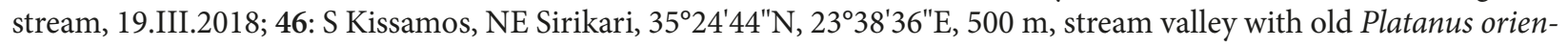
talis, soil washing near stream, 19.III.2018; 47: S Kissamos, W Sirikari, 35²4'28"N, 233' $37^{\prime \prime} \mathrm{E}, 470$ m, old Platanus

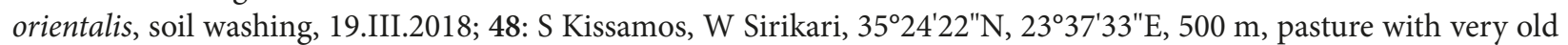

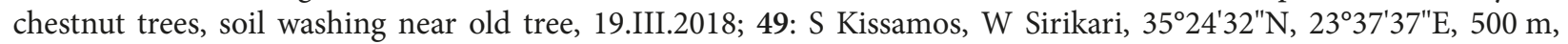
pasture with old chestnut trees, soil washing near tree, 19.III.2018; 50: SW Chania, WSW Chliaro, 35²4'26"N, $23^{\circ} 53^{\prime} 15^{\prime \prime} \mathrm{E}, 210 \mathrm{~m}$, forest margin, soil washing beneath very old chestnut tree, 20.III.2018; 51: SW Chania, W Prases, $35^{\circ} 22^{\prime} 33^{\prime \prime} \mathrm{N}, 23^{\circ} 49^{\prime} 25^{\prime \prime} \mathrm{E}, 650 \mathrm{~m}$, pasture with old chestnut and Platanus orientalis, soil washing, 20.III.2018; 52: SW Chania, SW Prases, $35^{\circ} 21^{\prime} 45^{\prime \prime} \mathrm{N}, 23^{\circ} 48^{\prime} 59^{\prime \prime} \mathrm{E}, 800 \mathrm{~m}$, slope with very old Platanus orientalis, soil washing under

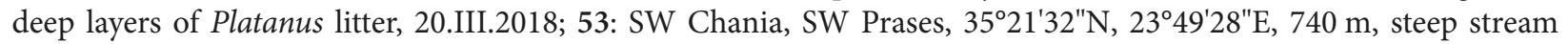
valley with very old Platanus orientalis, soil washing, 20.III.2018; 53a: same data, but litter sifted; 54: SW Chania, SW Prases, $35^{\circ} 21^{\prime} 43^{\prime \prime} \mathrm{N}, 23^{\circ} 49^{\prime} 24^{\prime \prime} \mathrm{E}, 640 \mathrm{~m}$, steep stream valley with old Platanus orientalis, soil washing, 20.III.2018; 55: SW Chania, SW Prases, $35^{\circ} 21^{\prime} 43^{\prime \prime} \mathrm{N}, 23^{\circ} 49^{\prime} 28^{\prime \prime} \mathrm{E}, 650 \mathrm{~m}$, margin of pasture, soil washing near old Platanus orientalis, 20.III.2018; 56: S Kissamos, N Archontico, 3520'18"N, 2339'52"E, 760 m, pasture,under stones, 21.III.2018;

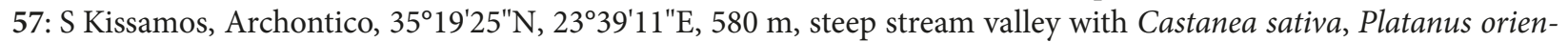
talis, and Quercus ilex, soil washing, 21.III.2018; 58: S Kissamos, Archontico, 35 $18^{\prime} 55^{\prime \prime} \mathrm{N}, 23^{\circ} 38^{\prime} 47^{\prime \prime} \mathrm{E} 530 \mathrm{~m}$, beaten

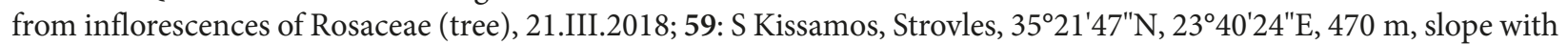

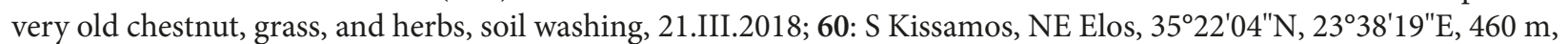
stream valley, soil washing near old Platanus and Castanea (relatively dry soil), 21.III.2018; 61: S Kissamos, Elos, $35^{\circ} 21^{\prime} 49^{\prime \prime} \mathrm{N}, 23^{\circ} 38^{\prime} 18^{\prime} \mathrm{E}, 500 \mathrm{~m}$, ruderal habitat with very old chestnut, herbs, and fern, soil washing, 21.III.2018; 62: Lefka Ori, E Kallergi Refuge, $35^{\circ} 19^{\prime} 38^{\prime \prime} \mathrm{N}, 23^{\circ} 57^{\prime} 02^{\prime \prime} \mathrm{E}, 1460 \mathrm{~m}$, slope with phrygana and bushes, litter and grass roots sifted, 22.III.2018; 63: Lefka Ori, Kallergi Refuge, $35^{\circ} 19^{\prime} 22^{\prime \prime} \mathrm{N}, 2^{\circ} 55^{\prime} 53^{\prime \prime} \mathrm{E}, 1560 \mathrm{~m}$, sandy plateau with grass,

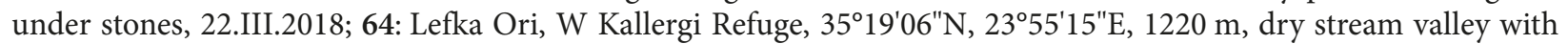
Acer, litter and roots of grass and herbs sifted, 22.III.2018; 65: SW Chania, SW Prases, 35²21'30"N, $23^{\circ} 49^{\prime} 40^{\prime \prime} \mathrm{E}, 820 \mathrm{~m}$, slope with Platanus, other trees, and fern, litter and roots sifted, 22.III.2018; 66: SW Chania, S Nteres, 35 $24^{\prime} 00^{\prime \prime} \mathrm{N}$, 2351'00"E $360 \mathrm{~m}$, stream valley with old chestnut and undergrowth (fern, herbs), soil washing, 22.III.2018; 67: SW Chania, S Nteres, $35^{\circ} 24^{\prime} 15^{\prime \prime} \mathrm{N}, 23^{\circ} 50^{\prime} 58^{\prime \prime} \mathrm{E}, 330 \mathrm{~m}$, slope with very old chestnut, fern, and herbs, soil washing, 22.III.2018; 68: SW Chania, S Nteres, $35^{\circ} 24^{\prime} 12^{\prime \prime} \mathrm{N}, 23^{\circ} 50^{\prime} 58^{\prime \prime} \mathrm{E}, 350 \mathrm{~m}$, bottom of vertical road margin with trickling

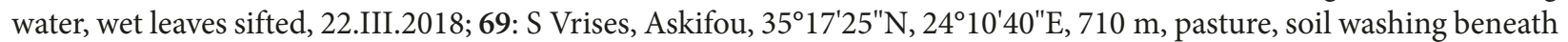

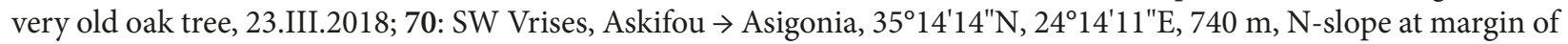
pasture, soil washing between rocks near old Quercus ilex, 23.III.2018; 71: SW Asigonia, Kallikratis, 35⒈ $14^{\prime} 18^{\prime \prime} \mathrm{N}$, $24^{\circ} 15^{\prime} 44^{\prime \prime} \mathrm{E}, 720 \mathrm{~m}$, margin of arable land, under stones, 23.III.2018; 72: S Asigonia, E Kallikratis, $35^{\circ} 14^{\prime} 58^{\prime \prime} \mathrm{N}, 24^{\circ} 16^{\prime} 38^{\prime \prime} \mathrm{E}$, $770 \mathrm{~m}$, margin of pasture with fern, soil washing soil washing near old Platanus orientalis, 23.III.2018; 73: Asigonia, $35^{\circ} 16^{\prime} 08^{\prime \prime} \mathrm{N}, 24^{\circ} 16^{\prime} 55^{\prime \prime} \mathrm{E}, 380 \mathrm{~m}$, steep road margin, soil washing near old oak tree, 23.III.2018; 74: SW Chania, S Nteres, $35^{\circ} 24^{\prime} 48^{\prime \prime} \mathrm{N}, 23^{\circ} 50^{\prime} 46^{\prime} \mathrm{E}, 280 \mathrm{~m}$, steep stream valley with very old chestnut trees, soil washing, 24.III.2018; 74a: same data, but under bark of dead chestnut trees; 75: SW Chania, N Nteres, $35^{\circ} 27^{\prime} 40^{\prime \prime} \mathrm{N}, 23^{\circ} 50^{\prime} 19^{\prime \prime} \mathrm{E}, 170 \mathrm{~m}$, stream valley with old chestnut trees and fern undergrowth, soil washing, 24.III.2018; 75a: same data, but road margin, horse dung

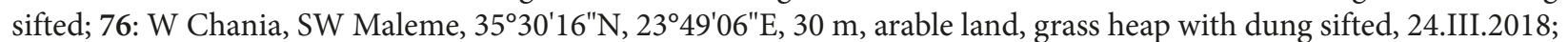

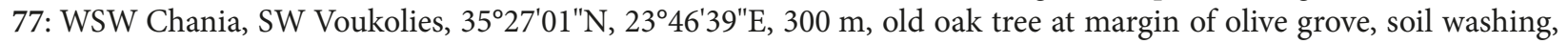




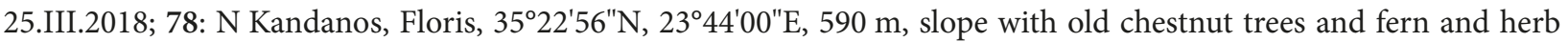

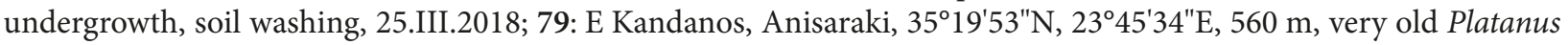
orientalis at margin of olive grove, ruderal habitat below house, soil washing, 25.III.2018; 80: SE Kandanos, $35^{\circ} 19^{\prime} 18^{\prime \prime} \mathrm{N}$, $23^{\circ} 45^{\prime} 41^{\prime} \mathrm{E}, 690 \mathrm{~m}$, slope with various deciduous trees and herb and fern undergrowth, soil washing beneath old oak

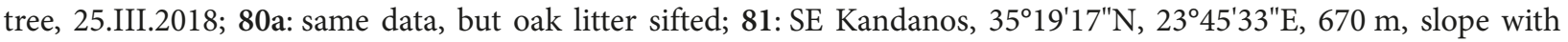
various deciduous trees and herb and fern undergrowth, soil washing near old oak tree, 25.III.2018; 82: Psiloritis, S Livadia, road to Mygerou refuge, $35^{\circ} 17^{\prime} 44^{\prime \prime} \mathrm{N}, 24^{\circ} 48^{\prime} 10^{\prime \prime} \mathrm{E}, 710 \mathrm{~m}$, relatively dry pasture with single old oak tree, soil washing, 26.III.2018; 83: Psiloritis, S Livadia, road to Mygerou refuge, $35^{\circ} 17^{\prime} 17^{\prime \prime} \mathrm{N}, 24^{\circ} 47^{\prime} 51^{\prime \prime} \mathrm{E}, 820 \mathrm{~m}$, slope with very old oak trees, shrubs, and grass, soil washing, 26.III.2018; 84: SE Malia, Selena Oros, 35²14'40"N, $25^{\circ} 31^{\prime} 03^{\prime \prime} \mathrm{E}, 880 \mathrm{~m}$, calcareous N-slope with Quercus ilex, soil washing, 26.III.2018; 85: NE Lassithi Plateau, 35²12'45"N, 2531'53"E, 850 m, dry ruderal stream valley with old Platanus, soil washing, 27.III.2018; 86: Dikti Oros, S Lassithi Plateau, Limnakaro, $35^{\circ} 08^{\prime} 09^{\prime \prime N}, 25^{\circ} 28^{\prime} 58^{\prime \prime E}, 1160$ m, Messor nest, 27.III.2018; 87: Dikti Oros, Limnakaro, 3508'40"N, $25^{\circ} 28^{\prime} 53^{\prime \prime E}, 1130$ m,

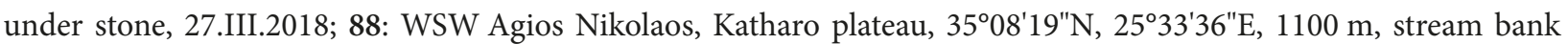

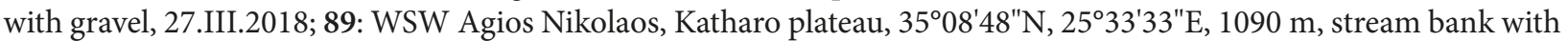

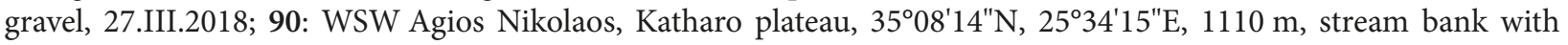
gravel, 28.III.2018.

December-January 2018/2019, leg. Assing: 91: SW Chania, WSW Lakki, 35²3'54"N, 2354'47"E, $430 \mathrm{~m}$, stream valley with Platanus and with Rubus and Ivy undergrowth, soil washing, 22.XII.2018; 92: SW Chania, WSW Lakki, 35²3'53"N,

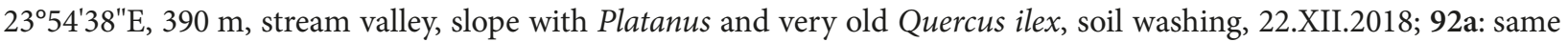
data, but near Platanus near stream; 92b: same data, but very old chestnut on slope near road; 93: SW Chania, Mavros stream valley, $35^{\circ} 23^{\prime} 48^{\prime \prime} \mathrm{N}, 23^{\circ} 53^{\prime} 40^{\prime \prime} \mathrm{E}, 410 \mathrm{~m}$, dark stream valley with old chestnut, deep moist layers of chestnut litter between stones sifted, 22.XII.2018; 94: S Kissamos, NW Elos, Limni, 35²2'16"N, 2337'55"E, 580 m, margin of artificial pond with old Platanus, soil washing, 23.XII.2018; 94a: same data, but debris and herb roots near pond sifted; 95: S Kissa-

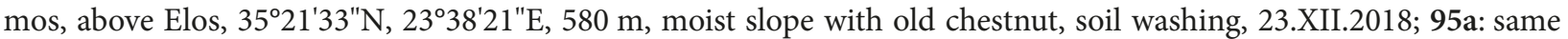

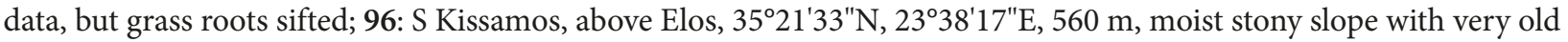
chestnut, very stony soil, soil washing, 23.XII.2018; 96a: same data, but bark of dead chestnut sifted; 97: NW Kandanos, Strovles, $35^{\circ} 22^{\prime} 03^{\prime \prime} \mathrm{N}, 23^{\circ} 40^{\prime} 07^{\prime \prime E}, 410 \mathrm{~m}$, stream valley with old chestnut and grass undergrowth, soil washing,

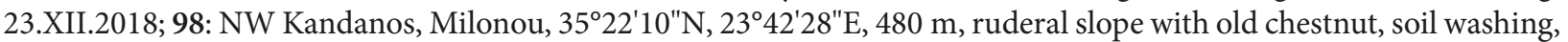
23.XII.2018; 99: SW Chania, S Nteres, $35^{\circ} 24^{\prime} 00^{\prime \prime} \mathrm{N}, 23^{\circ} 51^{\prime} 00^{\prime \prime} \mathrm{E}, 360 \mathrm{~m}$, stream valley with old chestnut and undergrowth

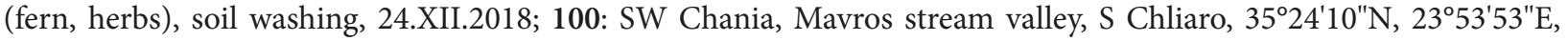
290 m, near stream bank, soil under old Platanus orientalis sifted, 25.XII.2018; 101: SW Chania, S Nteres, 35² $24^{\prime} 15^{\prime \prime} \mathrm{N}$, $23^{\circ} 50^{\prime} 58^{\prime \prime E}, 330 \mathrm{~m}$, slope with very old chestnut, fern, and herbs, soil washing, 25.XIII.2018; 102: ESE Rethimno,

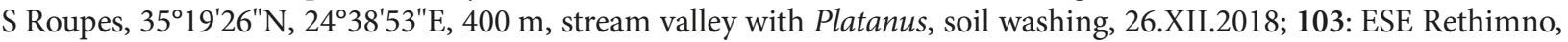

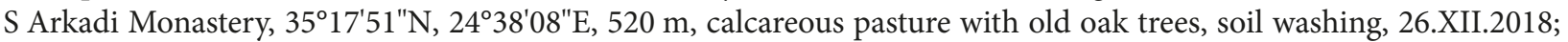

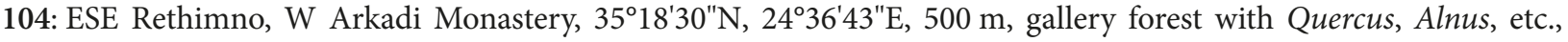
soil washing near stream, 26.XII.2018; 105: Psiloritis, NE Livadia, 35²18'56"N, $24^{\circ} 49^{\prime} 07^{\prime \prime} \mathrm{E}, 440 \mathrm{~m}$, stream valley with

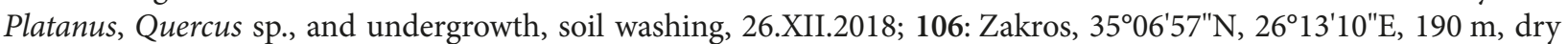

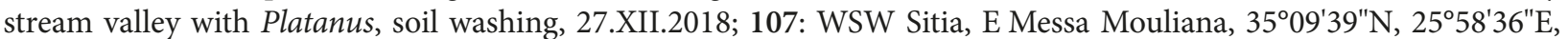

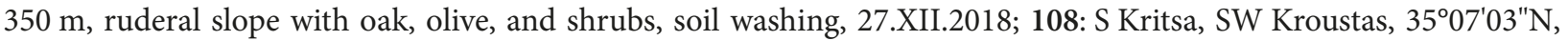
25 $38^{\prime} 10^{\prime \prime E}, 780 \mathrm{~m}$, forest with old Pinus and Quercus ilex, litter sifted, 28.XII.2018; 108a: same data, but pine bark sifted; 109: S Kritsa, SW Kroustas, $35^{\circ} 07^{\prime} 01^{\prime \prime N}, 25^{\circ} 37^{\prime} 55^{\prime \prime E}, 810 \mathrm{~m}$, forest margin with old Pinus and Quercus ilex, soil washing near old pine tree, 28.XII.2018; 109a: same data, but litter sifted, mostly near old pine tree; 109b: same data, but mush-

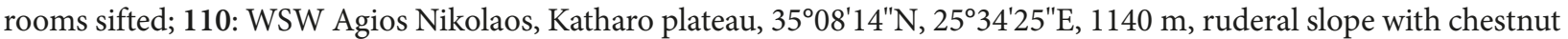
and Rubus undergrowth, litter and grass roots sifted and floated, 29.XII.2018; 111: WSW Agios Nikolaos, Katharo plateau, 350' $13^{\prime \prime} \mathrm{N}, 25^{\circ} 34^{\prime} 16^{\prime \prime E}, 1110 \mathrm{~m}$, moist ruderal slope, under stones, 29.XII.2018; 112: WSW Agios Nikolaos, Katharo plateau, $35^{\circ} 07^{\prime} 58^{\prime \prime} \mathrm{N}, 2^{\circ} 34^{\prime} 33^{\prime \prime} \mathrm{E}, 1160 \mathrm{~m}$, arable land, under stones, 29.XII.2018; 113: WSW Agios Nikolaos, Katharo plateau, $35^{\circ} 09^{\prime} 02^{\prime \prime} \mathrm{N}, 25^{\circ} 33^{\prime} 17^{\prime \prime} \mathrm{E}, 1090 \mathrm{~m}$, arable land, under stones, 29.XII.2018; 114: S Kritsa, SW Kroustas, $35^{\circ} 06^{\prime} 40^{\prime \prime} \mathrm{N}, 2^{\circ} 37^{\prime} 31^{\prime \prime E}, 960 \mathrm{~m}$, rocky slope with old Quercus ilex forest, litter sifted, 30.XII.2018; 115: NE Lassithi Plateau, $35^{\circ} 12^{\prime} 45^{\prime} \mathrm{N}, 25^{\circ} 31^{\prime} 53^{\prime} \mathrm{E}, 850 \mathrm{~m}$, dry ruderal stream valley with old Platanus, soil washing, 30.XIII.2018; 116: ENE Kastelli, $35^{\circ} 12^{\prime} 30^{\prime \prime} \mathrm{N}, 25^{\circ} 23^{\prime} 17^{\prime \prime} \mathrm{E}, 470 \mathrm{~m}$, slope with olive, oak trees, and ivy undergrowth, soil washing near oak trees, 30.XII.2018; 117: ENE Kastelli, 35 $12^{\prime} 35^{\prime \prime} \mathrm{N}, 25^{\circ} 22^{\prime} 12^{\prime \prime E}, 600 \mathrm{~m}$, oak trees between olive orchards, soil washing, 30.XII.2018; 118: E Kastelli, $35^{\circ} 12^{\prime} 27^{\prime \prime}$ N, $25^{\circ} 20^{\prime} 57^{\prime \prime} \mathrm{E}, 360 \mathrm{~m}$, stream valley with oak and Platanus, and with Rubus and herb undergrowth, soil washing, 30.XII.2018; 119: S Kritsa, SW Kroustas, 3507'03"N, 253' $18^{\prime \prime}$ E, 780 m, forest with old

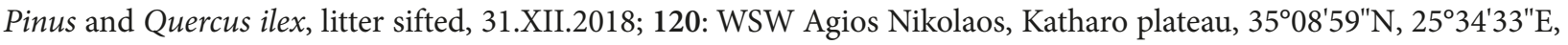
$1200 \mathrm{~m}$, margin of stony arable land and road margin, under stones, 1.I.2019.

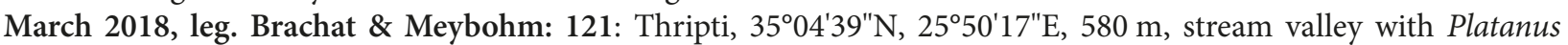
orientalis, litter sifted, 10.III.2018; 122: Thripti, $35^{\circ} 05^{\prime} 17^{\prime \prime} \mathrm{N}, 25^{\circ} 51^{\prime} 54^{\prime} \mathrm{E}, 880 \mathrm{~m}$, arable land, litter sifted, 10.III.2018; 123: Thripti, $35^{\circ} 05^{\prime} 09^{\prime \prime} \mathrm{N}, 25^{\circ} 52^{\prime} 10^{\prime \prime} \mathrm{E}, 1040 \mathrm{~m}$, litter sifted, 10.III.2018; 124: Lassithi, S Kritsa, 350' $55^{\prime \prime} \mathrm{N}, 25^{\circ} 38^{\prime} 18^{\prime \prime} \mathrm{E}$, 
$380 \mathrm{~m}$, litter in dry stream bed sifted, 11.III.2018; 125: Lassithi, S Kritsa, 350' $45^{\prime \prime} \mathrm{N}, 25^{\circ} 38^{\prime} 30^{\prime \prime} \mathrm{E}, 390 \mathrm{~m}$, stream valley

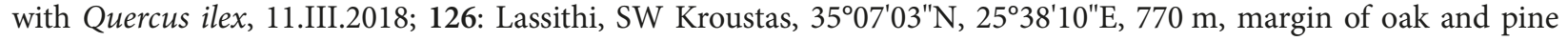
forest, litter sifted, 11.III.2018; 127: Lassithi, SW Kroustas, $35^{\circ} 07^{\prime} 03^{\prime \prime} \mathrm{N}, 25^{\circ} 38^{\prime} 10^{\prime \prime} \mathrm{E}, 770 \mathrm{~m}$, margin of oak and pine

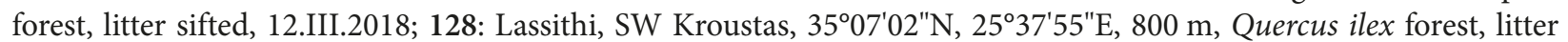
sifted, 12.III.2018; 129: Sfaka, 3508' $55^{\prime \prime} \mathrm{N}, 25^{\circ} 55^{\prime} 27^{\prime \prime} \mathrm{E}, 360 \mathrm{~m}$, litter sifted, 13.III.2018; 130: Kimouriotis, $35^{\circ} 10^{\prime} 40^{\prime \prime} \mathrm{N}$,

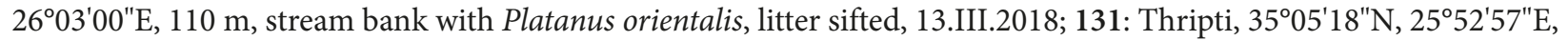

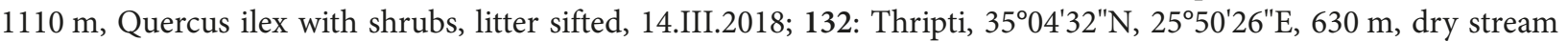
valley with Platanus orientalis, 14.III.2018; 133: Psiloritis, road from Anogia to Nida Plateau, $35^{\circ} 15^{\prime} 40^{\prime \prime} \mathrm{N}, 2^{\circ} 53^{\prime} 12^{\prime \prime} \mathrm{E}$, 1180 m, litter sifted, 15.III.2018; 134: Psiloritis, Nida Plateau, 35²12'18"N, 2450'04"E, 1380 m, litter sifted, 15.III.2018; 135: Lefka Ori, Omalos Plateau, $35^{\circ} 19^{\prime} 10^{\prime \prime} \mathrm{N}, 23^{\circ} 54^{\prime} 46^{\prime \prime E}, 1110 \mathrm{~m}$, litter sifted, 16.III.2018; 135a: same data, but 18.III.2018; 136: Lefka Ori, Omalos Plateau, 35²19'23"N, 2351'59"E, 1100 m, litter sifted, 17.III.2018; 137: Lefka Ori, Omalos Plateau, $35^{\circ} 19^{\prime} 28^{\prime \prime} \mathrm{N}, 23^{\circ} 53^{\prime} 06^{\prime \prime E}, 1060$ m, litter sifted, 17.III.2018; 138: Lefka Ori, Omalos Plateau, 35¹9'08"N, $23^{\circ} 55^{\prime} 10^{\prime \prime} \mathrm{E}, 1200 \mathrm{~m}$, valley with Acer sp., litter sifted, 17.III.2018; 139: Lefka Ori, Omalos Plateau, 35²0'57"N,

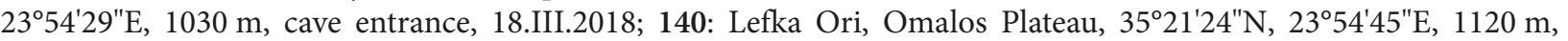

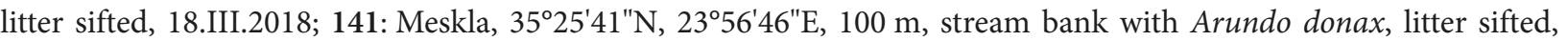

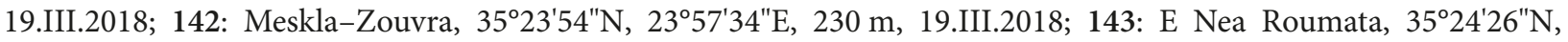

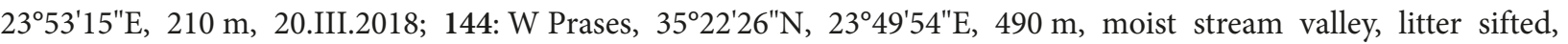

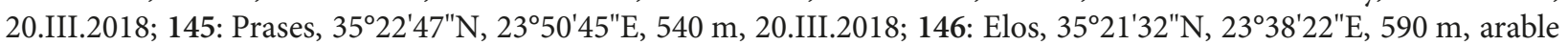
land with old chestnut trees, litter sifted, 21.III.2018; 147: Elos, 35 $21^{\prime} 21^{\prime \prime} \mathrm{N}, 23^{\circ} 38^{\prime} 21^{\prime \prime} \mathrm{E}, 610 \mathrm{~m}$, arable land with old chestnut trees, litter sifted, 21.III.2018; 148: Strovles, $35^{\circ} 22^{\prime} 17^{\prime \prime} \mathrm{N}, 23^{\circ} 40^{\prime} 03^{\prime \prime} \mathrm{E}, 380 \mathrm{~m}$, litter of Platanus orientalis sifted, 21.III.2018; 149: $10 \mathrm{~km}$ W Omalos, Prases, 3521'31"N, 2349'29"E, 760 m, N-slope with Erica arborea, Arbutus sp., and Platanus orientalis, litter sifted, partly near rotting tree trunk, 22.III.2018; 150: Nteres, $35^{\circ} 24^{\prime} 50^{\prime \prime} \mathrm{N}, 23^{\circ} 50^{\prime} 47^{\prime \prime} \mathrm{E}$, $285 \mathrm{~m}$, dry stream valleys with chestnut trees, litter sifted, 23.III.2018; 151: Nteres, $35^{\circ} 25^{\prime} 07^{\prime \prime} \mathrm{N}, 23^{\circ} 50^{\prime} 54^{\prime \prime} \mathrm{E}, 210 \mathrm{~m}$, litter of Platanus orientalis sifted, 23.III.2018; 152: Nteres, $35^{\circ} 25^{\prime} 48^{\prime \prime} \mathrm{N}, 23^{\circ} 50^{\prime} 37^{\prime \prime} \mathrm{E}, 200 \mathrm{~m}$, litter of Platanus orientalis sifted,

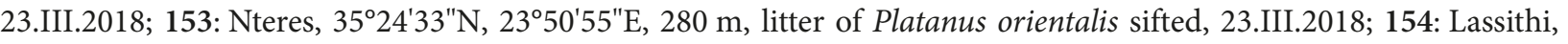

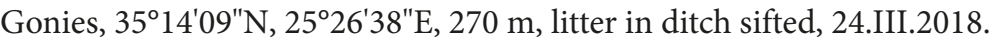

March 2019, leg. Brachat \& Meybohm: 155: Ano Zakros, 3506'57"N, 26²13'10"E, $190 \mathrm{~m}$, stream valley, leaf litter sifted

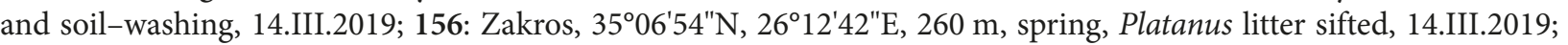
157: Kimouriotis, $35^{\circ} 10^{\prime} 40^{\prime \prime} \mathrm{N}, 26^{\circ} 03^{\prime} 00^{\prime \prime} \mathrm{E}, 110 \mathrm{~m}$, stream valley with Platanus orientalis, litter sifted and soil washing,

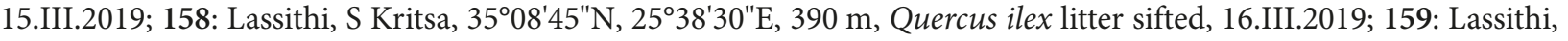
SW Kroustas, $35^{\circ} 07^{\prime} 02^{\prime \prime} \mathrm{N}, 25^{\circ} 37^{\prime} 55^{\prime} \mathrm{E}, 800 \mathrm{~m}$, Quercus ilex litter sifted, soil washing, and under stones, 16.III.2019; 160: Psiloritis, NE Sisarcha, $35^{\circ} 18^{\prime} 04^{\prime \prime} \mathrm{N}, 24^{\circ} 54^{\prime} 47^{\prime \prime} \mathrm{E}, 570 \mathrm{~m}$, stream bank with Platanus orientalis, litter sifted and soil washing, 17.III.2019; 161: Psiloritis, Anogia-Psiloritis, 35²15'22"N, 2453'04"E, 1200 m, Quercus ilex, Acer, and Berb-

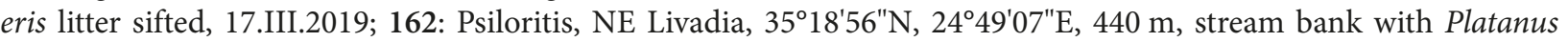
orientalis, litter sifted and soil washing, 18.III.2019; 163: Psiloritis, S Livadia, 35 ${ }^{\circ} 17^{\prime} 19^{\prime \prime} \mathrm{N}, 24^{\circ} 47^{\prime} 52^{\prime \prime} \mathrm{E}, 810 \mathrm{~m}$, litter of

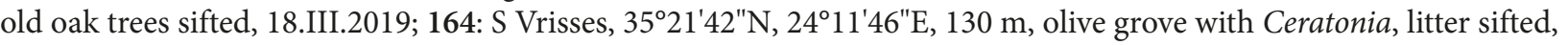

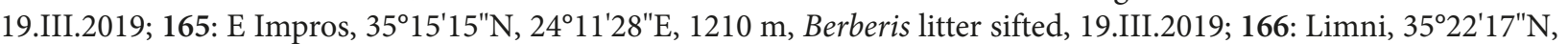
$23^{\circ} 37^{\prime} 57^{\prime \prime E}, 590 \mathrm{~m}$, margin of artificial pond, sifted, 20.III.2019; 167: Louchi, 35²1'56"N, 23³7'16"E, $550 \mathrm{~m}$, arable land with chestnut and olives, litter and bark sifted, 20.III.2019; 168: W Karanos, 35²3'54"N, 235ㄴ'38"E, 440 m, stream valley, Platanus orientalis litter sifted and soil washing, 21.III.2019; 169: S Vrisses, 35² $21^{\prime} 42^{\prime \prime} \mathrm{N}, 24^{\circ} 11^{\prime} 46^{\prime \prime} \mathrm{E}, 130 \mathrm{~m}$,

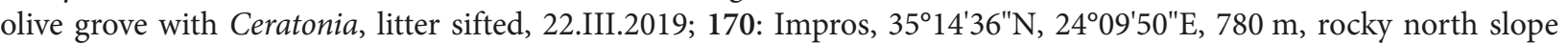

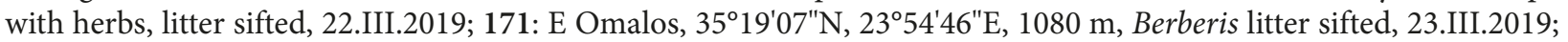

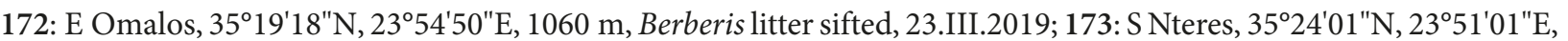
$340 \mathrm{~m}$, stream bank with Platanus orientalis, litter sifted and soil washing, 24.III.2019; 174: E Omalos, 35²19'07"N, 2354'46"E, 1080 m, Berberis litter sifted, 25.III.2019; 175: S Omalos, 35¹9'22"N, 2353'33"E, 1050 m, Berberis litter

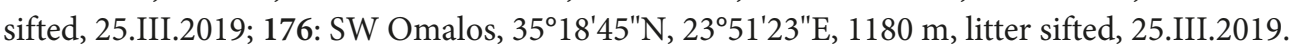

March 2001, leg. Meybohm: 177: Orno Thriptis, $35^{\circ} 05^{\prime} \mathrm{N}, 25^{\circ} 52^{\prime} \mathrm{E}, 900 \mathrm{~m}$, 8.III.2001; 178: Orno Thriptis, $35^{\circ} 05^{\prime} \mathrm{N}$, $25^{\circ} 52^{\prime} \mathrm{E}, 1000 \mathrm{~m}$, 8.III.2001; 179: Orno Thriptis, $35^{\circ} 05^{\prime} \mathrm{N}, 25^{\circ} 52^{\prime} \mathrm{E}, 1000 \mathrm{~m}$, 11.III.2001; 180: Dikti Oros, Sela-

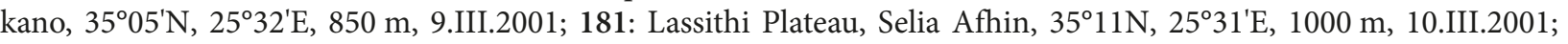

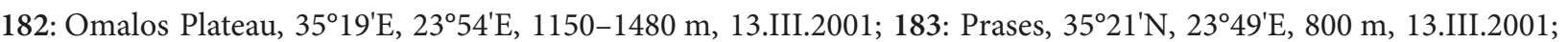

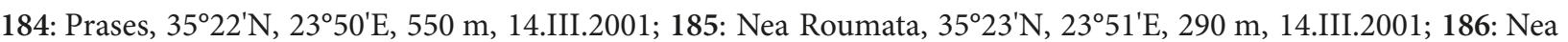

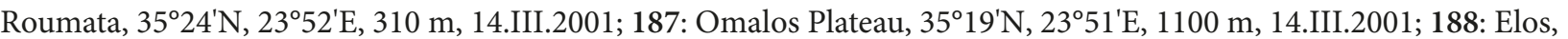

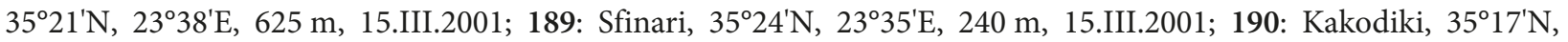

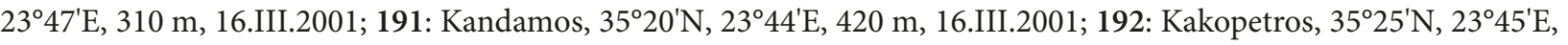
500 m, 16.III.2001; 193: Rethimnon, Armeni, $35^{\circ} 17^{\prime} \mathrm{N}, 24^{\circ} 28^{\prime} \mathrm{E}$, oak forest, 17.III.2001.

October 1991, leg. Wunderle: 194: SW Chania, Nteres env., 400 m, stream bank, sifted, 5.X.1991; 195: same data, 13.X.1991; 196: same data, but floated, 10.X.1991; 197: Nteres env., 500 m, stream bank, floated and sifted from moss and debris, 13.X.1991; 198: E Chania, estuary, debris on sandy beach sifted, 6.X.199; 199: W Chania, beach, debris 
and seaweed on sand sifted, 7.X.1991; 200: Vathi env., $350 \mathrm{~m}$, spring with moist leaf litter, sifted, 7.X.1991; 201: Elos env., 300 m, chestnut litter sifted, 7.X.1991; 202: Omalos plateau, 1200 m, plant debris between rocks sifted, 8.X.1991; 203: Omalos plateau, 1200 m, debris between rocks sifted, 13.X.1991; 204: Psilorithis, Axos env., 500 m, macchia, litter sifted, 10.X.1991; 205: Nida plateau, 1550 m, phrygana, litter sifted, 10.X.1991; 206: SW Chania, Prases, 700 m, pine litter sifted, 13.X.1991; 207: Rethimnon, Koxare env., 500 m, Quercus ilex forest, 16.X.1991; 208: Preveli, coast, stream bank floated and tree fungi sifted, 16.X.1991.

2016-2018, leg. Giachino \& Vailati, subterranean pitfall traps: 209: Chania, road Seli Koulouridiana, $35^{\circ} 20^{\prime} 52^{\prime \prime} \mathrm{N}$, 234' 14"E, 730 m, VI.2016-VI.2018; 210: Chania, Kares, Agios Paulos valley, 35²1'52"N, 2404'30"E, 960 m, VI.2016VI.2018; 211: Chania, Amoudhari, Tavri Refuge env., 35¹7'32"N, 2409'25"E, 1250 m,VI.2016-VI.2018; 212: Chania,

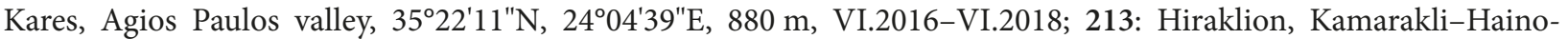

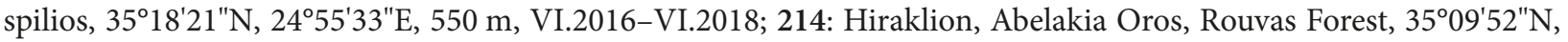
245ㄴ'36"E, 1030 m, VI.2016-VI.2018; 215: Hiraklion, Abelakia Oros, Diplori, 3509'30"N, 2456'11"E, 1320 m, VI.2016-VI.2018; 216: Rethimno, Kourouna near Amigdalo, 35²14'48"N, 2448'56"E, 1190 m, VI.2016-VI.2018;

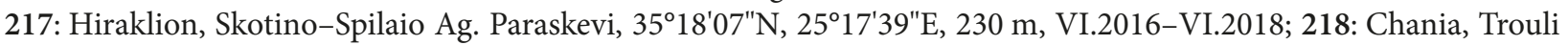
Oros, 35¹8'49"N, 2351'19"E, 990 m, VI.2016-VI.2018.

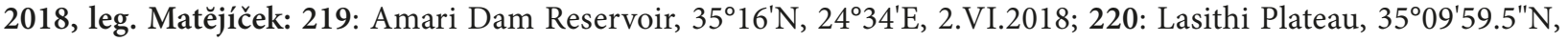
2531'27.9"E, 25.VIII.-5.IX.2018; 221: Avrakontes env., 3509'N, 2529'E, 25.VIII.-5.IX.2018; 222: Adelianos Kampos, $35^{\circ} 22^{\prime} \mathrm{N}, 24^{\circ} 33^{\prime} \mathrm{E}$, at light, 30.V.-6.VI.2018; 223: Kourtalioti Canyon, $35^{\circ} 12^{\prime} \mathrm{N}, 24^{\circ} 28^{\prime} \mathrm{E}$, on sand near stream, 30.V.2018.

October 2009, leg. Fouqué: 224: $35^{\circ} 18^{\prime} \mathrm{N}, 24^{\circ} 30^{\prime} \mathrm{E}, 520 \mathrm{~m}$, 9.X.2009; 225: Omalos plateau, $35^{\circ} 20^{\prime} \mathrm{N}, 23^{\circ} 54^{\prime} \mathrm{E}, 1050 \mathrm{~m}$, 5.X.2009; 226: Nida plateau, 35²12'N, 24'50'E, 1350 m, 11.X.2009; 227: Nida to Psiloritis, 1500-2000 m, 8.X.2009.

1979-2000, various collectors: 228: Zakros, 19.IX.1979, leg. Fülscher; 229: Zakros, 29.V.1980, leg. Brachat; 230: Ano Zakros, 20.III.1986, leg. Meybohm; 231: Kato Horio, 200 m, 14.IV.2000, leg. Meybohm; 232: Itanos, 19.III.1986,

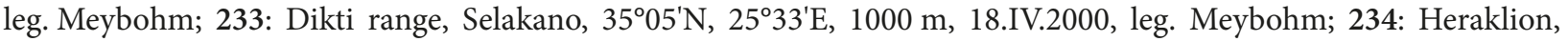
NW Kasteliana, 3.IV.1980, leg. Brachat; 235: Heraklion, Pefkos env., 5.IV.1983, leg. Brachat \& Dörnfeld; 236: Rethimno, Garazo env., 25.V.1980, leg. Brachat; 237: Rethimno, Anogia, 25.V.1980, leg. Brachat; 238: Rethimno, Anogia - Ideon Antron, 25-27.V.1980, leg. Brachat; 239: Rethimno, Petres river, gravel bank, 29.V.1981, leg. Mühle; 240: Chania, Gulf of Georgiopoulioli, 23-24.V.1980, leg. Brachat; 241: Chania, Vrises, 19.V.1980, leg. Brachat; 242: Chania, Vrises env., 19.III.1983, leg. Brachat \& Dörnfeld; 243: Chania, Kalives env., Kiliaris river, 23.V.1980, leg. Brachat; 244: Rethimno, Spili env., Dariviana, 3.IV.1982, leg. Brachat \& Dörnfeld; 245: Chania, Omalos plateau, 21-22.V.1980, leg. Brachat; 246: Chania, Omalos plateau, 24.III.1986, leg. Meybohm; 247: Chania, Omalos-Samaria, 20.V.1980, leg. Brachat; 248: Chania, Samaria Gorge, 28.III.1983, leg. Brachat \& Dörnfeld; 249: Chania, Samaria Gorge, 2-4.VI.1981, leg. Mühle; 250: Chania, Sfinari-Kampos, 27.III.1983, leg. Brachat \& Dörnfeld; 251: Chania, Skaloti env., 31.III.1983, leg. Brachat \& Dörnfeld; 252: Lassithi, Lithines env., 30.V.1980, leg. Brachat; 253: Chania, Elos, env., 25.III.1986, leg. Meybohm; 254: Chania, Lakk-Phrunes, IV.1986, leg. Franz; 255: Heraklion, Vôri (Phaistos), 26.VII.1979, leg. Senglet (MHNG); 256: West Crete, Kournas lake, lake shore, 10.VI.2002, leg. Feldmann (cFel); 257: West Crete, Georgioupoli, beach, IV.1997, leg. Feldmann (cFel); 257a: same data, but VI.2002 (cFel).

\section{References:}

A95 = Assing (1995); A97a = Assing (1997a); A97b = Assing (1997b); A99 = Assing (1999); A00 = Assing (2000); A01 = Assing (2001); A02 = Assing (2002); A03a = Assing (2003a); A03b = Assing (2003b); A03c = Assing (2003c); A04a = Assing (2004a); A04b = Assing (2004b); A05a = Assing (2005a); A05b = Assing (2005b); A06 = Assing (2006); A07a = Assing (2007a); A07b = Assing (2007b); A07c = Assing (2007c); A07d = Assing (2007d); A07e = Assing (2007e); A07f = Assing (2007f); A08a = Assing (2008a); A08b = Assing (2008b); A08c = Assing (2008c); A08d = Assing (2008d); A08e = Assing (2008e); A08f = Assing (2008f); A09a = Assing (2009a); A09b = Assing (2009b); A09c = Assing (2009c); A09d = Assing (2009d); A10a = Assing (2010a); A10b = Assing (2010b); A10c = Assing (2010c); A12 = Assing (2012); A13a = Assing (2013a); A13b = Assing (2013b); A13c = Assing (2013c); A14a = Assing (2014a); A14b = Assing (2014b); A15a = Assing (2015a); A15b = Assing (2015b); A17a = Assing (2017a); A17b = Assing (2017b); A18a = Assing (2018a); A18b = Assing (2018b); A18c = Assing (2018c); A18d = Assing (2018d); A19a = Assing (2019a); App = Assing (present paper); AV19 = Assing \& Vogel (2019); AW95 = Assing \& Wunderle (1995); AW08 = Assing \& Wunderle (2008); BB16 = Blattný \& Blattný (1916); Be55 = Besuchet (1955); Bn44 = Brundin (1944); Bo75 = Bordoni (1975); Bo76 = Bordoni (1976); Bo77 = BordonI (1977); Bo84 = Bordoni (1984); Bo86 = Bordoni (1986); Bo07 = Bordoni (2007); Bo09 = Bordoni (2009); Bo14 = Bordoni (2014); Bo16 = Bordoni (2016); Bol7 = Bordoni (2017); Br15 = BERnHAuer (1915); Br35 = BERNHAUER (1935); Br39 = Bernhauer (1939); C70 = CoIfFAIt (1970); C72 = CoIfFAIT (1972); C73 = CoIfFait (1973); C76 = Coiffait (1976); C80 = Coiffait (1980); Ca96 = CAstellini (1996); Cu17 = CuCCodoro (2017); F51 = FAGEL (1951); Fr94 = FrISCH (1994); Fr97 = FrISCH (1997); Fr98 = FrISCH (1998); Fr99a = FrISCH (1999a); Fr99b = FrISCH (1999b); Fr10 = Frisch (2010); Fz71 = Franz (1971); G15 = Gildenkov (2015); He84 = Heyden (1884); Ho63 = 
Horion (1963); Ho65 = Horion (1965); Ho67 = Horion (1967); JH06 = JASZAY \& HLAváČ (2006); Ka55 = KARAMAN (1955); Ko36 = Koсн (1936); Kz57 = KraAtz (1857); Kz58a = KraAtz (1858a); Kz58b = KraAtz (1858b); Lö98 = LÖBL (1998); LS = LOHSE \& STEEL (1961); Ly13 = LoKAY (1913); Ly21 = LOKAY (1921); M08 = MeYBOHM (2008); Ma14 = Makranczy (2014); Mn35 = MaŘan (1935); O87 = Oertzen (1887); P68 = Puthz (1968); P71 = Puthz (1971); P72a = Puthz (1972a); P72b = Puthz (1972b); P77 = Puthz (1977); P79 = Puthz (1979); P80 = Puthz (1980); P08 = Puthz (2008); Pa96 = Pace (1996); Pa02 = PACe (2002); Pi03 = PiC (1903); R85 = Reitter (1885); Sa03 = SAHLberg (1903); Sb02 = SAbella (2002); SBB98 = SABELla et al. (1998); SBBB04 = SABELla et al. (2004); Sc09 = SCHÜLKE (2009); Sc10 = SCHÜLKe (2010); Sc12 = SCHÜLKe (2012); Sc19 = SCHÜLKe (2019); SK00 = SchülKe \& KociAN (2000); SS15 = SCHÜlKe \& SMETANA (2015); Sz36 = SCheERPELtZ (1936); Sz64 = SCHEERPELtZ (1964); Z88 = ZerCHE (1988); Z90 = ZERCHE (1990); Z91 = ZERCHE (1991).

Footnotes: ${ }^{1)}$ Reported as Mycetoporus sp. aff. bosnicus Luze, 1901. ${ }^{2)}$ Reported as Myrmecopora sp. ${ }^{3)}$ Reported as Ocalea badia ERICHSON, 1837. ${ }^{4)}$ Reported as Oxypoda (brachyptera group) sp. ${ }^{5}$ Reported as Quedius umbrinus ERICHSON, 1839. ${ }^{6}$ Reported as Ocypus picipennis (FABricius, 1792). ${ }^{7)}$ Reported as Brachygluta sp. n. ${ }^{8)}$ Cited as Tychus sp. n. ${ }^{9)}$ Reported as Atheta sp. ${ }^{10)}$ Reported as Stenus cf. cordatoides Puthz, 1972. ${ }^{11)}$ Reported as Quedius candicus CoIffaIT, 1976 (synonym). ${ }^{12)}$ Reported as Dolicaon venustus BAUdI, 1848 (misidentification; see A05). ${ }^{13)}$ Reported as Xantholinus relucens (GravenHorst, 1806) (misinterpretation). ${ }^{14)}$ Reported as Xantholinus piochardi CoIfFaIt, 1956, synonym of $X$. rufipennis ErICHson, 1839. ${ }^{15)}$ Reported as X. graecus KraAtz, 1858. ${ }^{16)}$ Reported as Neobisnius cerrutii Gridelli, 1943 (synonym). ${ }^{17)}$ Reported as Philonthus bimaculatus (GravenHorst, 1802) (synonym). ${ }^{18)}$ The record of Quedius pallipes Lucas, 1846 probably refers to Q. levicollis (misidentification). ${ }^{19)}$ Reported as Quedius rufipes (GravENHORST, 1802) (synonym). ${ }^{20)}$ Reported as Xantholinus rufipennis ERICHson, 1839. ${ }^{21)}$ Reported as Lobrathium apicale (BAUDI Di SElve, 1857). ${ }^{22)}$ Reported as Pronomaea rostrata (ERICHSON, 1837). ${ }^{23)}$ Reported as Falagria sulcata (PAYKULL, 1780). ${ }^{24)}$ Reported as Atheta (Coprothassa) sordida (MARsham, 1802) or Homalota sordida, respectively. ${ }^{25)}$ Reported as Oxypoda sericea HeEr, 1839 (synonym). ${ }^{26)}$ Reported as Geostiba minoica PACE, 1996 (synonym). ${ }^{27)}$ Reported as Quedius tristis (Gravenhorst). ${ }^{28)}$ Reported as Quedius candicus CoIffait, 1976 (synonym). ${ }^{29)}$ The record of G. ripicola (KIESENWETTER, 1844) most likely refers to G. rubrior. ${ }^{30)}$ Reported as Leptobium minos BordonI, 1984 (synonym). ${ }^{31)}$ Reported as Domene losheianum [sic] Bordoni, 1977 (synonym). ${ }^{32)}$ Record of Xantholinus rufipennis ERICHSON, 1839 probably based on a misidentification and referring to this species. ${ }^{33)}$ Reported as Stenus cres PUTHZ, 1971 (synonym). ${ }^{34)}$ The record of Atheta elongatula (GRAVEnHORst, 1802) probably refers to this species. ${ }^{35)}$ The record of Habrocerus capillaricornis (GravenHorst, 1806) most likely refers to this species. ${ }^{36}$ ) Reported as Tachyporus discus ReICHE \& SAULCY, 1856. ${ }^{37)}$ The record of Neobisnius procerulus (GravenHORST, 1802) most likely refers to this species. ${ }^{38)}$ Reported as Leptobium melanocephalum (Reiche \& SAUlcy, 1856). ${ }^{39)}$ Reported as Achenium levantinum ReITTER, 1884 (synonym). ${ }^{40)}$ The record of Medon fusculus (MANnerheim, 1830) most likely refers to this species. ${ }^{41)}$ The record of Sunius melanocephalus (FABricius, 1792)) most likely refers to this species. ${ }^{42)}$ The record of Astenus angustatus (PAYKull, 1789) most likely refers to this species. ${ }^{43)}$ The record of Platystethus cornutus "GylL." most likely refers to this species. ${ }^{44)}$ The record of Anotylus speculifrons (KRAATz, 1857) most likely refers to this species. ${ }^{45)}$ The record of Bledius tricornis (HERBST, 1784) most likely refers to this species. ${ }^{46)}$ Reported as Liogluta vicina (STEPHENs, 1832) (synonym). ${ }^{47)}$ Reported as Atheta pertyi (HeER, 1839) (misinterpreted name). ${ }^{48)}$ The female-based record of Ochthephilum collare (REITTER 1884) probably refers to this species. ${ }^{49)}$ Reported as Gyrohypnus punctulatus (PAYKULL, 1789) (misinterpretation). ${ }^{50)}$ Reported as Xantholinus graecus calcidicus Bordoni, 1973. ${ }^{51)}$ Reported as Philonthus agilis (GravenHorst, 1806) (synonym). ${ }^{52)}$ Reported as Stenus unicolor ERICHSON, 1840 (synonym). ${ }^{53)}$ Reported as Stenus rusticus ERICHSON, 1840 (synonym). ${ }^{54)}$ Reported as Tachyporus cerrutii CoIffait, 1980 (synonym). ${ }^{55)}$ Reported as Tachyporus brunneus (FABricius, 1793) (synonym). ${ }^{56)}$ Reported as Paederus longipennis ERICHson, 1839 (synonym). ${ }^{57)}$ Reported as Lesteva maura Erichson, 1840. ${ }^{58)}$ Reported as Phytosus holtzi Bernhauer, 1935 (synonym). ${ }^{59)}$ Reported as Tyrus mucronatus (PAnzer, 1803). ${ }^{60)}$ Reported as Bryaxis sanguinea. ${ }^{61)}$ The record of Bibloplectus ambiguus (ReICHEnBACH, 1816) probably refers to this species. ${ }^{62)}$ The record of Pselaphus heisei Herbst, 1791 probably refers to this species. ${ }^{63)}$ Reported as Euplectus revelierei ReITTER, 1884 (synonym). ${ }^{64)}$ Reported as Brachygluta schuppelii (AuBÉ, 1844) (synonym). ${ }^{65)}$ Listed only for Rhodos in SchüLke \& SMETANA (2015), but recorded from Crete by Assing (2013a). ${ }^{66)}$ Reported as Eutheia schaumii Kiesenwetter, 1858 (misidentification). ${ }^{67)}$ Reported as Hydrosmecta sp. ${ }^{68)}$ Reported as Phloeocharis subtilissima MANnerheIm, 1830 (misidentification). ${ }^{69)}$ Reported as Phloeocharis hummleri BernHAUER, 1915 (synonym); ${ }^{70)}$ Reported as Atheta biroi SCHEERPELTz, 1964 (synonym). ${ }^{71)}$ Reported as Leptomastax bisetosa REITTER, 1884. ${ }^{072)}$ Reported as Heterothops minutus Wollaston, 1860 (misidentification). ${ }^{73)}$ Reported as Typhlocyptus pandellei SAulcy, 1878. ${ }^{74)}$ Reported as Amischa sp. ${ }^{75)}$ Reported as Oxypoda (Baeoglena) sp. ${ }^{76)}$ Reported as Gynotyphlus sp. n. 77) Reported as Faronus lafertei Aubé, 1844 (misidentification) (BRACHAT pers. comm.). ${ }^{78)}$ Previous records of Stenichnus aegialius (ReITTER, 1884) most likely refer to this species. ${ }^{79)}$ Reported as Pseudobium hellenicum Assing, 2006. ${ }^{80)}$ The record of Sepedophilus nigripennis (STEPHENS, 1832) most likely refers to this species. ${ }^{81)}$ Reported as Oxypoda formosa KraAtz, 1856. ${ }^{82)}$ Reported as Mycetoporus dispersus SchülKe \& Kocian, 2000 (synonym). ${ }^{83)}$ Reported as Pseudolathra cretensis Bordoni, 1986 (synonym). 


\subsection{Unidentified and unnamed species}

A reliable identification at the species level was not possible for some of the recently collected material because it was represented by females only or because the respective taxon is currently in a state of taxonomic confusion (Hydrosmecta Thomson, 1858, Microdota Mulsant \& Rey, 1873, Mocyta Mulsant \& Rey, 1874). Some species are undoubtedly undescribed, but remain unnamed for want of males.

Tab. 2: Unidentified and unnamed species.

\begin{tabular}{|c|c|c|}
\hline Species & Localities/Samples & References \\
\hline \multicolumn{3}{|l|}{ Pselaphina e } \\
\hline${ }^{*}$ Afropselaphus $\geq 2$ spp. ( & $92(1), 125(2), 138(1), 158(1), 175(1)$ & A13a, A15a, App \\
\hline Amauronyx sp. ( \&) & $83(1), 84(1)$ & \\
\hline Bryaxis sp. (female) & $85(1)$ & \\
\hline${ }^{\star}$ Euplectus sp. n. 1 ( ( ) & $66(1), 105(1)$ & \\
\hline${ }^{\star}$ Euplectus sp. n. 2 ( ㅇ ) & $92 b(1)$ & \\
\hline${ }^{\star}$ Faronus sp. ( ㅇ ) & $136 \mathrm{a}(1), 137(1)$ & \\
\hline \multicolumn{3}{|l|}{ Aleocharinae } \\
\hline Atheta (Ceritaxa) sp. ( & $15(1), 212(1)$ & \\
\hline Atheta (Microdota) sp. & $136(1)$ & A13a \\
\hline${ }^{* *}$ Cousya sp. n. ( $(+)$ & & App \\
\hline Cypha sp. (ㅇ) & $187(1)$ & \\
\hline Hydrosmecta sp. 1 & $88(1), 90(2)$ & A13a \\
\hline Hydrosmecta sp. 2 & $89(1), 90(8)$ & A15a \\
\hline Hydrosmecta sp. 3 & & A15a \\
\hline${ }^{\star}$ Leptusa sp. n. ( + ) & $41 \mathrm{a}(1), 84(1)$ & \\
\hline Mocyta $\geq 2$ spp. & $\begin{array}{l}\text { 1(1), 8(1), 9(1), 18a(5), 19(1), 23(1), 39(1), 41(1), 41a(5), 42(4), 48(1), } \\
50(3), 53 \mathrm{a}(1), 56(1), 68(21), 62(2), 67(2), 74(1), 75(2), 82(4), 93(1), \\
95(1), 95 \mathrm{a}(50), 97(5), 103(2), 115(1), 121(10), 123(1), 124(3), 126(1), \\
128(1), 129(13), 130(1), 131(1), 135(1), 139(1), 141(1), 142(10), \\
143(4), j 144(2), 147(5), 149(3), 150(11), 153(11), 154(8), 157(1), \\
159(1), 160(2), 161(1), 162(1), 164(7), 165(7), 166(21), 167(10), 168(8), \\
169(6), 170(15), 171(1), 172(2), 175(1), 219(2), 220(2), 222(6), 223(2)\end{array}$ & A13a, A15a \\
\hline Myllaena sp. & & App \\
\hline Oxypoda (Thliboptera) sp. ( + ) & $170(1)$ & \\
\hline \multicolumn{3}{|l|}{ Scydma eninae } \\
\hline${ }^{* * C e p h e n n i u m ~ s p p . ~(~} 9$ ) & $2(1), 128(1)$ & ABM19 \\
\hline${ }^{* *}$ Leptomastax sp. n. ( ( ) & 159(1) & \\
\hline
\end{tabular}

\subsection{Records of doubtful status}

Species reported from Crete in older articles are considered doubtful or erroneous under the following conditions:

a) a revision of reference material proved the record to be erroneous;

b) records of species with restricted distributions whose presence in Crete would be unlikely;

c) records of species of taxonomically difficult groups that can be identified only based on an examination of the genitalia (e.g., Aloconota spp., Atheta spp.), which was not common practice at the time of the publication of a record; d) records of species belonging to groups in which additional similar species were described after the publication of a record;

e) records of species of taxonomically difficult groups (especially Aleocharinae) whose reliable identification would have required the expertise of a specialist of the respective taxon;

f) records of species based on misinterpreted (type) localities. 
The following records with names of doubtful identity were omitted from both Tab. 1 and Tab. 3: "Megarthrus affinis MiLL.", "Mycetoporus nanus ER.", "Mycetoporus splendens MARsh., "Phloeopora reptans GRAV.", "Scopaeus microphthalmus Fauv. i. 1., "Philonthus agilis Grav.", "Quedius ochripennis v. variabilis ReY" (all OeRTzen 1887), "Mycetoporus nanus ER.", “Oxytelus depressus Grav." (both SAhlberg 1903), Ocypus micropterus Brullé (KraAtz 1858a), "Oxytelus depressus Grav." (KRAATZ 1858b).

Tab. 3: Species erroneously or doubtfully recorded from Crete.

\begin{tabular}{|c|c|}
\hline Species & References \\
\hline \multicolumn{2}{|l|}{ Omalina e } \\
\hline $\begin{array}{l}\text { Omalium allardii FAIRMAIRE \& BRISOUT, } \\
1859\end{array}$ & Ho63, O87 \\
\hline \multicolumn{2}{|l|}{ Pselaphinae } \\
\hline Articerodes syriacus (SAULCY, 1865) & $\mathrm{Pi} 03$ \\
\hline $\begin{array}{l}\text { Bibloplectus ambiguus (REICHENBACH, } \\
\text { 1816) }\end{array}$ & BB16 \\
\hline $\begin{array}{l}\text { Brachygluta lefebvrei lefebvrei (AuBÉ, } \\
1833 \text { ) }\end{array}$ & Kz58a, O87 \\
\hline Bryaxis puncticollis (DENNY, 1825) & BB16 \\
\hline Trimium zoufali Krauss, 1900 & $\mathrm{BB} 16$ \\
\hline \multicolumn{2}{|l|}{ Tachyporinae } \\
\hline $\begin{array}{l}\text { Mycetoporus angularis Mulsant \& REY, } \\
1853\end{array}$ & Ho67, C76 \\
\hline $\begin{array}{l}\text { Sepedophilus pedicularius (GRAVEN- } \\
\text { HORST, 1802) }\end{array}$ & O87 \\
\hline \multicolumn{2}{|l|}{ Aleocharinae } \\
\hline Aleochara crassicornis LACORDAIRE, 1835 & Sz64 \\
\hline Aloconota cambrica (Wollaston, 1855) & $\mathrm{Sa} 03$ \\
\hline Aloconota insecta (Thomson, 1856) & O87 \\
\hline Aloconota sulcifrons (STEPHENS, 1832) & C76 \\
\hline Atheta basicornis (Mulsant \& ReY, 1852) & $\mathrm{Sa} 03$ \\
\hline Atheta corvina (Тномson, 1856) & O87 \\
\hline Atheta crassicornis (FABRICIUs, 1792) & C76 \\
\hline Atheta mortuorum (Тномson, 1867) & $\mathrm{Sa} 03$ \\
\hline $\begin{array}{l}\text { Atheta (Mocyta) clientula (ERICHSON, } \\
\text { 1839) }\end{array}$ & $\begin{array}{l}\text { C76, Kz58a, } \\
\text { Sz64 }\end{array}$ \\
\hline $\begin{array}{l}\text { Atheta (Mocyta) fungi (GRAVENHORsT, } \\
\text { 1806) }\end{array}$ & He84, Sz64 \\
\hline Atheta (Mocyta) orbata (ERICHSON, 1837) & Sz64 \\
\hline Atheta (Mocyta) pulchra (KrAATz, 1856) & O87 \\
\hline $\begin{array}{l}\text { Atheta (Mocyta) rhodiensis SCHEER- } \\
\text { PLETZ, } 1963\end{array}$ & Sz64 \\
\hline $\begin{array}{l}\text { Cypha apicalis (BRISOUT DE BARNEVILLE, } \\
\text { 1863) }\end{array}$ & Sz64 \\
\hline
\end{tabular}

\begin{tabular}{|c|c|}
\hline Species & References \\
\hline Myllaena graeca KRAATZ, 1858 & $\begin{array}{l}\text { A18d, } \\
\text { Kz58a, O87 }\end{array}$ \\
\hline Ocalea puncticollis Mulsant \& REY, 1875 & O87 \\
\hline Oligota inflata (MANNERHEIM, 1830) & O87 \\
\hline Placusa pumilio (GRAVENHORST, 1802) & O87 \\
\hline \multicolumn{2}{|l|}{ Oxytelinae } \\
\hline Anotylus schatzmayri (KocH, 1937) & Br39 \\
\hline $\begin{array}{l}\text { Carpelimus parvulus (Mulsant \& REY, } \\
\text { 1861) }\end{array}$ & O87 \\
\hline Ochthephilus aureus (FAUveL, 1871) & F51, Ho63 \\
\hline \multicolumn{2}{|l|}{ Steninae } \\
\hline Stenus cyaneus BAUdi di SELVE, 1848 & O87 \\
\hline Stenus morio GravenHORST, 1806 & O87 \\
\hline Stenus nanus StePHens, 1833 & O87 \\
\hline \multicolumn{2}{|l|}{ Scydmaeninae } \\
\hline Leptocharis creticus (PIC, 1903) & Pi03 \\
\hline \multicolumn{2}{|l|}{ Paederinae } \\
\hline Astenus bimaculatus (ERICHSON, 1840) & Sz64 \\
\hline Astenus immaculatus StePhens, 1833 & Sz64 \\
\hline Astenus melanurus (KüsTER, 1853) & Sz64 \\
\hline Oedichirus rubronotatus PIC, 1903 & App, Pi03 \\
\hline Oedichirus terminatus ERICHSON, 1843 & Ko36 \\
\hline Pseudobium labile (ERICHSON, 1840) & Kz58a, O87 \\
\hline Sunius bicolor (OLIVIER, 1795) & Sz64 \\
\hline \multicolumn{2}{|l|}{ Staphylininae } \\
\hline Gabrius trossulus (NoRDMANN, 1837) & Kz58a, O87 \\
\hline Leptacinus pusillus (STEPHENs, 1833) & Sz64 \\
\hline Leptacinus sulcifrons (STEPHENS, 1833) & Sz64 \\
\hline Ocypus aeneocephalus (DE GEER, 1774) & $\mathrm{He} 84, \mathrm{O} 87$ \\
\hline Quedius coxalis KrAATZ, 1858 & O87 \\
\hline Quedius fulgidus creticus MAŘAN, 1935 & Mn35 \\
\hline Quedius fuliginosus (GRAVENHORST, 1802) & Kz58a, O87 \\
\hline Quedius plancus ErICHson, 1840 & O87 \\
\hline Quedius suturalis Kiesenwetter, 1845 & O87 \\
\hline Xantholinus rufipes LucAs, 1846 & Bo14 \\
\hline
\end{tabular}

\subsection{Ecology}

The known fauna is primarily composed of species inhabiting the leaf litter and upper layers of the soil in terrestrial habitats (forests, bushland, phrygana, grassland, etc.) at altitudes from near sea-level to approximately $2000 \mathrm{~m}$; these alone account for approximately $47 \%$ of the diversity (Fig. 3). Another $18.5 \%$ and $4.5 \%$ are contributed by wetland (inhabitants of stream banks, pond margins, etc.) and coastal species (inhabitants of seaweed, debris, sand, 
and gravel on beaches), respectively. Species inhabiting various decaying matter (dung, compost, carrion, etc.) account for $17 \%$ of the fauna, while corticolous $(<2 \%)$ and fungicolous elements $(1 \%)$ are evidently underrepresented. Endogean species (2\%) and species inhabiting deep soil layers, but not strictly endogean (5\%), which are mostly anophthalmous or microphthalmous and practically exclusively collected by soil washing, together make up approximately $7 \%$ of the diversity. Fifteen species (4\%) are associated with ants of the genera Messor Forel, 1890, Tetramorium MAYr, 1855, and Lasius FABricius, 1804. For more details regarding the ecological composition of the endemic fauna see section 3.6.

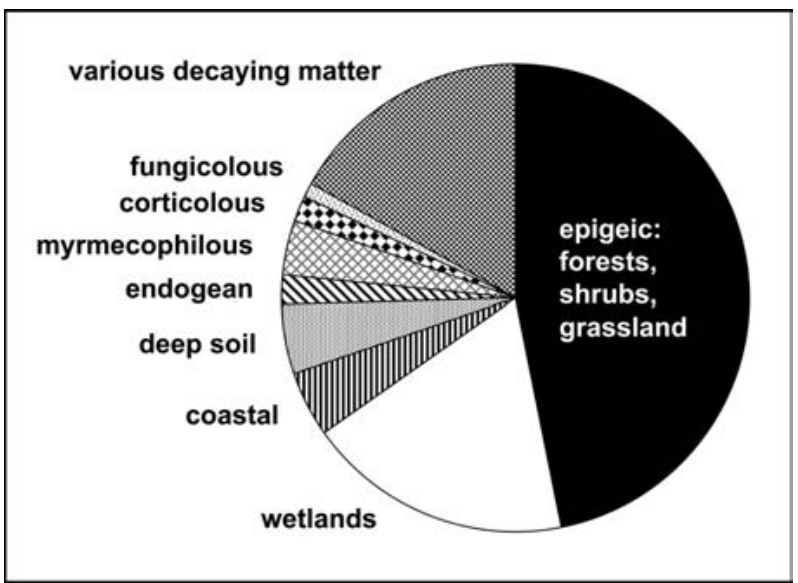

Fig. 3: Ecological composition of the Staphylinidae fauna of Crete (named species).

\subsection{Endemic fauna}

Based on currently available evidence, as many as 111 of the named and at least ten of the unnamed species are endemic to Crete. Thus, the endemics account for nearly $30 \%$ of the total diversity and the rate of endemism is higher than in any other Mediterranean island (see section 3.7).

Most of the named endemic species belong to the Aleocharinae (35 species; $31.5 \%)$, Scydmaeninae (23; $21 \%$ ), and Pselaphinae $(20 ; 18 \%)$, these three subfamilies alone accounting for nearly two-thirds of the endemic fauna of Crete (Fig. 4). Other subfamilies with substantial numbers of endemics (all of them named) are the Paederinae (11 species; $10 \%$ ), followed by the Leptotyphlinae (seven species; $6 \%$ ), the Staphylinidae (six species; $5.5 \%$ ), and the Omaliinae (five species; $4.5 \%$ ). The remaining four species (3\%) belong to the Proteininae, Tachyporinae, Osoriinae, and Steninae (one species each).

The genera including the greatest number of endemics are Cephennium Müller \& Kunze, 1822 of the Scydmaeninae (twelve named species), Geostiba Thomson, 1858 of the Aleocharinae (nine named species, all of them in the subgenus Sipalotricha Scheerpeltz, 1931), Afropselaphus JEAnNEL, 1950 of the Pselaphinae (four named and at least two unnamed), Myrmecopora SAULCY, 1865 of the Aleocharinae (five named species, all of them in the nominal subgenus), Stenichnus Thomson, 1859 of the Scydmaeninae (five named species), and Amauronyx Reitter, 1882 and Tychus LeAch, 1817 of the Pselaphinae (each with four named species).

Nearly half $(49.5 \%)$ of the named endemic species are inhabitants of the litter layer and upper soil strata of forests, as well as bush-, shrub-, and grasslands at lower and intermediate altitudes (Fig. 5). Seven (6.3\%) species are confined to open habitats at higher altitudes (usually $1300 \mathrm{~m}$ or higher); most of them were exclusively found at margins of snow fields. Fourteen wetland species (inhabitants of stream banks, pond margins, swamps, etc.) account for $12.6 \%$ of the named endemic fauna.

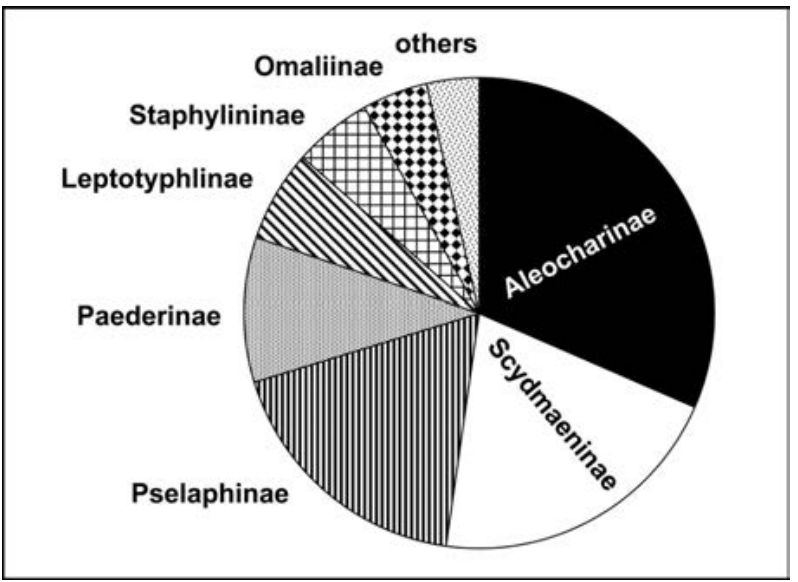

Fig. 4: Composition of the endemic fauna of Crete by subfamilies (named species).

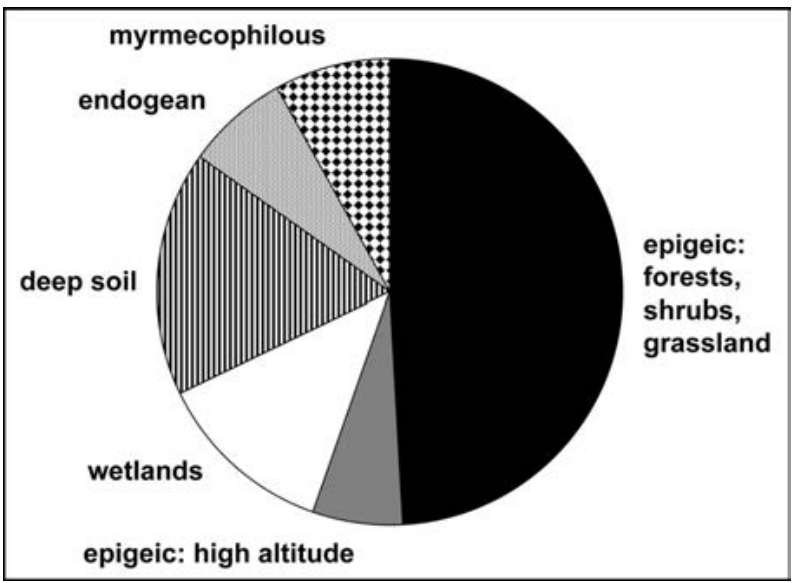

Fig. 5: Ecological composition of the endemic fauna of Crete (named species).

Nine species ( $8 \%$ ) are associated with ants of the genera Messor (five species), Tetramorium (two species), and Lasius (two species). Approximately one-fourth (24.3\%) of the endemic species are either endogean (8 species; $7.2 \%$ ) or inhabitants of deeper soil layers (19 species; $17.1 \%$ ) and were found exclusively (endogean) or almost exclusively by soil washing.

More than half of the named endemic fauna (67 species; $60 \%$ ) can be considered local endemics. The distributions of these species are confined to the extreme west of Crete (three species), the Lefka Ori and envi- 
rons (20 species), most of the western portion of Crete (including the Lefka Ori and the extreme west) (four species), the Psiloritis range (ten species), the Dikti range and environs (including Selena Oros) (14 species), the Thripti range and environs (ten species), the extreme east of Crete (environs of Sitia and Zakros) (four species), or most of the eastern portion of Crete eastwards to Dikti Oros (two species).

\subsection{Evolution of the endemic fauna}

In principle, two major speciation scenarios can be assumed to explain the remarkable diversity of Cretan endemics. One of them is vicariance with mainland species, i.e., a separation of gene pools either resulting from Crete losing its connection to the mainland more than five million years ago or resulting from colonization events and subsequent speciation since then. Molecular studies and a fairly accurate molecular clock would be required to decide which of these two options are realized. The second principle speciation scenario is in-situ radiation on the island, resulting in monophyletic groups of endemic species. In fact, based on morphological characters, as many as 18 such monophyletic lineages representing a total of $59(53 \%)$ of the named species were identified in five subfamilies and 17 genera: Afropselaphus, Amauronyx, Faronus AubÉ, 1844, and Tychus of the Pselaphinae (each with one lineage including three species), Bellatheta Roubal, 1928 (one lineage, two species), Geostiba (one lineage, nine species), Myrmecopora (one lineage, five species associated with Messor spp.), Oxypoda Mannerheim, 1830 (one lineage, two species), and Tectusa Bernhauer, 1899 (one lineage, three species) of the Aleocharinae, Cephennium (two lineages with six and five species, respectively) and Stenichnus (one lineage, two species) of the Scydmaeninae, Cretotyphlus Assing, 2019 (one lineage, three species), Allotyphlus Colffait, 1955 (one lineage, two species), and Kenotyplus Coiffait, 1957 (one lineage, two species) of the Leptotyphlinae, Astenus DejEAN, 1833 (one lineage, two species associated with Tetramorium spp.), Leptobium CASEy, 1905 (one lineage, two species), and Sunius Stephens, 1829 (one lineage, two species) of the Paederinae. The remaining 53 (47\%) named endemics have their closest relatives on the mainland (Greece, Turkey) and/or other East Mediterranean islands and are evidently the result of vicariance with these species, e.g., Sepedophilus creticus with S. nigripennis (Stephens, 1832), Atheta cretica with other species of the A. elongatula group, Drusilla cretica with D. meridiana (FAUVEL, 1900) and D. taygetana Assing, 2005, Pronomaea wunderlei with P. picea Heer, 1841, Medon beroni with other species of the M. fusculus group, $M$. cerrutii with other species of the $M$. petrochilosi group, Pseudobium creticum with P. hellenicum Assing, 2006, Quedius sigwalti with Q. umbrinus ERICHSON, 1839, Xantholinus erinaceus with X. rufipennis ERICHSON, 1839, and $X$. minos with X. graecus KRAATZ, 1858.

\subsection{Comparison with other Mediterranean islands}

A meaningful assessment of the fauna of Crete also requires a comparison with the faunas of other Mediterranean islands. Nine islands were selected: the four islands of similar (Corsica, Cyprus) or significantly larger size (Sardinia, Sicily), the best studied Ionian island (Corfu), and four Aegean islands (Rhodos, Lesbos, Samos, Karpathos) (Tab. 4). The Staphylinidae of the Aegean islands Chios, Ikaría, and Kos have been studied, too (Assing 2015d, 2016b, 2017d), but are too small and poor in diversity to serve as meaningful reference.

In short, the geological history relevant for the diversities and endemism of the islands shown in Tab. 4 may be characterized as follows:

According to recent evidence, Cyprus is an oceanic island, whose major uplift took place since the miocene and which never had a real land bridge to the mainland (Hadjisterkotis 2012). Corfu and the Aegean islands Samos and Lesbos have been separated from the mainland only since the pleistocene (Assing 2016b, 2017c, Triantis \& Mrlonas 2009), whereas the last connection of Rhodos and Karpathos to the mainland dates back to the pliocene (Assing 2013d, 2016a, Triantis \& Mylonas 2009). Corsica and Sardinia are situated on the same microplate and were once part of what is the northeastern Iberian Peninsula today. There are two main hypotheses on the time of the separation of the CorsicaSardinia microplate from the Iberian plate and the history that followed. According to the traditional scenario, the separation began in the Oligocene, there never was a land bridge since, and the disjunction of the two islands started 15 million years ago and was completed nine million years before present. A more recent hypothesis suggests that the Corsica-Sardinia microplate broke away from the Iberian plate 20-24 million years ago and remained connected to Paleo-Europe by a land-bridge (to what is the Maritime Alps and the Ligurian Apennines today) until the Pliocene (approximately 5 million years before present) (KeTMAIER et al. 2006). Thus, irrespective of which of the two scenarios is favoured, it can be assumed that Corsica and Sardinia have been separated from the Iberian mainland for at least 20 million years and that the endemic flora and fauna primarily derived from that present at the time of the separation from the Iberian plate. (It should be noted that neither of the two hypotheses outlined above plausibly explains the distribution of the paederine genus Scotonomus Fauvel, 1873, which exclusively includes blind endogean species and whose distribution is confined to Sardinia and the Central Apennines.) Unlike Corsica and Sardinia, Sicily had a land-bridge connection to Calabria during the pleistocene glacial maxima (ANTONIOLI et al. 2012).

A comparison of the diversities of the staphylinid faunas of the Mediterranean islands is somewhat problematic, since they greatly differ regarding the time and intensity of entomological study. Corsica, Sardinia, and Sicily 
have frequently been visited by numerous entomologists and have even had researchers living on these islands. In the East Mediterranean, only Corfu can be considered fairly well studied. Other East Mediterranean islands, by contrast, had received only little attention until very recently. It follows that there is considerable bias owing to different study intensities particularly regarding the figures for total species diversity. Without doubt, the number of Staphylinidae species known from these islands (including Crete) could still be increased significantly by sampling habitats such as decaying matter of all kinds (compost, dung, rotting wood, etc.), nests of subterranean mammals and birds, mushrooms, etc., as well as by using additional methods such as flight-interception traps and pitfall traps. So far, the focus has been on the endemic epigeic fauna and sampling mainly relied on sifting, hand-collecting (turning stones), floating (riparian habitats), and soil-washing (Crete only). On the other hand, the sampling bias is much less pronounced when considering the endemic faunas of the compared islands, at least the epigeic endemic faunas. Several Aegean islands are known to host some additional undescribed species of Pselaphinae and Scydmaeninae and a few species may still be discovered in the future, but this is unlikely to have a significant impact on the general trends shown in Tab. 4.

Unsurprisingly, overall diversity of the Cretan fauna is significantly greater than that of the much smaller Aegean islands (Tab. 4). Yet, the difference is not as great as could be expected based on area alone. The known fauna of Lesbos, for instance, an island with an area of approximately one-fifth of that of Crete, is composed of 201 species and that of Samos, an island with an area of only one-twentieth of that of Crete, includes 157 named plus additional unnamed species. Unlike Crete, however, Lesbos and Samos were connected to the Anatolian mainland during the latest pleistocene glacial maxima and are separated from the nearest mainland by narrow straits only a few kilometres wide, which do not form an effective barrier for flying insects and thus allow for frequent and continuous colonization from the mainland. The greater diversity of the Cretan fauna in comparison to that of the larger Cyprus, despite the greater distance to the mainland, may be explained by the significantly greater topological diversity of Crete, the much richer endemic fauna, and the assumption that Cyprus is an oceanic island. On the other hand, the Staphylinidae fauna of Crete is significantly less diverse than the faunas of the three similarly large or much larger Mediterranean islands Corsica, Sardinia, and Sicily. To some extent, this can be attributed to the sampling bias mentioned above.

The current fauna of Sicily and particularly those of the geological old Corsica and Sardinia can be assumed to be largely composed of elements originating from the original faunas at the time of separation from the mainland. In addition, Sicily is separated from the South Italian mainland only by the narrow Strait of Messina and has had a land connection even in the late pleistocene. Crete, on the other hand, has had a volatile history of partial submergence and uplift since its separation from the mainland, suggesting that much of its presentday non-endemic fauna is the result of colonization. Considering its isolated geographic position, it can be assumed that the sea separating it from the mainland forms an effective barrier particularly for species with low dispersal power and successful colonization events are rather unlikely.

The lower total diversity of the Cretan fauna compared to that of the much smaller Ionian island Corfu, too, is primarily explained by the degree and history of isolation. Corfu had a land connection to the mainland during the last glacial maximum and is today separated from it by only few kilometres. It addition, this island is characterized by remarkable habitat diversity including extensive wetlands, has frequently been visited by entomologists, and has been studied with a variety of methods.

A comparison of the endemic fauna of the islands reveals that endemic species are not only significantly more numerous, but also form a much larger proportion of total diversity (nearly one-third) in Crete than in other East Mediterranean islands. The larger Cyprus hosts less than one-fourth as many endemics, which account for only $8.3 \%$ of its total diversity. This discrepancy may be explained by the oceanic origin of Cyprus, its shorter distance to the mainland, and by the topology: Cyprus only has one major mountain range (Troodos) in the west and a significantly lower mountain chain (highest elevation at little more than $1,000 \mathrm{~m}$ ) in the northeast, conditions rendering in-situ radiation less likely. Despite its much greater total diversity, the fauna of Corfu includes only few endemic species, all of them either hypo- or endogean. The other East Mediterranean islands shown in Tab. 4 are all significantly smaller and, except for Karpathos, separated from the Turkish mainland only by very narrow straits. The relatively high proportions of endemics in the faunas of the small islands Samos and Karpathos can be explained by the presence of two major mountains (Samos) and geographic isolation (Karpathos).

The diversity of the endemic fauna of Crete clearly exceeds that of the three times larger Sicily, which is primarily explained by geological history of Sicily and its vicinity to the mainland. Moreover, Sicily has had a long history of intense cultivation by humans, suggesting that part of its original endemic fauna may now be extinct.

A comparison with the faunas of Corsica and Sardinia, on the other hand, reveals that they host significantly more endemic species than Crete. True, these islands have had a long history of entomological field work and have been subject to more intense sampling than Crete, but it seems unlikely that, even with increased efforts, the number of endemic species known from Crete will ever add up to 140 (Sardinia), let alone 186 (Corsica). Nevertheless, the relative representation of endemics in the Staphylinidae 
fauna of Crete is significantly greater than in Sardinia (23\%) and Corsica (24\%). It seems noteworthy that the endogean fauna accounts for more than half of the endemic fauna in Corsica and for nearly half (40\%) in Sardinia, as opposed to only approximately $7 \%$ in Crete. The pronounced north-south gradient in the diversity of the endogean fauna from Corsica to Sicily is remarkable, but wanting a convincing explanation. In any case, the presence of a speciose endogean fauna in Corsica and Sardinia would support the hypothesis that the faunas of these islands are largely composed of elements originating from the original fauna at the time of the separation from the mainland rather than from colonization. Regarding the epigeic endemic fauna, Crete is clearly more diverse than Corsica, more diverse even than the three times larger Sardinia.

Tab. 4: Total diversity and endemism of the Staphylinidae of Crete and selected other Mediterranean islands.

Figures for islands other than Crete are based on

a) SCHüLKe \& SMetana (2015) including additions up to the end of 2017: Cyprus; endemics of Corsica, Sardinia, and Sicily;

b) Assing (2013d, 2017d): Rhodos; Assing (2016a): Karpathos; Assing (2016b): Lesbos; Assing (2017c, d): Samos; Assing et al. (2018): Corfu (unnamed species neglected);

c) Ciceroni et al. (1995), Angelini et al. (1995), Zanetti (2011), Assing (2014c): total diversity of Sardinia and Sicily (doubtful records neglected);

d) Tronquet et al. (2014) and Tronquet (pers. comm.): total diversity of Corsica.

The species total given for the islands is exclusively based on named species. Endogean species include all Leptotyphlinae and Osoriinae, as well as all Mayetia Mulsant \& Rey, 1875 (Pselaphinae), Octavius Fauvel, 1873 (Euaesthetinae), and Scotonomus FAUvel, 1873 (Paederinae). Species whose habitat (endogean or epigeic) is transitional or uncertain (e.g., Cephennium spp., Typhlocyptus SAULCY, 1878) and possibly endogean species of taxa other than those mentioned above are treated as epigeic.

\begin{tabular}{|c|c|c|c|c|c|c|c|c|c|c|}
\hline & Crete & Cyprus & Corfu & Samos & Lesbos & Rhodos & Karpathos & Corsica & Sardinia & Sicily \\
\hline area $(\mathrm{km} 2)$ & 8,336 & 9,251 & 585 & 477 & 1,633 & 1,408 & 302 & 8,680 & 24,090 & 25,426 \\
\hline $\begin{array}{l}\text { distance to } \\
\text { mainland }(\mathrm{km})\end{array}$ & 100 & $\sim 65$ & 3 & 3 & 11 & 21 & 93 & $\sim 80$ & $\sim 200$ & 3 \\
\hline species total & 397 & 326 & 446 & 157 & $201^{1)}$ & $134^{1)}$ & $69^{1)}$ & $\sim 785$ & 615 & 605 \\
\hline \multicolumn{11}{|c|}{ endemics } \\
\hline no. species & 111 & 27 & $7^{2)}$ & $17^{1)}$ & $11^{1)}$ & $11^{1)}$ & $9^{1)}$ & 186 & 140 & 80 \\
\hline$\%$ species total & 28 & 8.3 & 1.6 & 10.8 & 5.5 & 8.5 & 13 & 24 & 22.8 & 13.2 \\
\hline endogean & 8 & - & 6 & - & - & 1 & - & 106 & 56 & 4 \\
\hline non-endogean & 103 & 27 & 1 & 17 & 11 & 10 & 9 & 80 & 84 & 76 \\
\hline undescribed & $\geq 10$ & - & - & 6 & 2 & 3 & 3 & - & - & - \\
\hline Omaliinae & 5 & 2 & & & & & & 7 & 3 & 4 \\
\hline Proteininae & 1 & 1 & & & & & & 1 & & 1 \\
\hline Pselaphinae & 20 & 5 & 3 & 7 & 7 & 1 & 2 & 50 & 43 & 25 \\
\hline Phloeocharinae & & & & & & & & 1 & 1 & \\
\hline Tachyporinae & 1 & & & & & & & 1 & & 2 \\
\hline Aleocharinae & 35 & & 2 & 2 & & 1 & 1 & 19 & 12 & 17 \\
\hline Scaphidiinae & & & & & & & & & 1 & 1 \\
\hline Osoriinae & 1 & & & & & & & 4 & 3 & 2 \\
\hline Oxytelinae & & 1 & 2 & & & & & & 2 & 1 \\
\hline Steninae & 1 & 2 & & & & & & 1 & & \\
\hline Euaesthetinae & & & & & & & & 13 & 4 & \\
\hline Scydmaeninae & 23 & 3 & 2 & 5 & 3 & 6 & 5 & 17 & 16 & 9 \\
\hline Leptotyphlinae & 7 & & 6 & & & 1 & & 68 & 24 & 2 \\
\hline Pseudopsinae & & 1 & & & & & & & & \\
\hline Paederinae & 11 & 8 & 1 & 3 & 1 & 2 & 1 & 3 & 30 & 7 \\
\hline Staphylininae & 6 & 4 & 1 & & & & & 1 & 1 & 9 \\
\hline
\end{tabular}

Footnotes: ${ }^{1)}$ Named and unnamed species; ${ }^{2)} 10$ addititional species are currently known only from Corfu, but of doubtful status or unlikely to be endemic. 


\subsection{Additional records}

\section{Myllaena cf. graeca KRAATZ, 1858}

Material examined: Greece: Crete: $2 \sigma^{\star} o^{\star}$, “Creta", leg. v. Oertzen (NHMW).

The original description of M. graeca is based on an unspecified number of syntypes from "Zante (v. Kiesenwetter), Creta (Zebe)" (KrAATZ 1858a). Both of the above specimens are teneral males. They belong to a species group that includes at least three species distinguishable only based on the shape of the spermatheca, or at least on mature specimens (Assing 2018d).

\section{Stenus assequens REY, 1884}

Material examined: Greece: Crete: $1 o^{\pi}$ [det. Puthz], Lassithi, 14.VII.1970, leg. Senglet (MHNG); 1 ㅇ, "Dedmation”, leg. Cerruti (ZIUR); 1 ex. [det. Puthz], locality not specified, leg. Paganetti (NHMW).

\section{Stenus ganglbaueri BeRnHAUER, 1905}

Material examined: Greece: Crete: $2 o^{\star} o^{*}, 1$ ㅇ [det. Puthz], Minotha, swamp, 29.III.1970, leg. Irmler (cIrm); 1 \% [det. Puthz], Samaria, gravel bank, 3.IV.1970, leg. Irmler (cIrm); 4 우 우 [det. Puthz], locality not specified (BMNH, SDEI).

\section{Stenus pallitarsis StePHENS, 1833}

Material examined: Greece: Crete: $1 \mathrm{o}^{\mathrm{A}}, 3$ ㅇ ㅇ [det. Puthz], Pirgos, 29.IV.1971, leg. Malicky (SMNS).

\section{Stenus picipes picipes StEPHENS, 1833}

Material examined: Greece: Crete: numerous specimens (number not specified) [det. Puthz], Moni Venius, 23.V.1977, leg. Malicky (SMNS); specimen number not specified [det. Puthz], numerous localities (MNB, SMF, SNM, ZMUC).

\section{Leptomastax bipunctata ReITTER, 1881}

Material examined: Greece: Crete: 2 ㅇ ㅇ [det. Meybohm], Chania, S Vrises, $35^{\circ} 21^{\prime} 41^{\prime \prime} \mathrm{N}, 24^{\circ} 11^{\prime} 44^{\prime \prime E}, 130 \mathrm{~m}$, calcareous olive grove, 8.IV.2012, leg. Germann (NHMB).

Unlike the other Leptomastax species recorded from Crete, L. bipunctata is not endemic, its distribution including Turkey and the Balkans from the Pelopónnisos northwards to Croatia. It appears likely that the species was introduced to Crete recently, as is suggested by the local occurrence in Crete and by the observation that other non-endemic Scydmaeninae known from Crete are widespread in the island (Меувонм pers. comm.).

\section{Paederus littoralis GRAVENHORST, 1802}

Material examined: Greece: Crete: 1 ㅇ, Kourna Lake, 25.II.1997, leg. Schmidt (cAss).

\section{Gyrohypnus fracticornis (MüLleR, 1776)}

Material examined: Greece: Crete: 1 ex., Rethimnon, Ideon Andron, 26.VI.1989, leg. Sama (MNB); 1 ex.,

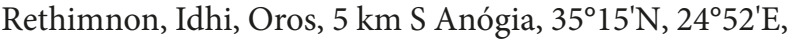
1150 m, 28.IV.1995, leg. Lange \& Ziegler (SDEI).

\subsection{Notes on some species}

Cousya spec. nov.

Material examined: 1 ㅇ, Psiloritis, ca. 8 km SSW Zoniana, $35^{\circ} 13^{\prime} 45^{\prime \prime} \mathrm{N}, 24^{\circ} 48^{\prime} 10^{\prime \prime E}, 1730 \mathrm{~m}$, litter and soil near margins of snowfields sifted, 15.IV.2014, leg. Wunderle (cAss).

Comment: The above female clearly represents an undescribed species. A description is refrained from for want of males.

\section{Lesteva brondeeli LOHSE \& STEEL, 1961}

This species was previously listed as an endemic of Crete (Assing 2013a, 2015a). However, Puthz (1979) recorded the species from Naxos, so that it can no longer be considered endemic.

\section{Proteinus creticus Assing, 2004}

This species was originally described from Crete, where it was repeatedly recorded again, partly in large numbers (Assing 2004b, 2013a, 2015a). The species was subsequently reported from West Anatolia (Anlaş \& TEzCAN 2008), but this record appears highly doubtful and requires confirmation. For the time being, P. creticus is considered an endemic of Crete.

\section{Phloeocharis longipennis FAUVEL, 1875}

Phloeocharis subtilissima var. hummleri BeRnhAuer, 1915: 69; syn. nov.

Phloeocharis hummleri was originally described as a variety of $P$. subtilissima, based on an unspecified number 
of syntypes from "Kreta" (Bernhauer 1915). According to the original description, it is distinguished from P. subtilissima only by longer elytra and the presence of a palisade fringe at the posterior margin of the abdominal tergite VII. ScheERPELTz (1931) regarded the taxon as a distinct species. An examination of recently collected material (including a male) from Crete, which is in perfect agreement with the original description of $P$. hummleri, revealed that it is conspecific with $P$. longipennis FAUvEL, 1875. Since there is little doubt that the same is true of the type material of $P$. hummleri, this name is placed in synonymy with P. longipennis. The previously known distribution of P. longipennis ranged from the Greek Aegean islands Lesbos and Samos across Turkey to the Middle East (Lebanon, Syria, Israel) (Assing 2004c, 2005c, Schülke \& Smetana 2015). The record of P. hummleri from Hungary by Tóтн (1982) most likely refers to P. subtilissima MANnerheim, 1830.

\section{Dinusa cretica Assing, 2013}

This species had been considered an endemic of Crete (Assing 2013a, 2015a), until it was recently also discovered in Karpathos (Assing 2016a) and Samos (Assing 2017c).

\section{Atheta (Datomicra) nigra (KRAATZ, 1856)}

Atheta (Microdota) biroi Scheerpeltz, 1964: 306 ff.; syn. nov.

The original description of Atheta biroi is based on " 1 ơ, 1 ㅇ, Typen, 76 Ex., Paratypen" from "Herakleion" (Scheerpeltz 1964). According to Jürgen Vogel (Görlitz), who revised type specimens, this material is conspecific with the widespread and common A. nigra. Hence the synonymy proposed above.

\section{Genus Hydrosmecta Thomson, 1858}

This genus is currently in a state of taxonomic confusion. Some West Mediterranean species were recently revised and a synopsis of the species recorded from France was provided by Tronquet (2016). However, this article focuses on a regional fauna and does not include numerous additional described species reported from other regions of the West Palaearctic. Hydrosmecta species are typical inhabitants of gravel banks, usually of rivers and streams. They are active flyers and can consequently be assumed to be widespread.

As many as six species were recorded from Crete. Only two of them, $H$. longula and $H$. fluviatilis, were identified. A third species was tentatively determined as $H$. cf. perpusilla; this species was recorded also from Samothraki and a locality in Fokis, Greek mainland (unpublished). Only one of the three remaining species is conspicuous enough to rule out a previous description based on the diagnoses available in the literature and to justify a description (see H. insularum in part II of the monograph). The two other species (see Tab. 2) may be undescribed, but remain unnamed. Neither of them is endemic to Crete. Hydrosmecta sp. 1 (= Hydrosmecta sp. in Assing (2015a)) was collected also in two localities in Fthiotis and Fokis, Greek mainland, and Hydrosmecta sp. 2 in the Greek mainland (several localities) and Cyprus (unpublished records).

\section{Hydrosmecta fluviatilis (KRAATZ, 1854)}

Material examined: Greece: Crete: 42 exs., ESE Perama, N Garazo, 35 $21^{\circ} 05^{\prime \prime} \mathrm{N}, 24^{\circ} 47^{\prime} 01^{\prime} \mathrm{E}, 130 \mathrm{~m}$, stream bank, gravel floated, 4.IV.2012, leg. Assing (cAss). Lesbos: $10^{7}$, 1 , $14 \mathrm{~km}$ WNW Mytilini, S Lambou Mili, 3907'51"N, $26^{\circ} 23^{\prime} 55^{\prime \prime} \mathrm{E}, 50 \mathrm{~m}$, stream bank, hand collected and floated from gravel, 20.III.2016, leg. Assing \& Hetzel (cAss); $1 o^{*}, 6.5 \mathrm{~km}$ ENE Vatera, Stavros, Vourkos river, $39^{\circ} 02^{\prime} 21^{\prime \prime N}, 26^{\circ} 16^{\prime} 09^{\prime \prime} \mathrm{E}, 50 \mathrm{~m}$, river bank with gravel, 27.III.2016, leg. Assing \& Hetzel (cAss). Samothraki: $2 \sigma^{\top} o^{\star}$, W Lakkoma, $40^{\circ} 25^{\prime} 57^{\prime \prime} \mathrm{N}, 25^{\circ} 30^{\prime} 54^{\prime \prime} \mathrm{E}, 20 \mathrm{~m}$, river bank, gravel floated, 9.IV.2019, leg. Assing (cAss); 1 , same data, but 16.IV.2019 (cAss); $10^{\star}$, SSE Ano Meria, $40^{\circ} 26^{\prime} 21^{\prime \prime} \mathrm{N}, 25^{\circ} 41^{\prime} 39^{\prime \prime} \mathrm{E}, 5 \mathrm{~m}$, stream gravel floated, 14.IV.2019, leg. Assing (cAss); $220^{\star} o^{\star}, 35$ 우, SE Ano Meria, $40^{\circ} 26^{\prime} 52^{\prime \prime} \mathrm{N}, 25^{\circ} 41^{\prime} 46^{\prime} \mathrm{E}, 5 \mathrm{~m}$, stream gravel floated, 14.IV.2019, leg. Assing (cAss); 1 ㅇ, SE Kamariotissa, $40^{\circ} 26^{\prime} 48^{\prime \prime} \mathrm{N}, 25^{\circ} 29^{\prime} 53^{\prime \prime} \mathrm{E}, 40 \mathrm{~m}$, bank of temporary stream, gravel floated, 16.IV.2019, leg. Assing (cAss); 1 ㅇ, E Therma, $40^{\circ} 30^{\prime} 03^{\prime \prime} \mathrm{N}, 25^{\circ} 37^{\prime} 00^{\prime \prime} \mathrm{E}, 5 \mathrm{~m}$, stream bank, gravel floated, 17.IV.2019, leg. Assing (cAss). Mainland: 10 exs., ca. $30 \mathrm{~km} \mathrm{SW}$ Lamia, bank of Inachos river near Perivoli, 3849'31"N, 2204'58"E, 470 m, 5.IV.2001, leg. Assing \& Wunderle (cAss); $10^{\star}, 1$ ㅇ , Lamia env., Kamena Vourla, dry stream bed, 1.VIII.1987 (cAss). Pelopónnisos: $1 \sigma^{\star}$, Sparta, stream bank, debris, 2.IV.1986, leg. Assing (cAss).

The above material from Crete was recorded as Hydrosmectasp. by Assing (2013a), that from Lesbos as Hydrosmecta sp. 1 by Assing (2016b). According to SchülKe \& SMETANA (2015), H. fluviatilis was previously unknown from Greece.

\section{Hydrosmecta longula (HEER, 1839)}

Material examined: Greece: Crete: 28 exs., ESE Perama, N Garazo, 35² $21^{\prime} 05^{\prime \prime N}, 24^{\circ} 47^{\prime} 01^{\prime \prime E}, 130 \mathrm{~m}$, stream bank, gravel floated, 4.IV.2012, leg. Assing (cAss); 23 exs., WSW Agios Nikolaos, Katharo plateau, $35^{\circ} 08^{\prime} 14^{\prime \prime}$, $25^{\circ} 34^{\prime} 15^{\prime \prime E}$, $1110 \mathrm{~m}$, stream bank with gravel, 11.IV.2014, leg. Assing (cAss).

The above material was recorded as Hydrosmecta sp. by Assing (2013a, 2015a). 


\section{Oligota muensteri BERNHAUER, 1923}

The material listed in Tab. 1 represents the first records from Greece.

\section{Phytosus balticus KRAATZ, 1859}

Phytosus holtzi Bernhauer, 1935: 48; syn. nov.

Comment: According to the original description, which is based on a unique specimen from East Crete, the holotype is similar to P. balticus, but distinguished by larger body size, longer elytra, longer antennae, darker coloration, and some minor differences in the punctation and shine of the body (Bernhauer 1935). A comparison of specimens collected near Chania (West Crete) (Assing 2015a), which are in agreement with the characters indicated in the description of $P$. holtzi, with material of $P$. balticus from various regions in Europe yielded no evidence suggesting that they should represent distinct species. Phytosus holtzi has never been recorded again since its description. Moreover, the presence of an endemic coastal species in Crete would seem highly unlikely. These observations suggest that the holotype of $P$. holtzi is in fact a specimen of P. balticus of dark coloration and at the upper end of the size range of this species. Hence the synonymy proposed above.

\section{Stichoglossa graeca BERNHAUER, 1905 \\ (Figs 6-10)}

Stichoglossa graeca Bernhauer, 1905: 593 f.

Type material: Lectotype $\sigma^{\star}$, present designation: "Achaja / Lappa / Mir nicht bekannt ?, Genus ??! / graeca Brh. Typus / graeca Brh. det. Bernhauer / Chicago NHMus, M. Bernhauer Collection / Lectotypus o Stichoglossa graeca Bernhauer, desig. V. Assing 2018” (FMNH).

Comment: The original description is based on an unspecified number of syntypes, among them at least one male, from "Griechenland, Achaja" (Bernhauer 1905). The single male syntype located in the Bernhauer collection at the FMNH is designated as the lectotype.

Redescription: Body length 2.3-2.5 mm; length of forebody 1.1-1.2 mm. Habitus as in Fig. 6. Coloration: head blackish-brown; pronotum bright reddish; elytra brown with the humeral angles and the postero-sutural portions diffusely yellowish; abdomen blackish with the anterior segments slightly paler and with the posterior portion of segment VII and all of segments VIII-X yellowish; legs yellowish; antennae dark-reddish with the basal two antennomeres slightly paler; maxillary palpi yellowish with palpomere III weakly infuscate.
Head (Fig. 7) approximately as broad as long; punctation rather sparse and very fine, barely visible in the pronounced microreticulation. Eyes moderately large, slightly shorter than postocular region in dorsal view. Antenna $0.65 \mathrm{~mm}$ long and distinctly incrassate apically; antennomeres IV distinctly transverse, $\mathrm{V}-\mathrm{X}$ of increasing width and increasingly transverse, $\mathrm{X}$ approximately three times as broad as long, and XI of conical shape, slightly longer than the combined length of IX and X.

Pronotum (Fig. 7) 1.2 times as broad as long and 1.2 times as broad as head; posterior angles obtusely marked; punctation fine and moderately dense, barely visible in the pronounced microreticulation.

Elytra (Fig. 7) as long as pronotum; punctation dense, more distinct than that of head and pronotum; interstices with microsculpture, but glossy. Hind wings fully developed.

Abdomen narrower than elytra; anterior impressions of tergites III-V shallow; punctation dense and distinct on tergite III, gradually decreasing in density towards posterior tergites, sparse on tergite VII; interstices with shallow microsculpture and glossy; posterior margin of tergite VII with palisade fringe.

$\sigma^{*}$ : tergite VII (Fig. 8) with small, but distinct median tubercle; tergite VIII (Fig. 8) with small median tubercle posteriorly, posterior margin concave and distinctly serrate; sternite VIII obtusely produced in the middle; median lobe of aedeagus (Figs 9-10) $0.23 \mathrm{~mm}$ long, with broad ventral process (ventral view) and with weakly sclerotized internal structures; parameres approximately $0.3 \mathrm{~mm}$ long and with rather large apical lobe.

q: unknown.

Comparative notes: Stichoglossa graeca is distinguished from other West Palaearctic congeners by its slender (Leptusa-like) habitus, its coloration, and its small body size alone.

Distribution and natural history: The species was previously known only from the type locality in the north of the Pelopónnisos. The male from Crete (Tab. 1) represents the first record of this evidently very rare species since the original description. It was collected by sifting bark of dead chestnut trees at an altitude of $280 \mathrm{~m}$.

\section{Domene stilicina (ERICHSON, 1840)}

Domene losheianum [sic] Bordoni, 1977: 148; syn. nov.

Type material examined: Holotype $\sigma^{\top}$ [teneral]: "Kreta, 19.5.70, Kamanes, leg. Irmler / Holotypus / losheianum [sic] n. sp., Det. A. Bordoni 1976" (cBor).

Comment: The original description of D. lohseiana (emendation of the erroneous original spelling D. "losheianum") is based on a unique male holotype from "Creta, Kamanes, G. A. Lohse leg [sic]" (BordonI 


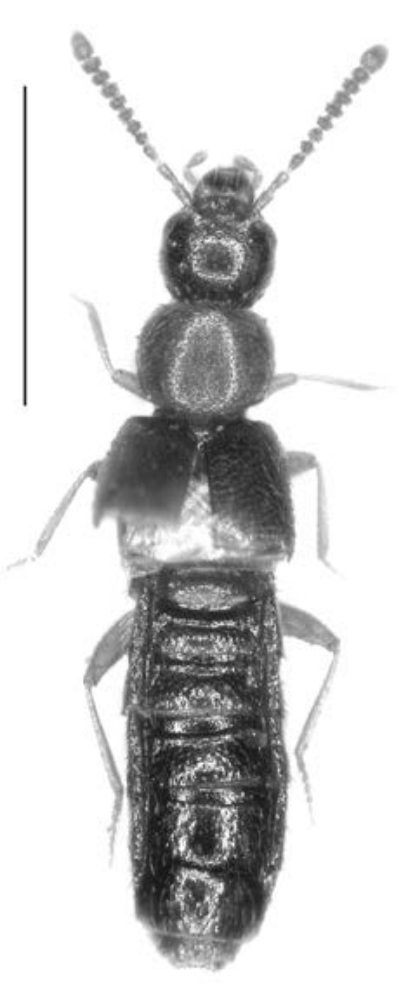

6

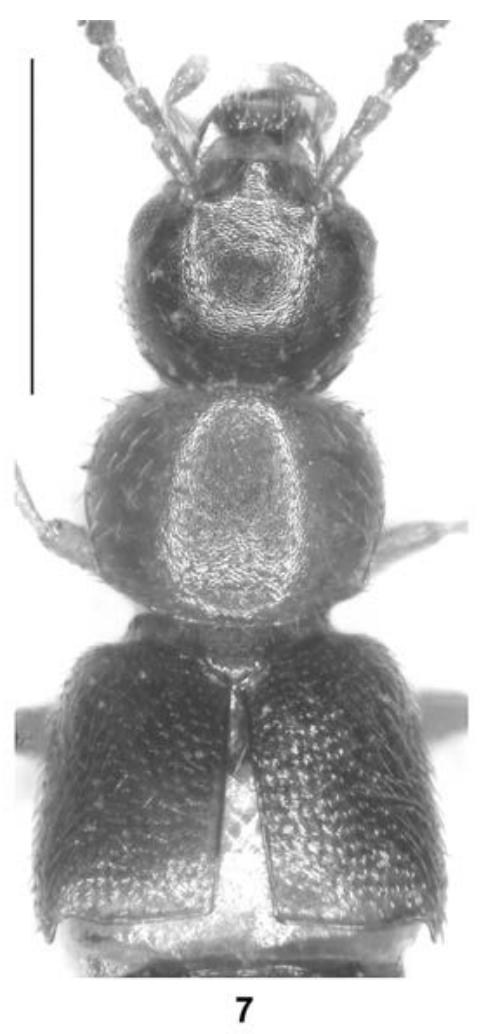

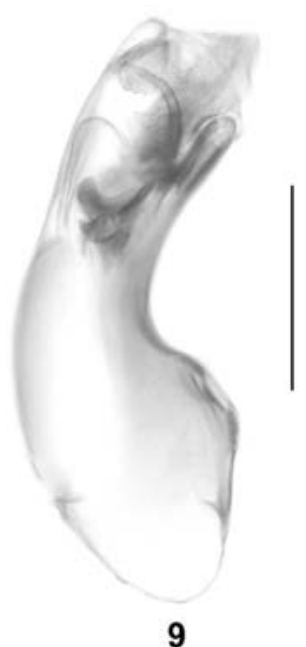
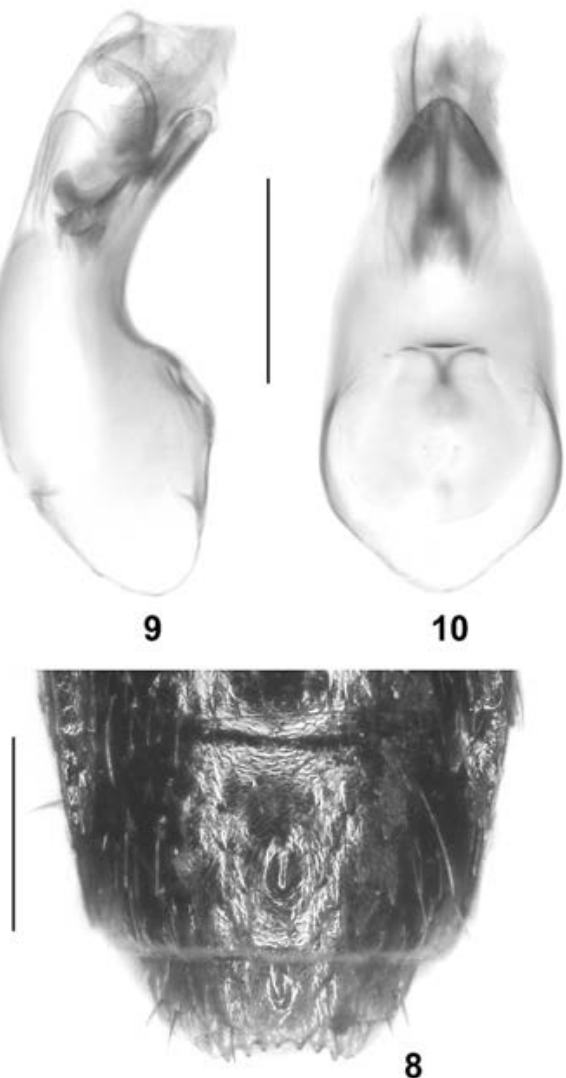

Figs 6-10: Stichoglossa graeca from Crete: habitus (6); forebody (7); male abdominal tergites VII-VIII (8); median lobe of aedeagus in lateral and in ventral view (9-10). Scale bars: 6: $1.0 \mathrm{~mm}$; 7: $0.5 \mathrm{~mm}$; 8: 0.2 $\mathrm{mm}$; 9-10: 0.1 mm.

1977). The true collector is U. Irmler, not G. A. Lohse as indicated by Bordoni (1977). The holotype was examined by Benedikt Feldmann (Münster), who observed that it is conspecific with D. stilicina (FELDMANN e-mail 30.I.2018). The holotype is somewhat teneral, which explains why the aedeagus is deformed and the illustrations in BORDONI (1977) misleading.

\section{Oedichirus rubronotatus PIC, 1903, revalidated}

Oedichirus terminatus var. rubronotatus PIC, 1903: 145. Oedichirus dimidiatus ReITTER, 1906: 263 f.; preoccupied. Oedichirus reitteri BernHAUER, 1908: 33; syn. nov.

Comment: The description of O. rubronotatus, a name originally made available as a variety of O.terminatus ERICHSON, 1843, is based on an unspecified number of syntypes from "Crête" (PIC 1903). The name was treated as infrasubspecific by $\mathrm{KocH}$ (1936) and subsequently regarded as a junior synonym of O. terminatus (SCHÜLKE \& SMetana 2015). Hence, O. terminatus is indicated for Crete in recent catalogues (e.g., Schülke \& SMEtana 2015). According to Horn et al. (1990), the Pic collection is deposited in the natural history museum in Paris. Thus, owing to the current restrictive loan policy of this museum, the type material is inaccessible for scientific study.
The type material of O.terminatus, a species described from Angola, was recently revised by Assing (2019b). This revision revealed that, as was to be expected, O. terminatus is not conspecific with other Oedichirus species examined from the southern West Palaearctic region, so that the previously established synonymy with O. rubronotatus is most likely incorrect.

The original description of $O$. dimidiatus is based on two syntypes from "Zentral-Asien: Baldschuan" and "Kleinasien (Adana)" (Reitter 1906). Bernhauer (1906) noted that the name was preoccupied by O.dimidiatus EpPELSHeIM, 1890 and replaced it with the nomen novum $O$. reitteri. This species has been recorded from Turkey, Cyprus, Tajikistan, and Afghanistan.

Меувонм (2009) discovered that the type locality "Crête" in PIC (1903) does not refer to the Greek island Crete, but to a locality in the environs of Fethiye in the province Muğla, Southwest Turkey. Only two Oedichirus species have been recorded from Turkey, the macropterous $O$. reitteri and the micropterous O. simoni Eppelsheim, 1889. Of these, O. rubronotatus can only be conspecific with O. reitteri for several reasons. PIC (1903) states that the elytra are "largement marqués de roux à l'extrémité" (which is the case also in $O$. reitteri) and since he attributes O. rubronotatus to O. terminatus, it can be inferred that it is winged. Oedichirus simoni, however, has completely blackish (or blackish-blue) elytra, is micropterous, and its distribution is confined to the Middle East 
(from central southern Turkey across Lebanon to Israel). Species of Oedichirus have never been recorded from Crete, despite extensive collecting activity.

These observations all lead to the conclusion that $O$. reitteri is in fact conspccific with, and consequently a junior synonym of, O. rubronotatus.

\section{Pseudolathra quadricollis (FAUvEL, 1875)}

Pseudolathra cretense [sic] BoRdonI, 1986: 387 f.; nov. syn.

Additional material examined.Turkey: $1 \sigma^{\star}$, Osmaniye, $300 \mathrm{~m}, \mathrm{VI} .1968$, leg. Schubert (cAss). Cyprus: $1 \mathrm{o}^{\text {T}}$, Paphos, 6 km N Kedares, 20.VII.2018, leg. Balkenohl (cAss). Greece: Rhodos: $1 \sigma^{\star}$, Kolimbia, Loutani river, 10 m, 14.IV.1994, leg. Frisch (cAss).

The original description of $P$ cretensis is based on a unique male holotype from "Creta, Knossos" deposited in the natural history museum in Prague (BORDONI 1986). This species has never been recorded again. An examination of the four specimens listed in Tab. 1, which are in perfect agreement with the illustrations and description provided by BoRDONI (1986), revealed that they are conspecific with $P$. quadricollis, a species originally described from South Turkey and subsequently reported also from Cyprus, Syria, and Algeria (SCHÜLKE \& Smetana 2015). Consequently, P. cretensis is placed in synonymy with $P$. quadricollis.

\section{Heterothops dissimilis (GRAVENHORST, 1802)}

Comment: A revision of Heterothops material from the East Mediterranean revealed that the existing identification keys are highly erroneous and misleading. Specimens previously reported as H. minutus Wollaston, 1860 not only from Crete, but also from Samos, Ikaría, and Israel (Assing 2013a, 2015a, c, 2017c, Assing \& Feldmann 2012) all belong to $H$. dissimilis. In fact, I have not seen a single specimen of $H$. minutus from the whole of the East Mediterranean region, nor from Central Europe.

\section{Acknowledgements}

This monograph would not have been possible without substantial help from various colleagues. Volker Brachat (Geretsried) and Heinrich Meybohm (Großhansdorf) provided material from several field trips to Crete, identified all the Pselaphinae and Scydmaeninae (except Cephennium spp.), respectively, and critically reviewed the respective sections of the checklist. Paul Wunderle (Mönchengladbach) communicated results of a field trip conducted in 1991. Volker Puthz provided additional data of Stenus species and reviewed the Stenus species in the checklist. Pier Mauro Giachino (Torino), Dante Vailati (Brescia), and Jan Matějíček (Hradec Králové) made material availabe for study. Benedikt Feldmann (Münster) provided information on the synonymy of Domene lohseinana, supplied additional records, and proof-read the manuscript. Jürgen Vogel (Görlitz) provided information on the identity of Atheta biroi and helped with the identification of several Atheta species. Harald Schillhammer (Wien), Michael Schülke (Berlin), and Ivan Löbl (Genève) identified some Philonthus spp., Tachyporinae, and Scaphisoma agaricinum, respectively.

\section{References}

Angelini, F.; Audisio, P.; Castellini, G.; Poggi, R.; Vailati, D.; Zanetti, A. \& Zoia, S. 1995: Coleoptera Polyphaga II (Staphylinoidea escl. Staphylinidae). Fasciculo 47. - In: Minelli, A.; Ruffo, S. \& LA Posta, S. (eds), Checklist delle specie della fauna italiana. - Edizioni Calderini, Bologna: 39 pp.

Anlaş, S. \& Tezcan, S. 2008: New records of Proteininae (Staphylinidae) from Turkey. - Linzer Biologische Beiträge 40 (1): 405-408. - https://www.zobodat.at/ pdf/LBB_0040_1_0405-0408.pdf.

Antonioli, F; Presti, V. L.; Morticelli, M. G.; Mannino, M. A.; Lambeck, K.; Ferranti, L.; BonfiGlio, L.; Mangano, G.; Sannino, G. M.; Furlani, S.; Sulli, A.; Palombo, M. R. \& Canese, S. P. 2012: The land bridge between Europe and Sicily over the past 40 kyrs: timing of emersion and implications for the migration of Homo sapiens. - Rendiconti Online della Società Geologica Italiana, Roma 21: 1167-1169.

Assing, V. 1995: The Palaearctic species of Emplenota Casey, Polystomota Casey, Triochara Bernhauer and Skenochara BERNHAUER \& SCHEERPELTZ, with descriptions of three new species (Coleoptera, Staphylinidae, Aleocharinae). - Beiträge zur Entomologie, Berlin 45 (1): 217-237. - https://www.contributionsto-entomology.org/article/view/1409/1408.

Assing, V. 1997a: A revision of the Western Palaearctic species of Myrmecopora SAULCY, 1864 sensu lato and Eccoptoglossa Luze, 1904 (Coleoptera, Staphylinidae, Aleocharinae, Falagriini). - Beiträge zur Entomologie, Berlin 47 (1): 69-151. - https://www.contributionsto-entomology.org/article/view/1446/1445.

Assing, V. 1997b: A revision of Othius Stephens, 1829. III. The species of the Western Palaearctic region exclusive of the Atlantic Islands (Coleoptera, Staphylinidae: Xantholininae). - Nova Supplementa Entomologica, Berlin 10: 3-130.

Assing, V. 1999: A revision of the species of Geostiba Thomson 1858 from Greece and Cyprus (Coleoptera, Staphylinidae, Aleocharinae). - Linzer Biologische Beiträge 31 (2): 845-928. - https://www.zobodat.at/ pdf/LBB_0031_2_0845-0928.pdf. 
Assing, V. 2000: A revision of the species of Geostiba Thomson 1858 and Paraleptusa Peyerimhoff 1901 of Greece: Supplement I, including some species from Albania, Macedonia, Bulgaria, and Turkey (Coleoptera: Staphylinidae, Aleocharinae). - Linzer Biologische Beiträge 32 (2): 1007-1031. - https:// www.zobodat.at/pdf/LBB_0032_2_1007-1031.pdf.

Assing, V. 2001: A revision of the species of Geostiba Thоmson of the Balkans and Turkey. V. New species, a new synonym, new combinations, and additional records (Coleoptera: Staphylinidae, Aleocharinae). - Linzer Biologische Beiträge 33 (2): 689-707. - https://www.zobodat.at/pdf/LBB_0033_2_06890707.pdf.

Assing, V. 2002: New species of Staphylinidae from Greece (Insecta: Coleoptera). - Reichenbachia 34 (33): 277-284.

Assing, V.2003a: The first species of the subgenus Eurysunius Reitter, genus Astenus Dejean, from Crete (Insecta: Coleoptera: Staphylinidae: Paederinae). Entomologische Abhandlungen 60: 121-123.

Assing, V. 2003b: A revision of Othiini. XIII. Horizontal and vertical distribution of Othius, new species, and additional records (Coleoptera: Staphylinidae: Staphylininae). - Entomological Problems 33 (1-2): 69-88.

Assing, V. 2003c: On the taxonomy of Gyrohypnus LEACH: new synonymies, new species, and a key to the Western Palaearctic and Middle Asian representatives of the genus (Insecta: Coleoptera: Staphylinidae). - Entomologische Blätter 99 (1-3): 55-81.

Assing, V. 2004a: A revision of the Medon species of the Eastern Mediterranean and adjacent regions (Insecta: Coleoptera: Staphylinidae: Paederinae). - Bonner Zoologische Beiträge 52 (1-2): 33-82. - https:// www.zobodat.at/pdf/Bonner-Zoologische-Beitraege_52_0033-0082.pdf.

Assing, V. 2004b: New species and records of Staphylinidae from Greece (Insecta: Coleoptera). - Linzer Biologische Beiträge 36 (2): 593-613. - https://www. zobodat.at/pdf/LBB_0036_2_0593-0613.pdf.

Assing, V. 2004c: New species and records of Staphylinidae from Turkey III (Insecta: Coleoptera). - Linzer Biologische Beiträge 36 (2): 669-733. - https://www. zobodat.at/pdf/LBB_0036_2_0669-0733.pdf.

Assing, V.2005a: A revision of the genus Leptobium CASEY (Coleoptera: Staphylinidae: Paederinae). - Stuttgarter Beiträge zur Naturkunde, Serie A (Biologie), Nr. 673: 1-182. - https://www.zobodat.at/pdf/Stuttgarter-Beitraege-Naturkunde_673_A_0001-0182.pdf.

Assing, V. 2005b: On the Western Palaearctic species of Drusilla LeAch, with special reference to the species of the eastern Mediterranean (Coleoptera: Staphylinidae, Aleocharinae). - Koleopterologische Rundschau 75: 111-149. - https://www.zobodat.at/ pdf/KOR_75_2005_0111-0149.pdf.
Assing, V. 2005c: Two new species and new records of Staphylinidae from the Greek island Lesbos (Insecta: Coleoptera). - Linzer Biologische Beiträge 37 (2): 1035-1046. - https://www.zobodat.at/pdf/ LBB_0037_2_1035-1046.pdf.

Assing, V. 2006: New species and records of Staphylinidae from Greece, with two new synonymies (Insecta: Coleoptera). - Linzer Biologische Beiträge 38 (1): 333-379. - https://www.zobodat.at/pdf/LBB_0038_ 1_0333-0379.pdf.

Assing, V. 2007a: On the Xantholinini of Turkey and adjacent regions (Coleoptera: Staphylinidae: Staphylininae). - Zootaxa 1474: 1-54. - https://www.mapress. com/j/zt/article/view/zootaxa.1474.1.1/26831.

Assing, V. 2007b: A revision of Palaearctic Lobrathium Mulsant \& Rey. III. New species, new synonyms, and additional records (Coleoptera: Staphylinidae, Paederinae). - Linzer Biologische Beiträge 39 (2): 731-755. - https://www.zobodat.at/ pdf/LBB_0039_2_0731-0755.pdf.

Assing, V. 2007c: A revision of the species of Pronomaea ERICHSON of the Western Palaearctic region, including Middle Asia (Coleoptera: Staphylinidae: Aleocharinae: Pronomaeini). - Beiträge zur Entomologie, Keltern 57 (2): 367-396. - https://www.contributions-to-entomology.org/article/view/1710/1709.

Assing, V. 2007d: Four new species and additional records of Geostiba from Turkey and Crete, and a new synonymy (Coleoptera: Staphylinidae, Aleocharinae). - Linzer Biologische Beiträge 39 (2): 777-790. - https://www.zobodat.at/pdf/LBB_0039_2_07770790.pdf.

Assing, V. 2007e: A revision of Western Palaearctic Medon. V. A new species from Iran and additional records (Coleoptera: Staphylinidae, Paederinae). Linzer Biologische Beiträge 39 (1): 23-32. - https:// www.zobodat.at/pdf/LBB_0039_1_0023-0032.pdf.

Assing, V. 2007f: Two new species and additional records of "small"-eyed Quedius from the Eastern Mediterranean (Coleoptera: Staphylinidae: Staphylininae). - Beiträge zur Entomologie, Keltern 57 (2): 335-345. - https:/www.contributions-to-entomology.org/ article/view/1707/1706.

Assing, V. 2008a: A revision of the Micrillus species of the Palaearctic region, with notes on two species from adjacent parts of the Afrotropical and Oriental regions (Coleoptera: Staphylinidae: Paederinae). - Stuttgarter Beiträge zur Naturkunde Serie A, Neue Serie 1: 301-344. - https://www.zobodat.at/pdf/StuttgarterBeitraege-Naturkunde_NS_1_A_0301-0344.pdf.

Assing, V. 2008b: A revision of the Habrocerinae of the world. IV. A new species of Habrocerus from China and additional records (Coleoptera: Staphylinidae). - Beiträge zur Entomologie, Keltern 58 (1): 135-144. - https://www.contributions-to-entomology.org/article/view/1722/1721. 
Assing, V. 2008c: A revision of the Sunius species of the Western Palaearctic region and Middle Asia (Coleoptera: Staphylinidae: Paederinae). - Linzer Biologische Beiträge 40 (1): 5-135. - https://www.zobodat.at/pdf/ LBB_0040_1_0005-0135.pdf.

Assing, V. 2008d: On the taxonomy and zoogeography of some Palaearctic Paederinae and Xantholinini (Coleoptera: Staphylinidae). - Linzer Biologische Beiträge 40 (2): 1237-1294. - https://www.zobodat.at/pdf/ LBB_0040_2_1237-1294.pdf.

Assing, V. 2008e: Four new species and additional records of Palaearctic Sunius, with two new synonymies (Coleoptera: Staphylinidae: Paederinae). - Beiträge zur Entomologie, Keltern 58 (2): 455-470. - $\quad$ https://www.contributions-to-entomology.org/ article/view/1735/1734.

Assing, V. 2008f: A revision of Othiini. XVI. Four new species of Othius from the Himalaya and China, and additional records (Coleoptera: Staphylinidae, Staphylininae). - Koleopterologische Rundschau 78: 245-263. - https://www.zobodat.at/pdf/KOR_ 78_2008_0245-0263.pdf.

Assing, V. 2009a: A revision of Leptobium CASEY. IV. Three new species and additional records (Coleoptera: Staphylinidae: Paederinae). - Stuttgarter Beiträge zur Naturkunde Serie A, Neue Serie 2: 227-236. - https:// www.zobodat.at/pdf/Stuttgarter-Beitraege-Naturkunde_NS_2_A_0227-0236.pdf.

Assing, V. 2009b: A revision of Leptobium CASEy. III. A new species from Iran and additional records (Coleoptera: Staphylinidae: Paederinae). - Linzer Biologische Beiträge 41 (2): 1247-1252. - https:// www.zobodat.at/pdf/LBB_0041_2_1247-1252.pdf.

Assing, V. 2009c: On the Western Palaearctic and Middle Asian species of Ochthephilum STEPHENS, with notes on Cryptobium koltzei Eppelsheim (Coleoptera: Staphylinidae: Paederinae: Cryptobiina). - Linzer Biologische Beiträge 41 (1): 397-426. - https://www. zobodat.at/pdf/LBB_0041_1_0397-0426.pdf.

Assing, V. 2009d: On the Pseudomedon species of the Palaearctic region (Coleoptera: Staphylinidae: Paederinae). - Linzer Biologische Beiträge 41 (2): 1175-1189. - https://www.zobodat.at/pdf/LBB_ 0041_2_1175-1189.pdf.

Assing, V. 2010a: A revision of the genus Luzea (Coleoptera: Staphylinidae: Paederinae). - Deutsche Entomologische Zeitschrift 57 (1): 117-135.

Assing, V. 2010b: A revision of Achenium (Coleoptera: Staphylinidae: Paederinae). - Nova Supplementa Entomologica 21: 1-190.

Assing, V. 2010c: A revision of Othiini. XVII. A new species from China and additional records (Coleoptera: Staphylinidae: Staphylininae). - Linzer Biologische Beiträge 42 (2): 1077-1091. - https:// www.zobodat.at/pdf/LBB_0042_2_1077-1091.pdf.
Assing, V. 2012: A revision of East Palaearctic Lobrathium (Coleoptera: Staphylinidae: Paederinae). - Bonn Zoological Bulletin 61 (1): 49-128. - https://www.google.com/url?sa=t\&rct=j\&q=\&esr $\mathrm{c}=\mathrm{s} \&$ source $=$ web \&cd $=1$ \&ved $=2 \mathrm{ahUKEwiN} 1 \mathrm{qGo}-$ LjAhVBoVwKHfn1D8EQFjAAegQIAhAC\&url=htt ps\%3A\%2F\%2Fwww.zoologicalbulletin.de\%2FBzB_ Volumes\%2FVolume_61_1\%2F049_128_ BzB61_1_Assing_Vpdf\&usg=AOvVaw0Yn2AsjWEjzc5wOF0i8xA.

Assing V. 2013a: On the Staphylinidae (Coleoptera) of Crete, Greece. - Stuttgarter Beiträge zur Naturkunde A, Neue Serie 6: 83-102. - https://www. zobodat.at/pdf/Stuttgarter-Beitraege-Naturkunde_ NS_6_A_0083-0102.pdf.

Assing, V. 2013b: A revision of Othiini XVIII. Two new species from China and additional records (Coleoptera: Staphylinidae: Staphylininae). - Koleopterologische Rundschau 83: 73-92. - https://www. zobodat.at/pdf/KOR_83_2013_0073-0092.pdf.

Assing, V. 2013c: A revision of Palaearctic Medon IX. New species, new synonymies, a new combination, and additional records (Coleoptera: Staphylinidae: Paederinae). - Entomologische Blätter und Coleoptera 109: 233-270.

Assing, V. 2013d: On the Staphylinidae of Rhodes, Greece (Insecta: Coleoptera). - Linzer Biologische Beiträge 45 (2): 1587-1613. - https://www.zobodat. at/pdf/LBB_0045_2_1587-1613.pdf.

Assing, V. 2014a: A revision of Palaearctic Lobrathium. V. Three new species from the Himalaya and China, a new synonymy, and additional records (Coleoptera: Staphylinidae: Paederinae). - Linzer Biologische Beiträge 45 (1): 429-441. - https://www.zobodat.at/ pdf/LBB_0046_1_0429-0441.pdf.

Assing, V. 2014b: On the Bolitochara species of the West Palaearctic region (Coleoptera: Staphylinidae: Aleocharinae). - Stuttgarter Beiträge zur Naturkunde A, Neue Serie 7: 33-63. - https://www.zobodat.at/pdf/ Stuttgarter-Beitraege-Naturkunde_NS_7_A_00330063.pdf.

Assing, V. 2014c: On the Staphylinidae of Sardinia, Italy (Insecta: Coleoptera). - Linzer Biologische Beiträge 45 (1): 415-428. - https://www.zobodat.at/pdf/ LBB_0046_1_0415-0428.pdf.

Assing, V. 2015a: On the Staphylinidae (Coleoptera) of Crete II. Seven new species, a new synonymy, and additional records. - Stuttgarter Beiträge zur Naturkunde A, Neue Serie 8: 95-112. - https://www. zobodat.at/pdf/Stuttgarter-Beitraege-Naturkunde_ NS_8_A_0095-0112.pdf.

Assing, V. 2015b: A revision of Sunius XV. Six new species from Kyrgyzstan and the Himalaya, and additional records (Coleoptera: Staphylinidae: Paederinae). - Contributions to Entomology 65 (2): 287-295. https://www.contributions-to-entomology.org/article/ view/1884/1883. 
Assing, V. 2015c: On the Staphylinidae of the Greek island Samos (Coleoptera: Staphylinidae). - Koleopterologische Rundschau 85: 81-102. - https://www. zobodat.at/pdf/KOR_85_2015_0081-0102.pdf.

Assing, V. 2015d: On the Staphylinidae of the Greek island Chios (Insecta: Coleoptera). - Linzer Biologische Beiträge 47 (1): 43-55. - https://www.zobodat. at/pdf/LBB_0047_1_0043-0055.pdf.

Assing, V. 2016a: On the Staphylinidae of the Greek island Karpathos (Insecta: Coleoptera). - Linzer Biologische Beiträge 48 (1): 235-263. - https://www. zobodat.at/pdf/LBB_0048_1_0235-0263.pdf.

Assing, V. 2016b: On the Staphylinidae of the Greek island Lesbos II, with supplementary notes on the fauna of Samos and Chios (Coleoptera: Staphylinidae). - Koleopterologische Rundschau 86: 103-138. - https://www.zobodat.at/pdf/KOR_86_2016_01030138.pdf.

Assing, V. 2017a: A revision of Leptobium CAsey. VIII. A new species from Iran, a new synonymy, and additional records (Coleoptera: Staphylinidae: Paederinae). - Linzer Biologische Beiträge 49 (1): 275-284. - https://www.zobodat.at/pdf/LBB_0049_1_02750284.pdf.

Assing, V. 2017b: Taxonomic and faunistic notes on some West Palaearctic and Middle Asian Xantholinini, with a revalidation and new synonymies (Coleoptera: Staphylinidae: Staphylininae). - Linzer Biologische Beiträge 49 (1): 235-252. - https://www.zobodat.at/ pdf/LBB_0049_1_0235-0252.pdf.

Assing, V. 2017c: On the Staphylinidae of the Greek island Ikaría, with supplementary notes on the fauna of Samos (Coleoptera: Staphylinidae). - Koleopterologische Rundschau 87: 89-116. - https://www. zobodat.at/pdf/KOR_87_2017_0089-0116.pdf.

Assing, V. 2017d: On the Staphylinidae of the Greek island Kos, with an appendix on Carabidae and additional records from other islands (Insecta: Coleoptera). - Linzer Biologische Beiträge 49 (1): 191-205. - https://www.zobodat.at/pdf/LBB_0049_1_01910205.pdf.

Assing, V. 2018a: Revision of the Cousya species of the West Palaearctic Region (Coleoptera: Staphylinidae: Aleocharinae). - Integrative Systematics 1: 79-127. https://bioone.org/journalArticle/Download?fullDO I=10.18476\%2Finsy.v01.a9.

Assing, V. 2018b: A revision of Medon. XI. Five new species, additional records, and the first confirmed records from the Oriental region (Coleoptera: Staphylinidae: Paederinae). - Contributions to Entomology 68 (1): 69-81. - https://www.contributions-to-entomology.org/article/view/1961/1979.

Assing, V. 2018c: On the Staphylinidae of Crete III. The first records of endogean fauna (Coleoptera: Staphylinidae: Leptotyphlinae, Aleocharinae). - Linzer Biologische Beiträge 50 (1): 7-15. - https://www. zobodat.at/pdf/LBB_0050_1_0007-0015.pdf.
Assing, V. 2018d: On some Myllaena species in the East Mediterranean and Caucasus regions (Coleoptera: Staphylinidae: Aleocharinae). - Linzer Biologische Beiträge 50 (2): 1015-1032. - https://www.zobodat. at/pdf/LBB_0050_2_1015-1032.pdf.

Assing, V. 2019a: Revision of the Baeoglena species of the West Palaearctic Region (Coleoptera: Staphylinidae: Aleocharinae). - Contributions to Entomology 69 (1): 1-32. - https://www.contributions-to-entomology.org/article/view/1990/2001.

Assing, V. 2019b: Three new species and additional records of Oedichirus (Coleoptera: Staphylinidae: Aleocharinae). - Linzer Biologische Beiträge 51 (1): 33-42. - https://www.zobodat.at/pdf/LBB 0051_1_0033-0042.pdf.

Assing, V. \& Feldmann, B. 2012: On the Staphylinidae of Israel (Insecta: Coleoptera). - Linzer Biologische Beiträge 44 (1): 351-363. - https://www.zobodat.at/ pdf/LBB_0051_1_0033-0042.pdf.

Assing, V.; Schülke, M.; Brachat, V. \& Меybohm, H. 2018: On the Staphylinidae of the Greek island Corfu (Insecta: Coleoptera). - Contributions to Entomology 68 (1): 31-67. - https://www.contributions-to-entomology.org/article/view/1958/1975.

Assing, V.; Brachat, V. \& Мeybohm, H. 2019: Monograph of the Staphylinidae of Crete (Greece). Part II. Descriptions of new species (Insecta: Coleoptera). Contributions to Entomology 69 (2): 239-289.

Assing, V. \& Vogel, J. 2019: The mother of synonyms: on the Meotica species of the Palaearctic region (Coleoptera: Staphylinidae: Aleocharinae: Oxypodini). - Linzer Biologische Beiträge 51 (2) (in press).

Assing, V. \& Wunderle, P. 1995: A revision of the species of the subfamily Habrocerinae (Coleoptera: Staphylinidae) of the world. - Revue Suisse de Zoologie 102 (2): 307-359.

Assing, V. \& Wunderle, P. 2008: On the Alevonota species of the Western Palaearctic region (Coleoptera: Staphylinidae: Aleocharinae: Athetini). - Beiträge zur Entomologie, Keltern 58 (1): 145-189. - https:// www.contributions-to-entomology.org/article/ view/1723/1722.

Bernhauer, M. 1905: 13. Folge neuer Staphyliniden der paläarktischen Fauna, nebst Bemerkungen. - Verhandlungen der Kaiserlich-Königlichen Zoologisch-Botanischen Gesellschaft in Wien 55: 580-596. - https://www.zobodat.at/pdf/VZBG_55_0580-0596. pdf.

Bernhauer, M. 1908: 14. Folge neuer Staphyliniden der paläarktischen Fauna, nebst Bemerkungen. - Verhandlungen der Kaiserlich-Königlichen Zoologisch-Botanischen Gesellschaft in Wien 58: 32-41. - https://www.zobodat.at/pdf/VZBG_58_0032-0041. pdf.

Bernhauer, M. 1915: Neue Staphyliniden des paläarktischen Faunengebietes. - Wiener Entomologische Zeitung 34 (5-7): 69-81. - https://www.zobodat.at/ pdf/WEZ_34_0069-0081.pdf. 
Bernhauer, M. 1935: Neuheiten der paläarktischen Staphylindenfauna. - Koleopterologische Rundschau 21: 39-48. - https://www.zobodat.at/pdf/KOR_ 21_1935_0039-0048.pdf.

Bernhauer, M. 1939: Übersicht der tetracarinatus-Gruppe der Gattung Oxytelus Grav., Untergattung Anotylus Tномs., mit den Beschreibungen zweier neuer Arten. - Koleopterologische Rundschau 25: 70-75. - https:// www.zobodat.at/pdf/KOR_25_1939_0070-0075.pdf.

Besuchet, C. 1955: Monographie des Bibloplectus et Pseudoplectus paléarctiques (Col. Pselaphidae). Mitteilungen der Schweizerischen Entomologischen Gesellschaft 28 (2): 153-209. - https://www.e-periodica.ch/cntmng?pid=seg-001:1955:28::579.

Blattný, W. \& Blattný, C. 1916: Die von PaganettiHummler im Jahre 1914 auf einer Forschungsreise in Kreta gesammelten Pselaphiden und Scydmaeniden. - Zeitschrift für Wissenschaftliche Insektenbiologie. Beilage Neue Beiträge zur Systematischen Insektenkunde 1: 1-6.

Bordoni, A. 1975: Xantholinini raccolti da Marcello Cerruti in Grecia e nelle isole dell'Egeo e descrizione dello Xantholinus (Calolinus) pantokratoris n. sp. di Corfu (Coleoptera Staphylinidae). - Fragmenta Entomologica 11: 139-147.

Bordoni, A. 1976: Studi sulla sistematica e la geonemia degli Xantholinus. IX. Xantholinus del Museo di Storia Naturale di Ginevra raccolti nel bacino del Mediterraneo ed appunti su alcuni altri Xantholinini. Prima nota (Col. Staphylinidae). - Revue Suisse de Zoologie 83 (1): 83-103.

Bordoni, A. 1977: Studi sui Paederinae. I. Appunti sui Domene Fauvel della Penisola Iberica e descrizione di due nuove specie mediterranee (Col. Staphylinidae). - Nouvelle Revue d'Entomologie 7 (2): 145-151.

Bordoni, A. 1984: Su alcuni Leptobium CASEy del Mediterraneo (Col. Staphylinidae). - Bollettino della Società Entomologica Italiana 116: 83-88.

Bordoni, A. 1986: Xantholiniinae e Paederinae del Museo di Storia Naturale di Prag e descrizione di nuovi Lathrobiini (Col. Staphylinidae). - Frustula Entomologica (N.S.) 7/8: 385-394.

Bordoni, A. 2007: Palaearctic Xantholinini of the Museum of Zoology in Lund, Sweden, with description of two new species (Coleoptera, Staphylinidae). $182^{\circ}$ contribution to the knowledge of the Staphylinidae. - Zootaxa 1624: 59-68. - https://www.mapress. $\mathrm{com} / \mathrm{j} / \mathrm{zt} /$ article/view/zootaxa.1624.1.6/28298.

Bordoni, A. 2009: Palaearctic Lathrobiina of the Museum of Zoology of Lund with description of Lobrathium candicum n. sp. from Crete (Coleoptera Staphylindae) [sic]. - Bollettino della Società Entomologica Italiana 141 (3): 139-142.

Bordoni, A. 2014: New data on the Palaearctic Xantholinini. $8^{\circ}$. New records and illustrations on some species (Coleoptera: Staphylinidae). - Onychium 10: 56-67. - http://www.onychium.it/sites/default/files/ articoli/Bordoni_2014b_onychium10_0.pdf.
Bordoni, A. 2016: New data on the Palaearctic Xantholinini. $9^{\circ}$. Three new Mediterranean species of Xantholinus (Coleoptera: Staphylinidae). - Fragmenta Entomologica 48 (1): 25-28.

Bordoni, A. 2017: New data on the Palaearctic Xantholinini. 12. New species, new designations and new records (Coleoptera: Staphylinidae). - Onychium 13: 107-115. - http://www.onychium.it/sites/default/ files/articoli/Bordoni_2017a_Onychium13.pdf.

Brundin, L. 1944: Monographie der palaearktischen Arten der Atheta-Untergattung Hygroecia (Coleoptera, Staphylinidae). - Annalen des Naturhistorischen Museums in Wien 53 [1942]: 129-301, pl. 13-40. - https://www.zobodat.at/pdf/ANNA_53_2_01290301.pdf.

Castellini, G. 1996: Revisione del genere Leptomastax Pirazzolli, 1855 (Coleoptera, Scydmaenidae). - Atti del Museo Civico di Storia Naturale di Grosseto 15 (Suppl.) [1994]: 1-137.

Cellinese, N.; Smith, S. A.; Edwards, E. J.; Sang-Tae, K.; Haberle, R. C.; Avramakis, M. \& Donoghue, M. J. 2009: Historical biogeography of the endemic Campanulaceae of Crete. - Journal of Biogeography 36: 1253-1269. - https://onlinelibrary.wiley.com/doi/ epdf/10.1111/j.1365-2699.2008.02077.x.

Ciceroni, A.; Puthz, V. \& Zanetti, A. 1995: Coleoptera Staphylinidae. Fasciculo 48. - In: Minelli, A.; Ruffo, S. \& LA Posta, S. (eds), Checklist delle specie della fauna italiana. - Edizioni Calderini, Bologna: $65 \mathrm{pp}$.

Coiffait, H. 1970: Formes nouvelles ou mal connues des genres Medon et Hypomedon. - Annales des Spéléologie 24 [1069]: 701-727.

Coiffait, H. 1972: Nouveaux Staphylinidae euporéens [sic]. - Nouvelle Revue d'Entomologie 2: 79-83.

Coiffait, H. 1973: Staphylinides nouveaux ou mal connus d'Europe, d'Afrique du Nord et du MoyenOrient. Les sous-genres du genre Bledius. - Nouvelle Revue d'Entomologie 3 (2): 107-123.

Coiffait, H. 1976: Staphylinidae récoltés par M. Cerruti dans la région méditerranéenne orientale. - Fragmenta Entomologica 12: 81-101.

Coiffait, H. 1980: Staphylinides nouveaux. - Nouvelle Revue d'Entomologie 10: 37-49.

Cuccodoro, G. 2017: Review of the observations of aggregates of Steninae reported since 1856 (Coleoptera Staphylinidae). - Biodiversity Journal 8 (1): 123-144. - http://www.biodiversityjournal.com/ pdf/8(1)_123-144.pdf.

FAgel, G. 1951: Contribution a la connaissance des Staphylinidae. VIII. - Note sur certains Ancyrophorus européens. - Bulletin Institut Royal des Sciences Naturelles de Belgique 27 (46): 1-7.

FranZ, H. 1971: Untersuchungen über die paläarktischen Arten der Gattungen Euthia STEPH. und Veraphis CASEy (Col. Scydmaenidae). - Eos, Revista Española de Entomología 46: 57-84. 
Frisch, J. 1994: Neue Arten der Gattung Scopaeus ERICHSON aus Griechenland, Anatolien und dem Iran (Coleoptera, Staphylinidae, Paederinae). - Coleoptera Schwanfelder Coleopterologische Mitteilungen 2: $1-46$.

Frisch, J. 1997: Zur Synonymie des Scopaeus (Microscopaeus) pilicornis BAUd, 1869 (Coleoptera, Staphylinidae: Paederinae), einer verkannten Art des östlichen Mediterraneums. - Mitteilungen des Zoologischen Museums Berlin 73 (1): 95-101.

Frisch, J. 1998: A revision of some West Palaearctic species of Scopaeus Erichson (Coleoptera, Staphylinidae, Paederinae). - Revue Suisse de Zoologie 105 (1): 89-124.

FrISCH, J. 1999a: A revision of Scopaeus debilis group, with description of a new species frrom Madagascar (Coleoptera, Staphylinidae, Paederinae). - Revue Suisse de Zoologie 106 (2): 361-383.

Frisch, J. 1999b: Scopaeus korelli n. sp. from North Anatolia (Coleoptera, Staphylinidae), with synonymical and distributional notes on the Scopaeus elegans species group. - Entomologische Blätter 95 (2-3): $159-165$.

Frisch, J. 2010: On the taxonomy and biogeography of West Palaearctic Scopaeina Mulsant \& Rey (Staphylinidae, Paederinae). - Deutsche Entomologische Zeitschrift 57 (2): 159-202. - https://onlinelibrary. wiley.com/doi/epdf/10.1002/mmnd.201000016.

GiLdenkov, M. Y. 2015: Fauna Carpelimus starogo sveta (Coleoptera: Staphylinidae). - Izdatelstvo SmolGU, Smolensk: 1-413.

Hadjisterkotis, E. 2012: Chapter 2. The arrival of elephants on the island of Cyprus and their subsequent accumulation in fossil sites. - In: Aranovich, M. \& Dufresne, O. (eds), Elephants: ecology, behavior and conservation. - Nova Science Publishers, New York: 202 pp.

Heyden, L. v. 1884: Coleopterologische Ausbeute des Baron H. v. Maltzan von der Insel Creta. - Deutsche Entomologische Zeitschrift 28 (2): 363-368.

Horion, A. 1963: Faunistik der mitteleuropäischen Käfer. Band IX: Staphylinidae. 1. Teil. Micropeplinae bis Euaesthetinae. - A. Feyel, Überlingen - Bodensee: $412 \mathrm{pp}$.

Horion, A. 1965: Faunistik der mitteleuropäischen Käfer. Band X: Staphylinidae. 2. Teil. Paederinae bis Staphylininae. - Ph. C. W. Schmidt, Überlingen - Bodensee: $335 \mathrm{pp}$.

Horion, A. 1967: Faunistik der mitteleuropäischen Käfer. Band XI: Staphylinidae. 3. Teil. Habrocerinae bis Aleocharinae. - Ph. C. W. Schmidt, Überlingen Bodensee: 419 pp.

Horn, W.; Kahle, I.; Friese, G. \& Gaedike, R. (1990): Collectiones entomologicae. Ein Kompendium über den Verbleib entomologischer Sammlungen der Welt bis 1960. - Akademie der Landwirtschaftswissenschaften der Deutschen Demokratischen Republik, Berlin: 573 pp.
Jászay, T. \& HLaváč, P. 2006: A revision of the Palaearctic species of the genus Dropephylla (Coleoptera: Staphylinidae: Omaliinae). - Entomological Problems 36 (1): 31-62.

Jolivet, L. \& Brun, J.-P. 2010: Cenozoic geodynamic evoloution of the Aegean. - Journal of Earth Science 99: 109-138.

Karaman, Z. 1955: Revision des Tribus Tychini (Col. Psel.) mit besonderer Berücksichtigung der balkanischen Arten. - Acta Musei Macedonici Scientiarum Naturalium 3: 105-144.

Ketmaier, V.; Giusti, F. \& Caccone, A. 2006: Molecular phylogeny and historical biogeography of the land snail genus Solatopupa (Pulmonata) in the periTyrrhenian area. - Molecular Phylogenetics and Evolution 39: 439-451. - https://doi.org/10.1016/j. ympev.2005.12.008.

KocH, C. 1936: Wissenschaftliche Ergebnisse der entomologischen Expeditionen Seiner Durchlaucht des Fürsten Alessandro C. della Torre e Tasso nach Aegypten und auf die Halbinsel Sinai. XIII. Staphylinidae. - Pubblicazini del Museo Entomologico "Pietro Rossi", Duino 1: 115-232.

KraAtZ, G. 1857: Naturgeschichte der Insecten Deutschlands. Erste Abtheilung Coleoptera. Zweiter Band. Lieferung 3-6. - Nicolai, Berlin: 377-1080.

KraAtZ, G. 1858a: Beitrag zur Käferfauna Griechenlands. Zweites Stück: Palpicornia, Silphales, Scydmaenidae, Pselaphidae, Staphylinidae. - Berliner Entomologische Zeitschrift 2: 37-67. - https://www.zobodat.at/ pdf/Deutsche-Ent-Zeitschrift_2_0037-0067.pdf.

KrAATZ, G. 1858b: Beitrag zur Käferfauna Griechenlands. Drittes Stück: Staphylinidae (Schluss), Trichopterygia, Histeridae, Phalacridae, Nitidulariae, Trogositarii, Colydii, Cucujidae, Cryptophagidae, Thorictidae, Mycetophagidae, Dermestini, Byrrhii. - Berliner Entomologische Zeitschrift 2: 123-148. - https://www.zobodat.at/pdf/Deutsche-Ent-Zeitschrift_2_0123-0148.pdf.

LöвL, I. 1998: On new and old replacement names in Palaearctic Pselaphinae (Coleoptera: Staphylinidae). - Mitteilungen der Schweizerischen Entomologischen Gesellschaft 71: 463-465.

Lohse, G. A. \& Steel, W. 1961: New species of Lesteva LATREILle from the eastern Mediterranean (Coleoptera: Staphylinidae). - Proceedings of the Royal Entomological Society of London (B) 30: 72-76.

LoKAY, E. 1913: Nové staphylinidy palaearktičté (Coleoptera: Staphylinidae). - Časopis Československé Společnosti Entomologické 10: 136-140.

LoKAY, E. 1921: Noví staphylinidi palaearktičtí (Coleoptera: Staphylinidae). - Časopis Československé Společnosti Entomologické 18 (1-2): 71-72.

Makranczy, Gy. 2014: Revision of the genus Ochthephilus Mulsant \& Rey, 1856 (Coleoptera: Staphylinidae, Oxytelinae). - Revue Suisse de Zoologie 121 (4): 457-694. 
MAřAn, J. 1935: Nové a zajímavé formy rodu Quedius STEPH. z ostrova Kréty. De novis et raris formis generis Quedius STPEPH. ex insula Creta. - Časopis Československé Společnosti Entomologické 32: 8-9.

Мечвонм, H. 2008: Scydmoraphes-Arten von Kreta, Karpathos und Rhodos sowie des benachbarten türkischen Festlandes (Coleoptera, Scydmaenidae). Entomologische Blätter 103/104: 3-9.

Меүвонм, H. 2009: Die Gattung Leptocharis und der Fundort Crête Magri (Coleoptera: Scydmaenidae). Linzer Biologische Beiträge 41 (1): 737-742. - https:// www.zobodat.at/pdf/LBB_0041_1_0737-0742.pdf.

Oertzen, E. v. 1887: Verzeichniss der Coleopteren Griechenlands und Cretas, nebst einigen Bemerkungen über ihre geographische Verbreitung und 4 die Zeit des Vorkommens einiger Arten betreffenden Sammelberichten. - Berliner Entomologische Zeitschrift 30 [1886] (2): 189-293.

PACE, R. 1996: Descrizione di nuove specie e sottospecie del genere Geostiba (Coleoptera, Staphylinidae). Bollettino dell'Associazione Romana di Entomologia 50 (1995): 7-43.

PACE, R. 2002: Aloconota minoica n. sp., eine neue Art von der Insel Kreta (Griechenland) (Coleoptera, Staphylinidae). - Veröffentlichungen Naturkundemuseum Erfurt 21: 205-208.

Perissoratis, C. \& Conispoliatis, N. 2003: The impacts of sea-level changes during latest Pleistocene and Holocene times on the morphology of the Ionian and Aegean seas (SE Alpine Europe). - Marine Geology 196: $145-156$.

Pic, M. 1903: Deux captures intéressantes. Diagnoses de divers coléoptères. - L'Échange, Revue Linnéenne 19 (224): 145-147.

Puthz, V. 1968: Die Stenus- und Megalopinus-Arten Motschulskys und Bemerkungen über das Subgenus Tesnus Rey, mit einer Tabelle der paläarktischen Vertreter (Coleoptera, Staphylinidae). - Notulae Entomologicae 48: 197-219.

Puthz, V. 1971: Neue Stenus-Arten der erichsoni-ludyi-coarcticollis-Gruppe (Coleoptera, Staphylinidae). - Bulletin de l'Institut Royal des Sciences Naturelles de Belgique 47 (14): 1-9.

Puthz, V. 1972a: Zur Staphylinidenfauna des Balkans: Die bisher aus Jugoslawien und angrenzenden Ländern bekannten Steninen (Coleoptera, Staphylinidae). - Wissenschaftliche Mitteilungen des Bosnisch-Herzegovinischen Landesmuseums (C Naturwissenschaft) [1971] 1: 239-292.

Puthz, V. 1972b: Zur Staphylinidenfauna des Balkans: Die bisher aus Jugoslawien und angrenzenden Ländern bekannten Steninen (Coleoptera, Staphylinidae). 1. Nachtrag. - Wissenschaftliche Mitteilungen des Bosnisch-Herzegovinischen Landesmuseums (C - Naturwissenschaft) 2: 167-179.

Puthz, V. 1977: Neue westpaläarktische Stenus - Arten aus dem Genfer Museum (Coleoptera, Staphylinidae). - Revue Suisse de Zoologie 84 (1): 109-117.
Puthz, V. 1979: Lesteva-Funde aus Griechenland (Staphylinidae). - Entomologische Blätter 74 (3): 190.

Puthz, V. 1980: 1984 Faunistische Notizen aus Hessen (Hist., Liod., Scydm., Staph., Canth., Elat., Throsc.). Entomologische Blätter 75 (3): 172-173.

Puthz, V. 2008: Stenus Latreille und die segenreiche Himmelstochter (Coleoptera, Staphylinidae). - Linzer Biologische Beiträge 40 (1): 137-230. - https://www. zobodat.at/pdf/LBB_0040_1_0137-0230.pdf.

ReitTer, E. 1885: Neue Coleopteren aus Europa und den angrenzenden Ländern, mit Bemerkungen über bekannte Arten. - Deutsche Entomologische Zeitschrift 29 (2): 353-392.

Reitter, E. 1906: Übersicht der mir bekannten, palaearktischen Arten der Coleopteren-Gattung Oedichirus Er. - Wiener Entomologische Zeitung 25 (8-9): 263-264.

SAbella, G. 2002: Description of Tychus lagrecai sp. n. (Coleoptera, Stapghylinidae, Pselaphinae) from Crete island (Greece). - Bollettino dell'Academia Gioenia di Scienze Naturali, Catania 35 (361): 611-616.

SAbella, G.; Brachat, V. \& BüCKLE, C. 1998: Revisione dei Tychus del gruppo dalmatinus, con descrizione di novi taxa (Coleoptera: Pselaphidae). - Annales de la Société Entomologique de France (N. S.) 34 (3): $217-244$

Sabella, G.; Bückle, C.; Brachat, V. \& Besuchet, C. 2004: Revision der paläarktischen Arten der Gattung Brachygluta Thomson, 1859. 1. Teil: Arten mit sekundären männlichen Geschlechtsmerkmalen auf den Abdominaltergiten (Coleoptera, Staphylinidae, Pselaphinae). - Instrumenta Biodiversitatis 6: $1-283$.

SAhlberg, J. R. 1903: Ad cognitionem faunae Coleopterorum Graecae fragmenta. - Öfversigt of Finska Vetenskaps-Societetens Förhandlingar 45 [19021903] (12): 1-9.

Scheerpeltz, O. 1931: Zoologische Forschungsreise nach den Jonischen Inseln und dem Peloponnes von Max Beier. XV. Teil Staphylinidae (Coleoptera). Sitzungsberichte der Österreichischen Akademie der Wissenschaften Mathematisch-Naturwissenschaftliche Klasse, Abteilung I 140 (5-6): 359-460.

Scheerpeltz, O. 1936: Die westpaläarktischen Arten der Gattung Edaphus Leconte (Col. Staphylinidae). - Kolepterologische Rundschau 22 (6): 189-225. - https://www.zobodat.at/pdf/KOR_22_1936_01890225.pdf.

Scheerpeltz, O. 1964: Staphyliniden von der Insel Kreta (Coleoptera). - Annales Historico-Naturales Musei Nationalis Hungarici 56: 297-308.

SchülKe, M. 2009: Zwei neue Arten der Gattung Anotylus Thomson aus der Verwandtschaft von A. complanatus (ERICHson) (Coleoptera, Staphylinidae, Oxytelinae). - Linzer Biologische Beiträge 41 (2): 2009-2024. - https://www.zobodat.at/pdf/ LBB_0041_2_2009-2024.pdf. 
Schülke, M. 2010: Zur Taxonomie und Systematik einiger Arten der Untergattung Bledius LeACH 1819 (Coleoptera, Staphylinidae, Oxytelinae). - Linzer Biologische Beiträge 42 (2): 1495-1509. - https:// www.zobodat.at/pdf/LBB_0042_2_1495-1509.pdf.

Schülke, M. 2012: Zur Verbreitung von Anotylus speculifrons (KraAtz, 1857), A.clypeonitens (Pandellé, 1867) und A. schatzmayri (КосH, 1937) (Coleoptera, Staphylinidae, Oxytelinae). - Entomologische Blätter und Coleoptera 108: 121-130.

Schülke, M. 2019: Zur Identität der von Gaston Fagel beschriebenen Arten der Gattung Mycetoporus MANnerheim, 1830 mit Beschreibung neuer Arten der Mycetoporus baudueri Gruppe (Coleoptera, Staphylinidae, Tachyporinae). - Linzer Biologische Beiträge 51 (1): 473-498. - https://www.zobodat.at/ pdf/LBB_0051_1_0473-0498.pdf.

Schülke, M. \& Kocian, M. 2000: Revision der Artgruppe des Mycetoporus nigricollis Stephens, 1835 (Coleoptera, Staphylinidae, Tachporinae). - Entomologische Blätter 96 (2): 81-126.

Schülke, M. \& Smetana, A. 2015: Staphylinidae, pp. 304-1134. - In: LöBL, I. \& LöBL, D. (eds), Catalogue of Palaearctic Coleoptera. New, updated Edition. Volume 2. Hydrophiloidea - Staphylinoidea. Revised and updated edition. - Leiden: Brill: xxvi + $1702 \mathrm{pp}$.

TóтH, L. 1982: Holyvák II. - Staphylinidae II. VII kötet (Coleoptera II) 6. füzet. 54 ábrával. Magyarország Állatvilága. - Fauna Hungariae 151: 1-110.

Triantis, K. A. \& Mrlonas, M. 2009: Greek Islands, Biology, pp. 388-392. - In: Gillespie R. \& Glague, D. A. (eds), Encyclopedia of Islands. Oakland: University of California Press.

Tronquet, M. 2016: Révison des espèces du genre Hydrosmecta Thomson, 1858 présentes en France continentale, Corse et régions voisines (Coleoptera, Staphylinidae, Aleocharinae). - Revue de l'Association Roussillonnaise d'Entomologie 25 (3): 129-152.
Tronquet, M.; Aberlenc, H.-P.; Allemand, R.; Alonso, C.; Bameul, F.; Barnouin, T.; Berger, P.; Besuchet, C.; Bordat, P.; Boucher, S.; Bouget, C.; Bourdonné, J.-C.; Bouyon, H.; Brustel, H.; Casset, L.; Constantin, R.; Coulon, J.; CoutanCeau, J.-P.; Dauphin, P.; Debreuil, M.; Delobel, A.; Doguet, S.; Duhaldeborde, F.; Gompel, N.; Gomy, Y.; Gourvès, J.; Komeza, N.; Leblanc, P.; Leseigneur, L.; $\quad$ Menet, D.; $\quad$ Moncoutier, B.; Montreuil, O.; Noblecourt, T.; Orousset, J.; Pelletier, J.; Perreau, M.; Perez, C.; Peslier, S.; Petitprêtre, J.; Ponel, P.; Pupier, R.; Queinnec, É.; Queney, P.; Richoux, P.; Rose, O.; Rouault, É.; Schott, L.; SecQ, M.; Soldati, F.; Soldati, L.; Sörensson, M.; Tamisier, J.-P.; Tiberghien, G.; Vincent, R. 2014: Catalogue des Coléoptères de France. - Association Roussillonnaise d'Entomologie, Perpignan: $1052 \mathrm{pp}$.

Zanetti, A. 2011: Contribution to the knowledge of Staphylinidae from southern Sardinia (Coleoptera). Conservazione Habitat Invertebrati 5: 331-352.

Zerche, L. 1988: Zur Taxonomie der Gattung Pseudopsis Newman, 1834 (Insecta, Coleoptera, Staphylinidae, Pseudopsinae). - Reichenbachia 25 (30): 151-155.

Zerche, L. 1990: Monographie der paläarktischen Coryphiini (Coleoptera, Staphylinidae, Omaliinae). Berlin: Akademie der Landwirtschaftswissenschaften der Deutschen Demokratischen Republik: 1-413.

Zerche, L. 1991: Beitrag zur Taxonomie und Verbreitung der Gattung Deliphrosoma Reitter, 1909 (Coleoptera, Staphylinidae, Omaliinae). - Beiträge zur Entomologie, Berlin 41 (2): 313-332. - https:// www.contributions-to-entomology.org/article/ view/1333/1332. 